\title{
Modelling the properties of magnetic clusters with complex structures: how symmetry can help us
}

\author{
Boris Tsukerblat (10 a , Andrew Palii ${ }^{\mathrm{b}, \mathrm{c}}$, Juan Modesto Clemente-Juan (D) ${ }^{\mathrm{d}}$ and \\ Eugenio Coronado (1) $d$
}

${ }^{a}$ Department of Chemistry, Ben-Gurion University of the Negev, Beer-Sheva, Israel; ${ }^{b}$ Institute of Problems of Chemical Physics, Chernogolovka, Russia; 'Institute of Applied Physics, Academy of Sciences of Moldova, Chisinau, Moldova; ${ }^{\mathrm{d}}$ Instituto de Ciencia Molecular, Universidad de Valencia Paterna, Spain

In memory of Professor Peter Day.

\begin{abstract}
The purpose of this article is to answer the question of how symmetry helps us to investigate and understand the properties of nanoscopic magnetic clusters with complex structures. The systems of choice will be the three types of polyoxometalates (POMs): (1) POMs containing localised spins; (2) reduced mixed-valence (MV) POMs; (3) partially delocalised POMs in which localised and delocalised subunits coexist and interact. The theoretical tools based on various kinds of symmetry are the following: (1) irreducible tensor operator (ITO) approach based on the so-called "spin-symmetry" and MAGPACK program; (2) group-theoretical assignment of the exchange multiplets based on spin- and point symmetries; (3) group-theoretical classification of the delocalised electronic and electron-vibrational states of MV POMs; (4) general approach (based on spin symmetry) to evaluate the energy levels of large MV clusters and the corresponding MVPACK program; (5) computational approach (employing point symmetry) to solve multidimensional non-adiabatic vibronic problems in the nanoscopic systems realized as VIBPACK software. We made it our goal to avoid a conventional deductive style of presentation. On the contrary, we first consider specially selected complex POMs and then show by what methods and in what way the theoretical problems arising in the description of the properties of these molecules can be properly solved.
\end{abstract}

\section{Contents}

\section{Introduction}

2. Magnetic polyoxometalates with localised spins

2.1. Cluster anion $\left[\mathrm{V}_{15}^{\mathrm{IV}} \mathrm{As}_{6} \mathrm{O}_{42}\left(\mathrm{H}_{2} \mathrm{O}\right)\right]^{6-}$

2.1.2. Spin-symmetry: irreducible tensor operators approach

CONTACT Boris Tsukerblat tsuker@bgu.ac.il Department of Chemistry, Ben-Gurion University of the Negev, Beer-Sheva 84105, Israel; Juan Modesto Clemente-Juan juan.m.clemente@uv.es 8 Instituto de Ciencia Molecular, Universidad de Valencia, Paterna 46980, Spain 
2.1.3. Spin-symmetry: isotropic exchange in $\mathrm{V}_{15}$ cluster and energy pattern

2.1.4. Point symmetry: group-theoretical assignation of the low-lying levels of $\mathrm{V}_{15}$

2.1.5. Anisotropic exchange interactions in $\mathrm{V}_{15}$ : a general outlook from the symmetry, pseudo-angular momentum representation

2.1.6. Magnetic anisotropy of $\mathrm{V}_{15}$ : antisymmetric exchange and stepwise magnetisation

2.2. Two hexanuclear polyoxotungstates:

$\left(n-\mathrm{BuNH}_{3}\right)_{12}\left[(\mathrm{CuCl})_{6}\left(\mathrm{AsW}_{9} \mathrm{O}_{33}\right)_{2}\right] \cdot 6 \mathrm{H}_{2} \mathrm{O}\left(\mathrm{Cu}_{6}\right)$ and $\left(n-\mathrm{BuNH}_{3}\right)_{12}$ $\left[(\mathrm{MnCl})_{6}\left(\mathrm{SbW}_{9} \mathrm{O}_{33}\right)_{2}\right] \cdot 6 \mathrm{H}_{2} \mathrm{O}\left(\mathrm{Mn}_{6}\right)$

2.2.1. Structural features of spin rings $\left[(\mathrm{CuCl})_{6}-\left(\mathrm{AsW}_{9} \mathrm{O}_{33}\right)_{2}\right]^{12-}$ and $\left[(\mathrm{MnCl})_{6}\left(\mathrm{SbW}_{9} \mathrm{O}_{33}\right)_{2}\right]^{12-}$

2.2.2. Analysis of the exchange interactions in polyoxometalates $\mathrm{Cu}_{6}$ and $\mathrm{Mn}_{6}$, magnetic anisotropy

2.3. The clusters $\left\{\mathrm{Mo}_{57} \mathrm{M}_{6}\right\}\left(\mathrm{M}=\mathrm{V}^{\mathrm{IV}}\right.$ or $\left.\mathrm{M}=\mathrm{Fe}^{\mathrm{III}}\right)$ : antisymmetric exchange in a biprismatic $\mathrm{V}_{6}$ cluster

2.3.1. Structure and magnetic sites in $\left\{\mathrm{Mo}_{57} \mathrm{M}_{6}\right\}$

2.3.2. Exchange interactions in $\left\{\mathrm{Mo}_{57} \mathrm{M}_{6}\right\}$ and magnetic anisotropy

3. Mixed-valence magnetic polyoxometalates

3.1. Reduced mixed valence polyoxovanadates $\left[\mathrm{V}_{18} \mathrm{O}_{48}\right]^{n-}(n=4 \div 18)$

3.1.1. General approach to the electronic problem of mixed valency and double exchange: exploration of spin symmetry

3.1.2. Magnetic properties of $\left[\mathrm{V}_{18} \mathrm{O}_{48}\right]^{n-}$ cluster

3.2. Mixed-valence polyoxometalates featuring delocalisation of an electronic pair: Keggin and Wells-Dawson anions reduced by two electrons

3.2.1. Structures of the Keggin and Wells-Dawson anions

3.2.2. Site symmetry approach: delocalised electronic pair in the 2e-reduced Keggin anion

3.2.3. Vibronic problem in 2e-reduced Keggin anion: formulation

3.2.4. Symmetry assisted approach to the multidimensional non-adiabatic Jahn-Teller problem

4. Mixed-valence reduced polyoxometalates with partial electron delocalisation 252

4.1. The polyoxovanadate anion $\left[\mathrm{KV}_{4}^{\mathrm{IV}} \mathrm{V}_{8}^{\mathrm{V}}\left(\mathrm{O}_{3} \mathrm{AsOH}\right)_{3} \mathrm{O}_{27}\left(\mathrm{AsO}_{4}\right)\right]^{6-}$

4.1.1. Structure of polyoxovanadate anion $\left[\mathrm{KV}_{4}^{\mathrm{IV}} \mathrm{V}_{8}^{\mathrm{V}}\left(\mathrm{O}_{3} \mathrm{AsOH}\right)_{3} \mathrm{O}_{27}\left(\mathrm{AsO}_{4}\right)\right]^{6-}$

4.1.2. Group-theoretical classification of the delocalised states in mixed-valence systems

4.1.3. Group-theoretical classification of spin multiplets in a partially delocalised system, the main features of $\mathrm{V}_{4}^{\mathrm{IV}} \mathrm{V}_{8}^{\mathrm{V}}$

4.2. Partially delocalised cluster anion $\left[\mathrm{V}_{8}^{\mathrm{IV}} \mathrm{V}_{4}^{\mathrm{V}} \mathrm{As}_{8} \mathrm{O}_{40}\left(\mathrm{H}_{2} \mathrm{O}\right)\right] 4-\equiv\left\{\mathrm{V}_{12} \mathrm{As}_{8}\right\}$ 
Funding 260

ORCID 260

References $\quad 260$

Abbreviations recurrently used in the text

$\begin{array}{ll}\text { POM } & \text { polyoxometalates } \\ \text { MV } & \text { mixed-valence } \\ \text { JT effect } & \text { Jahn-Teller effect } \\ \text { ITO } & \text { irreducible tensor operator } \\ \text { Irrep } & \text { irreducible representation } \\ \text { HDVV Hamiltonian } & \text { Heisenberg-Dirac-Van Vleck Hamiltonian } \\ \text { AS exchange } & \text { antisymmetric exchange } \\ \text { MAGPACK } & \text { program to solve eigen-problem for spin-localised systems } \\ \text { MVPACK } & \text { program to solve eigen-problem for mixed-valence systems } \\ \text { VIBPACK } & \text { program to solve the vibronic eigen-problem }\end{array}$

\section{Introduction}

Contemporary molecular magnetism represents an interdisciplinary field of science that incorporates basic concepts of physics, chemistry and material sciences [1-10]. Magnetic clusters occupy a central place in this field owing to the possibility of serving as model systems to understand in detail the electronic processes controlling their properties, namely the magnetic exchange, the magnetic anisotropy and the electron delocalisation. This knowledge has been crucial to develop an extensive family of magnetic molecules, known as single-molecule magnets, exhibiting slow magnetic relaxation of the magnetisation at low temperatures, together with marked quantum effects. A versatile series of compounds in which these magnetic clusters can be isolated are the so-called polyoxometalates (POMs). These coordination compounds provide an ideal platform to study these electronic processes at the molecular level. Thus, thanks to the rigidity imposed by the molecular metal oxide frameworks acting as ligands, these magnetic POMs often exhibit high symmetries. In addition, these compounds can accept a defined number of delocalised electrons in their structures (mixed-valence POMs), having at the same time the ability to accommodate at specific positions paramagnetic metal ions. The exploitation of these magnetic and electronic functionalities has been the subject of extensive research in the last 20 years, [11], which complements other more traditional applications of these anionic metal oxides such as catalysis or medicine [12].

Recently, the interest for these clusters has evolved from their use as model systems in molecular magnetism, to the use of these clusters in molecular spintronics and quantum computing [13]. In molecular spintronics the mixed-valence (MV) POMs are especially promising when along with localised spins they host also itinerant electrons. Such a kind of reduced magnetic POMs are advantageous owing to the possibility to accept various numbers of delocalised electrons and able to mediate spin coupling between the localised spins. Since the itinerant electrons are quite sensitive to the external stimuli due to their mobility, one can hope to efficiently control the spin states of these systems by applying, 
for example, an external electric field [14-17]. In quantum computing, magnetic POMs have also shown to provide unique examples of highly coherent spin qubits and the possibility to create molecular spin quantum gates that are able to perform quantum algorithms [13,18-29].

Studies of magnetic clusters started from small relatively simple, mainly symmetric, systems such as dimeric, trimeric and tetrameric clusters for which the energy levels within the model of isotropic magnetic exchange can be found in an analytical way using well known spin coupling expressions or Kambe's method. Further development of molecular magnetism led to evolution towards analysis of more complex nanoscopic systems and more complex anisotropic forms of the exchange interactions in accordance with the needs of numerous applications such as molecular biology, nanomagnetism and spintronics. The understanding of the magnetic and spectroscopic properties of these nanoscopic molecular systems have required special theoretical concepts well adapted to accurate description of their electronic, magnetic and vibronic structures. In this respect the symmetry of these objects plays crucial role for the following reasons: (1) as distinguished from the numerical methods, the symmetry based consideration does not use approximations and particular approximate models and in this sense the results derived in this way can be regarded as 'exact' ones; (2) application of the symmetry arguments allows us to simplify the numerical evaluation to the maximum possible extent. This is especially important for modelling the properties of nanoscopic POMs (and for the large molecular systems, in general), which are often inaccessible even using contemporary computational resources; (3) in many cases, especially when the molecular symmetry is high enough, the use of symmetry arguments facilitate qualitative understanding of the physical phenomena without appeal to a particular physical model. It is worth noting in this context that symmetry underlays the key laws of the nature, such as conservation laws; (4) finally, the symmetry laws impose restrictions on possible results obtained in different approximate models (which sometimes is hardly to rigorously justify) and this seems to be especially useful in the rigorous analysis of such complex systems.

The main aim of this article is to present the theoretical tools of Molecular Magnetism based on the symmetry concepts. We made it our goal to avoid a conventional deductive style of presentation within which the computational methods are described and then accompanied by examples of applications. On the contrary, we first consider specially selected POM molecules with a complex structure, and then show by what methods and in what way the theoretical problems arising in the description of the properties of these molecules can be properly solved. In this regard, the three basic types of the magnetic POMs will be discussed: (1) POMs containing localised spins carried by the metal ions with unfilled shells (cluster anion present in $\mathrm{K}_{6}\left[\mathrm{~V}_{15}^{\mathrm{IV}} \mathrm{As}_{6} \mathrm{O}_{42}\left(\mathrm{H}_{2} \mathrm{O}\right)\right] \cdot 8 \mathrm{H}_{2} \mathrm{O}$ conventionally referred to as $\mathrm{V}_{15}$, the hexa-nuclear spin rings in the two polyoxotungstates, $\left(n-\mathrm{BuNH}_{3}\right)_{12}\left[(\mathrm{CuCl})_{6}-\left(\mathrm{AsW}_{9} \mathrm{O}_{33}\right) 2\right] \cdot 6 \mathrm{H}_{2} \mathrm{O}$ and $(n-$ $\left.\mathrm{BuNH}_{3}\right)_{12}\left[(\mathrm{MnCl})_{6}\left(\mathrm{SbW}_{9} \mathrm{O}_{33}\right)_{2}\right] \cdot 6 \mathrm{H}_{2} \mathrm{O},\left\{\mathrm{Mo}_{57} \mathrm{M}_{6}\right\}$ cluster $)$; (2) MV reduced POMs containing metal ions in different oxidation degrees whose electrons are delocalised among a definite number of magnetic metal sites (polyoxovanadates $\left[\mathrm{V}_{18} \mathrm{O}_{48}\right]^{n-}(n=4 \div 18)$, reduced Keggin and Wells-Dawson anions) and, finally (3) partially delocalised POMs in which localised and delocalised magnetic subunits coexist and interact (polyoxovanadate $\left[\mathrm{KV}_{4}^{\mathrm{IV}} \mathrm{V}_{8}^{\mathrm{V}}\left(\mathrm{O}_{3} \mathrm{AsOH}\right)_{3} \mathrm{O}_{27}\left(\mathrm{AsO}_{4}\right)\right]^{6-}$, cluster anion $\left.\left[\mathrm{V}_{8}^{\mathrm{IV}} \mathrm{V}_{4}^{\mathrm{V}} \mathrm{As}_{8} \mathrm{O}_{40}\left(\mathrm{H}_{2} \mathrm{O}\right)\right]^{4-}\right)$. 
In connection with the proper description of the properties of mentioned classes of POMs the following main theoretical models based on various kinds of symmetry tools will be discussed: (1) approach based on the ITO of the full rotation group that is based on the so-called 'spin-symmetry' [30-40]. This ITO approach accompanied by the powerful MAGPACK program [41] as applied to the molecular magnetism allows the evaluation of spin levels, thermodynamic and spectroscopic properties of mesoscopic metal clusters in the framework of the isotropic and anisotropic spin-Hamiltonians; moreover, any interactions expressed through the angular momenta operators of arbitrary nature can be incorporated into the computational scheme; (2) group-theoretical assignment of the exchange multiplets based on spin- and point symmetries that allows to analyse the anisotropic forms of the exchange interaction and magnetic anisotropy in general terms, to derive selection rules for magnetic resonance transitions, to reduce the eigen-problem and to reveal the active vibrational Jahn-Teller modes in case of degenerate electronic terms [34,38,39]; (3) group-theoretical classification of the delocalised electronic and electron-vibrational states of MV POMs that essentially reduces the time of calculations and provides access to the selection rules for different kinds of transitions [34]; (4) general (based on spin symmetry) approach to evaluate the energy levels of large MV magnetic POMs in combination with the corresponding MVPACK program [42]; (5) computational approach (based on point symmetry) to solve multidimensional non-adiabatic (dynamic) vibronic problems in the nanoscopic systems realised as the VIBPACK software [43]. The proposed approach is based on the full exploration of the point symmetry of the electronic and vibrational states and can enormously extend computational resources in the dynamical problem of mixedvalency in reduced magnetic POMs. The programs MAGPACK, MVPACK and VIBPACK constitute the full set of the tools which cover the theoretical background of Molecular Magnetism (for systems with a relatively weak spin-orbit coupling) in the framework of parametric (semiempiric) approach.

\section{Magnetic polyoxometalates with localised spins}

\subsection{Cluster anion $\left[\mathrm{V}_{15}^{\mathrm{IV}} \mathrm{As}_{6} \mathrm{O}_{42}\left(\mathrm{H}_{2} \mathrm{O}\right)\right]^{6-}$}

\subsubsection{Structural peculiarities of $V_{15}$ cluster anion}

This cluster anion, conventionally referred to as $\mathrm{V}_{15}$, is present in $\mathrm{K}_{6}\left[\mathrm{~V}_{15}^{\mathrm{IV}} \mathrm{As}_{6} \mathrm{O}_{42}\left(\mathrm{H}_{2} \mathrm{O}\right)\right] \times$ $8 \mathrm{H}_{2} \mathrm{O}$ and involves fifteen $\mathrm{V}^{\mathrm{IV}}$ ions carrying localised spins $S_{i}=1 / 2$. These $\mathrm{V}^{\mathrm{IV}}$ ions form a large central triangle which is sandwiched by two distorted hexagons $[9 b, 44]$ so that the overall symmetry is $\mathbf{D}_{3}$ (Figure 1 ). The layered structure of the molecule is given in Figure 1a, while the skeleton formed by the vanadium ions is presented in Figure 1b. In the following discussion we will also appeal to the classical spin frustrated arrangement depicted in Figure 1c.

This system was discovered almost three decades ago [9b,44] but still continues to attract attention as molecular magnet with unique structure and properties, and also as an exceptional object for the study of the antisymmetric (AS) exchange interaction [45]. Analysis of the magnetic and spectroscopic properties of this fascinating system constitutes a distinct trend in the study of complex nanoscopic polyoxometalates. Studies of the adiabatic magnetisation and quantum dynamics of the $\mathrm{V}_{15}$ cluster with $S=1 / 2$ ground state showed that this system exhibits a hysteresis magnetisation loop of pure molecular origin and thus can 

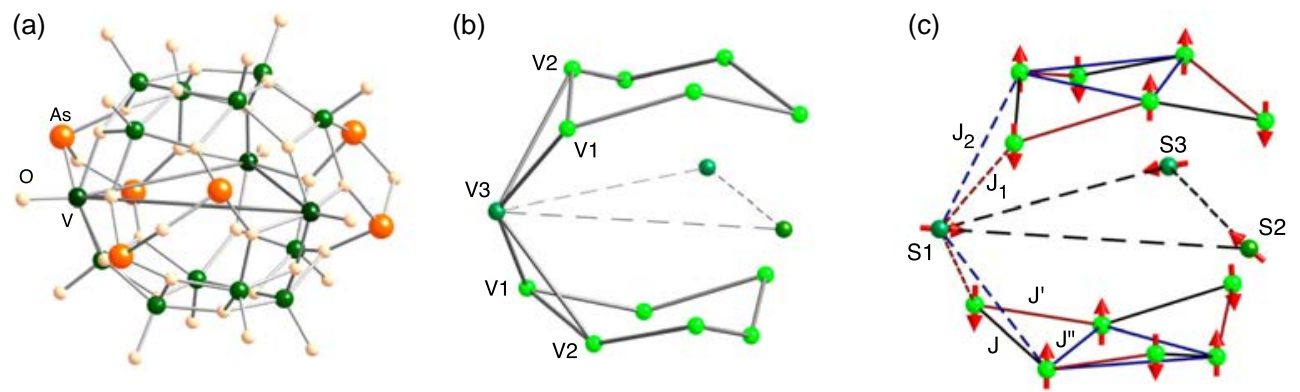

Figure 1. Structure of the polyoxovanadate $\mathrm{K}_{6}\left[\mathrm{~V}_{15}^{\mathrm{IV}} \mathrm{As}_{6} \mathrm{O}_{42}\left(\mathrm{H}_{2} \mathrm{O}\right)\right] \cdot 8 \mathrm{H}_{2} \mathrm{O}$. Scheme of the V15 metal network (green balls) (a); scheme of the vanadium network with the indication of three different vanadium (V1, V2 and V3) positions and frustrated spins (S1, S2, S3) of the central triangle (b); dominant exchange pathways, pictorial representation of classical spin arrangement illustrating strongly coupled spins in the external hexagons in the ground state and frustration effect in the central triangle expressed as non-colinear spin arrangement (c). Colour online.

be referred to as a mesoscopic system [45]. On the other hand, frozen solutions of this cluster exhibited at low temperature Rabi oscillations, which were indicative of large quantum coherence times $\mathrm{T}_{2}$. Thus, it constituted the first example of molecular magnet behaving as a spin qubit (in view of long coherence time) [46] due to which the $\mathrm{V}_{15}$ cluster proved to be a prerequisite for the use of molecular magnets in quantum computing.

\subsubsection{Spin-symmetry: irreducible tensor operators approach}

In the transition metal clusters with localised spins and orbitally non-degenerate ground terms of the constituent ions, the leading contribution to the exchange interaction is usually represented by the isotropic Heisenberg-Dirac-Van-Vleck (HDVV) spin-Hamiltonian:

$$
H_{0}=-2 \sum_{i<j} J_{i j} S_{i} \cdot S_{j}
$$

In Equation (1) $S_{i}$ are the vector spin operators of the full electronic shells of the constituent ions, the values $J_{i j}$ are the exchange parameters, and the summation is extended over all pairwise interactions. The HDVV Hamiltonian operates in the space of spinfunctions associated with the spins $S_{1}, S_{2}, \ldots S_{N}$ ( $N$ is the full number of the magnetic ions) so that the dimension of the Hilbert space is determined by the product $\left(2 S_{1}+1\right) \times\left(2 S_{2}+1\right) \ldots \times\left(2 S_{N}+1\right)$. In some cases of the systems with symmetric geometry and restricted nuclearity, such as dimers, symmetric trimers (see Equations (4) and (5)), tetramers one can find an analytical solution of the exchange problem that means to express the energy levels as functions of the total spin of the system and intermediate spins in an appropriate spin coupling scheme (see more details in Ref. [38]). More sophisticated approaches are developed in the two articles $[37 \mathrm{~b}, \mathrm{c}]$ in which the exact solution for the energy pattern of the finite spin-chains has been found. But, in general, the problem requires numerical diagonalization of the HDVV Hamiltonian in the basis corresponding to full Hilbert space. For the polyatomic systems the full Hilbert space is rather large, especially in the case of large constituent spins. The solution of a rather complicated eigenproblem and rationalisation of the results can be obtained by the use of various symmetry 
tools. In these cases, the problem can be essentially simplified by the use of the so-called 'spin-symmetry' which can be employed through the ITO approach. Actually, the ITOs belong to the spherical group of symmetry (or more general unitary groups that is beyond of the topic of present article).

The ITO approach allows to evaluate the matrix elements of the exchange Hamiltonian of the most general form which includes both isotropic and anisotropic parts. All relevant interactions, such as single-ion anisotropy, Zeeman and Stark interactions can be included in the general scheme of calculation. Application of the ITO approach is especially efficient when the isotropic part is the leading contribution to the exchange coupling. The approach is formulated for the system with an arbitrary number of magnetic sites, $N$, having local spins $S_{1}, S_{2}, \ldots, S_{N}$. It is assumed that the spin 2 is coupled to spin 1 to give spin $S_{12}$, that can be expressed as $S_{1}+S_{2}=S_{12}$. Then the next spin 3 is added so that we obtain $S_{12}+S_{3}=S_{123}$. It is convenient to employ this successive spin vector coupling scheme

$$
S_{1}+S_{2}=\tilde{S}_{2}, \tilde{S}_{2}+S_{3}=\tilde{S}_{3}, \ldots \tilde{S}_{N-1}+S_{N}=S
$$

where $\tilde{S}_{2}=S_{12}, \tilde{S}_{3}=S_{123}$, etc. are the intermediate spins in the adopted spin coupling scheme and $S$ is the total spin of the system. The choice of the numbers of spins in this scheme is arbitrary and is not interrelated with special positions of the spin carriers in the magnetic molecule. Therefore, spin functions of the system can be labelled as

$$
\left|S_{1} S_{2}\left(\tilde{S}_{2}\right) S_{3}\left(\tilde{S}_{3}\right) \ldots S_{N-1}\left(\tilde{S}_{N-1}\right) S_{N} S M \equiv\right|(\tilde{S}) S M
$$

where $(\tilde{S})$ symbolises the set of the intermediate spin quantum numbers as indicated in Equation (3). The direct way to find the energy levels of the system described by the HDVV Hamiltonian, Equation (1), is to build the many-particle spin-functions (or alternatively, to work in terms of Slater determinants) and to evaluate the matrix of the Hamiltonian. However, this way is usually almost impracticable even for relatively small systems, such as trimeric and tetrameric exchange clusters, especially when the local spins exceed $1 / 2$. It is important to emphasise the crucial advantage of the ITO approach as a computational tool. The ITO approach does allow to avoid evaluation of the many-particle spin functions (or alternatively, appropriate combinations of Slater determinants) and to directly find the matrix elements of all kinds of isotropic and anisotropic exchange interactions using only the symbolic representation in which spin coupling scheme is specified, like in Equation (3). These matrix elements of the full exchange Hamiltonian link the states with different quantum numbers introduced in the definition of the spin-functions, Equation (3). Actually, symbolic representation means that the mentioned matrix elements are expressed in terms of all intermediate and total spins of the involved states, while the exchange parameters remain as parameters to be determined additionally. Therefore, the procedure does not suppose neither evaluation of the many-spin functions nor any operations with the Slater determinants.

We omit here the mathematical details of calculations and final expressions referring to the applications of ITO in molecular magnetism which are reviewed in full details in Refs. $[2,3,34,35,36,37,38,39]$. From the practical point of view, it is convenient to use the MAGPACK software package [41] fully based on the use of ITO technique. This software allows to solve the eigen-problem for large spin clusters taking into account all isotropic and anisotropic interactions and to derive their thermodynamic and spectroscopic (mainly, inelastic neutron scattering) properties. 
A specially important case is represented by the isotropic HDVV exchange for which the full Hilbert space can be reduced to get block-structure of the full Hamiltonian matrix according to total spin values $S$. The HDVV Hamiltonian commutes with the operator $S^{2}$, so that total spin $S$ of the system is always a good quantum number (but not the intermediate spins!). It is important to note that only the spin functions with the same values of $S$ (and its projections $M_{S}$ ) are mixed, as prescribed by the general rule of symmetry (since they belong to the same irreducible representations $D^{(S)}$ of the rotation group). Using this rule, one can state that the solution of the HDVV eigen-problem can be reduced to the evaluation of the matrix elements connecting the states having equal total spins $S$. It is to be noted that the MAGPACK software is the most advantageous tool for treating the bilinear isotropic HDVV exchange. The presence of the anisotropic terms reduces the symmetry of the Hamiltonian and therefore the states with different $S$ prove to be mixed. This leads to the increase of the sizes of the matrices to be diagonalised. Thus, in the most unfavourable case of the fully anisotropic Hamiltonian (when the spin-symmetry is lost) the advantages of the ITO approach are reduced so that the basis consisting of simple direct products of spin-functions can be used and, moreover, can provide computational advantages.

In recent years, the computational tools for the needs of contemporary chemistry have been replenished with powerful new computer programs with a wide range of applications, including accurate analysis of magnetic resonance parameters. In this respect one should mention PHI, a computer program designed for the calculation of the magnetic properties of paramagnetic coordination complexes [47a]. Then, MAGELLAN is a computer program [47b] designed for the determination of the orientation of the magnetic anisotropy of the $m_{\mathrm{J}}= \pm^{15} / 2$ state of Dy ${ }^{\mathrm{III}}$ via electrostatic optimisation of the aspherical electron density distribution.

Concluding this section it is worth to specially mention that not only magnetic interactions expressed through spin-operators associated with electronic spins (such as isotropic exchange, Equation (1)) can be incorporated in the computational approaches based on ITO. In fact, the angular momenta operators of any nature, such orbital angular momentum (vector $\boldsymbol{L}$ with components $\left.L_{x}, L_{y}, L_{z}\right)$, total angular momentum $(\boldsymbol{J})$, nuclear spin operator $(I)$ can be expressed in terms ITO. Therefore, the corresponding interactions, such as spin-orbital coupling, isotropic and anisotropic Zeeman and hyperfine electron-nuclear interactions can be treated using ITO technique. In particular, the hyperfine interactions with nuclear spins (at least with those located at the metal ions), which can lead to very important effects and largely determine spin dynamical phenomena, can be incorporated into the same formalism.

\subsubsection{Spin-symmetry: isotropic exchange in $V_{15}$ cluster and energy pattern}

Let us illustrate application of the ITO approach by considering the $V_{15}$ cluster. For fifteen spins $1 / 2$ the total spin of the system varies from $S=1 / 2$ to $S=15 / 2$, wherein each total spin state arises from different combinations of intermediate spins and therefore is repeated many times. The dimension of the matrix of the exchange Hamiltonian involves $2^{15}=32768$ states and thus, it is rather large. The use of spin-symmetry essentially reduces the dimensions of energy matrix as shown in Table 1 indicating sizes of the blocks corresponding to each total spin value $S$, i.e. the numbers of the levels with certain values of $S$. The largest size (2002) has the submatrix for the total spin $S=3 / 2$ levels (indicated in bold in Table 1). The set of the isotropic exchange parameters $J^{*}, J_{1}, J^{\prime}, J_{2}, J^{\prime \prime}$ 
Table 1. Dimensions of the submatrices for the $V_{15}$ system in the model of the isotropic exchange.

\begin{tabular}{lcccccccc}
\hline Total spin S & $1 / 2$ & $\mathbf{3} / \mathbf{2}$ & $5 / 2$ & $7 / 2$ & $9 / 2$ & $11 / 2$ & $13 / 2$ & $15 / 2$ \\
\hline Block size & 1430 & $\mathbf{2 0 0 2}$ & 1638 & 910 & 350 & 90 & 14 & 1 \\
\hline
\end{tabular}

Notes: The block of the highest dimension is highlighted by bold.

(Figure 1c) have been found from the temperature dependence of the magnetic susceptibility $\left(J^{*}=-170 \mathrm{~cm}^{-1}, J_{1}=J^{\prime}=-28 \mathrm{~cm}^{-1}, J_{2}=J^{\prime \prime}=-56 \mathrm{~cm}^{-1}\right.$ [45]. The energy pattern of $\mathrm{V}_{15}$ numerically evaluated with the help of MAGPACK and the parameters so far mentioned is shown in Figure 2. The composition of the levels is rather complicated due to very high density of spin levels arising from the numerous exchange pathways and large spin space. Nevertheless, some important peculiarities can be deduced at a glance. Thus, the energy pattern shows that the ground state is the antiferromagnetic state having the total spin $S=1 / 2$ and the first excited state with $S=3 / 2$ proves to be very close to the ground state. The energy gap separating the two levels from the $S=3 / 2$ is approximately $2.5 \mathrm{~cm}^{-1}$ and so these two low-lying levels are well isolated from the higher states that lie more than $500 \mathrm{~cm}^{-1}$ above. These highly excited states are related mostly to the excitations in strongly paired spins in the external hexagons. It is to be underlined that the ground state comprises two $S=1 / 2$ doublets resulting in a four-fold degenerate level) and therefore, the system exhibits additional degeneracy. This accidental degeneracy is in some sense a surprising observation as it is not related to a requirement of the point group symmetry $\mathbf{D}_{3}$ of the molecule.

This result shows that the low-lying levels can be viewed as the energy pattern of an isolated spin-1/2 triangle (Figure 1a) in which the effective coupling between the $\mathrm{V}$ ions, transmitted via the spin-paired hexagons as shown in Figure 1b, is relatively small. To clarify this point let us consider the exchange interaction in a symmetric trinuclear system which can be described by the following HDVV Hamiltonian:

$$
H_{0}=-2 J_{0}\left(S_{1} S_{2}+S_{2} S_{3}+S_{3} S_{1}\right)
$$

where $S_{1}, S_{2}$ and $S_{3}$ denote the spin operators on the sites 1, 2 and 3. The following spin coupling scheme for three spins, $S_{1} S_{2}\left(S_{12}\right) S_{3} S \equiv\left(S_{12}\right) S$ is assumed with $S_{12}$ being the intermediate spin $\left(S_{12}=S_{1}+S_{2}\right)$. The energy levels $\varepsilon_{0}(S)$ are expressed as

$$
\varepsilon_{0}(S)=-J_{0}\left[S(S+1)-\sum_{i=1}^{3} S_{i}\left(S_{i}+1\right)\right]
$$

It is seen that they do depend upon the total spin $S$ but they are independent of $S_{12}$. This leads to the 'accidental' four-fold degeneracy of the two $S=1 / 2$ doublets in the energy pattern of a triangular unit of $\mathrm{V}_{15}$. This degeneracy, which can be referred to as 'accidental' (at this stage of consideration) within the HDVV model, has important consequences which will be discussed later on. The model of an effective spin triangle for the low-lying spin excitations was proposed in Ref. [9b] and substantiated within the perturbation theory. As we will see below this degeneracy is a consequence of the point symmetry, which will be specially discussed below with regards to the magnetic anisotropy of $\mathrm{V}_{15}$. 


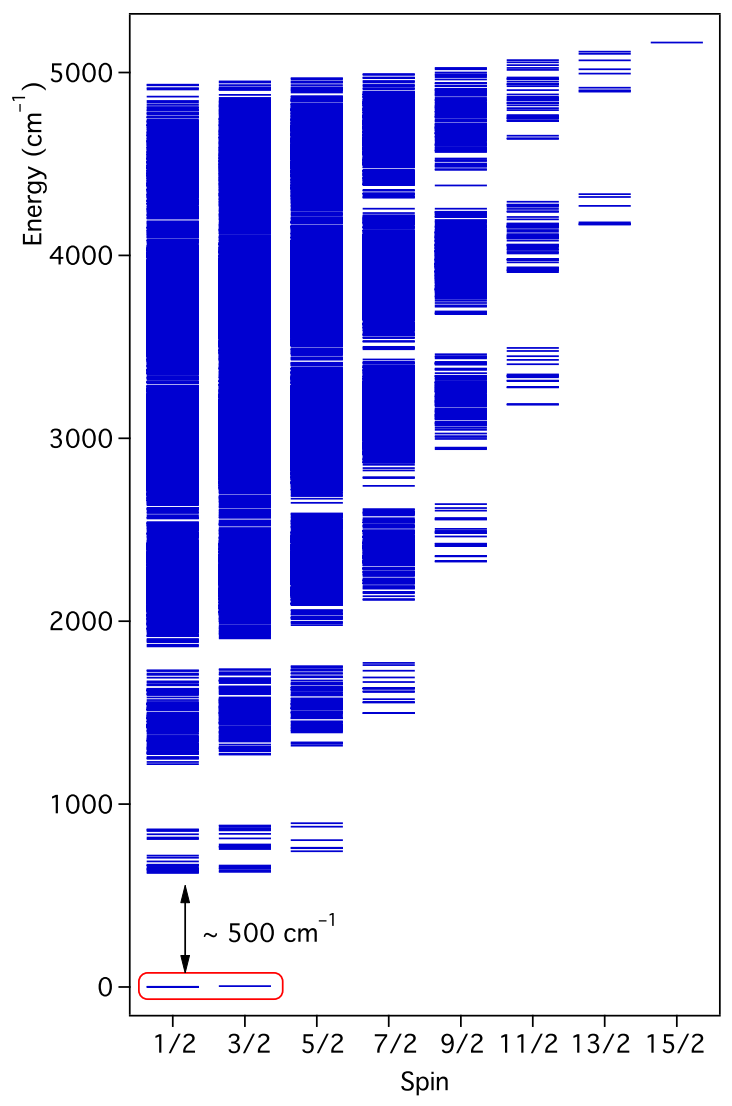

Figure 2. Energy pattern of the $\mathrm{V}^{15}$ system evaluated in the HDVV model. The energies are arranged in columns according to the total spin of the system. The two low-lying levels comprising two $S=1 / 2$ doublets (ground) and a spin quadruplet $S=3 / 2$ (excited) are circled.

\subsubsection{Point symmetry: group-theoretical assignation of the low-lying levels of $V_{15}$}

A concept of the group-theoretical classification (assignment) of the exchange multiplets appeared as a tool to elucidate the nature of so-called 'accidental' degeneracies of the spin states in symmetric exchange clusters $[34,38,39]$. Let us consider a trigonal trimer with one unpaired electron per site $\left(d^{1}-d^{1}-d^{1}\right)$ in more detail, keeping in mind the lowlying spin excitations in $V_{15}$. As we could see, the degeneracy of the two spin doublet states exceeds that allowed by the trigonal point symmetry and, therefore, the theoretical model requires additional careful examination. Let us note an obvious fact that HDVV Hamiltonian operates in the spin-space and, therefore, the energy levels are labelled by the spin quantum numbers only, while each eigenstate of the many-electron first principle Hamiltonian (terms in spectroscopic notations) must be labelled by the total spin as well as by an irreducible representation (irrep) of the point group. Therefore, the question appears of how the spin states in HDVV model are interrelated to the exact terms of the system. The answer is provided by the group-theoretical classification procedure $[34,38,39]$, which makes possible to establish one-to-one correspondence between the states $D^{(S)}$ (irreps of the rotation group, or, simply, the set of states with a given $S$ ) obtained in the HDVV model 
and the $S \Gamma$ - terms of the system, where $\Gamma$ is the irreducible representation of the point group.

The symbolic expression of the coupling scheme for three spins $S_{i}=1 / 2$ in terms of the irreps of the rotation group, $D^{(S)}$, is as following:

$$
D^{(1 / 2)} \otimes D^{(1 / 2)} \otimes D^{(1 / 2)}=2 D^{(1 / 2)}+D^{(3 / 2)} .
$$

Here the symbol $\otimes$ means the direct product of the basis functions (that means the set of functions belonging to the product of all $S_{1}$ and $S_{2}$ states) and $2 D^{(1 / 2)}$ means the presence of the two $S=1 / 2$ states. The two $S=1 / 2$ levels $\left(2 D^{(1 / 2)}\right)$ arises from the two intermediate spins $S_{12}=0$ and $S_{12}=1$ giving rise to the two $S=1 / 2$ total spin states according to the rule of spin coupling. For illustration of the approach let us consider a Slater determinant $\left|a_{m_{a}}\left(S_{a}\right) b_{m_{b}}\left(S_{b}\right) c_{m_{c}}\left(S_{c}\right)\right|$ for a tri-centre system, where $a_{m_{a}}\left(S_{a}\right)$ is a spin-orbital of the ion $a$ with spin $S_{a}$ and spin projection $m_{a}$. The determinant state $\left|a_{1 / 2}\left(\frac{1}{2}\right) b_{1 / 2}\left(\frac{1}{2}\right) c_{1 / 2}\left(\frac{1}{2}\right)\right|$ with maximal total spin projection $M=m_{a}+m_{b}+m_{c}=3 / 2$ definitely belongs to the maximal total spin $S=3 / 2$. Operations of the trigonal point group induce permutations of the localised orbitals, for example

$$
\begin{aligned}
\hat{C}_{3}\left|a_{1 / 2}\left(\frac{1}{2}\right) b_{1 / 2}\left(\frac{1}{2}\right) c_{1 / 2}\left(\frac{1}{2}\right)\right| & =\left|b_{1 / 2}\left(\frac{1}{2}\right) c_{1 / 2}\left(\frac{1}{2}\right) a_{1 / 2}\left(\frac{1}{2}\right)\right| \\
& =\left|a_{1 / 2}\left(\frac{1}{2}\right) b_{1 / 2}\left(\frac{1}{2}\right) c_{1 / 2}\left(\frac{1}{2}\right)\right| .
\end{aligned}
$$

One can thus conclude that the character of the rotation $\hat{C}_{3}$ is $\chi^{(M=3 / 2)}\left(\hat{C}_{3}\right)=+1$. Applying the remaining symmetry operations to the sets of Slater determinants containing different possible spin-orbitals one can find the characters of the reducible representations spanned by these determinants. Finally, one can find the following one-to-one correspondence between the HDVV multiplets $n D^{(S)}$ ( $n$ is the number of the levels with the same spin $S$ ) and the exact terms $S \Gamma$ of the triangular system that can be symbolically expressed as

$$
2 D^{(1 / 2)} \rightarrow^{2} E, \quad D^{(3 / 2)} \rightarrow^{4} A_{2} .
$$

A detailed description of the general procedure of the group-theoretical classification based on the analysis of permutation (or unitary) symmetry groups is given in Refs. [38,39]. It is important to emphasise that this procedure does not deal with the Slater determinants and therefore allows to avoid a fairly lengthy calculations of complex combinations of determinants. The general approach allows to solve the problem for the systems of any symmetry with the arbitrary spins of the magnetic ions. The full description of the general approach requires to introduce concepts of more common unitary symmetry groups and more sophisticated group-theoretical approaches which are beyond the scope of this article.

The ITO approach so far described employs only the spin symmetry which means that the matrices of the HDVV Hamiltonian are reduced to reach block-structure according to the total spin quantum number. An essential progress in computational molecular magnetism is marked by the development of a new efficient approach which combines spin symmetry and point symmetry [48a,b]. Within this approach one can reduce the matrices of the full exchange Hamiltonian into blocks of smaller dimensions not only according 
to spin quantum number but additionally according to the irreducible representations of the point group of the system. This reduction of the dimension of the Hilbert space is especially important for the treatment of large nanoscopic spin-systems for which the traditional computational resources are insufficient. The mathematical details and physical applications of this approach can be found in Refs. [48a,b].

The problem of interrelation between the symmetry and energy spectrum of highnuclearity spin clusters has been considered in Ref. [48c]. A general and very efficient procedure developed in this work allows us to reduce the computational efforts for the calculation of the energy levels of high-nuclearity spin clusters. Using the proposed method one can perform of block factorising the Hamiltonian matrix using the invariance of the spin Hamiltonian with regard to interchanges of spin sites in any arbitrary spin Hamiltonian. In order to demonstrate the flexibility in handling different spin Hamiltonian terms and symmetry groups, its application to several types of clusters was discussed. The full description, elaborated examples and mathematical details can be found in Ref. [48c].

\subsubsection{Anisotropic exchange interactions in $V_{15}$ : a general outlook from the symmetry, pseudo-angular momentum representation}

The symbolical Equation (8) allows to draw the following conclusions about the exchange interactions in the vanadium triangle which are based only on the symmetry of the system:

(i) The 'accidental' degeneracy of the two doublets $\left(S_{12}\right) S=(0) 1 / 2,(1) 1 / 2$ is actually interrelated with the orbital degeneracy of the exact electronic states in the triangle of vanadium ions. The ground term is the orbital doublet, ${ }^{2} E$, while the excited level is the orbital singlet ${ }^{4} A_{2}$. The orbital degeneracy is inherently related to the spinfrustration in the ground state, which is schematically shown in Figure 1c. This means that the three antiferromagnetic connections in a symmetric spin triangle cannot be arranged in the classical spin picture, or alternatively, that in an antiferromagnetic triangle not all pairs of interacting spins assume their minimal pair energy in the total ground state. An interesting issue of spin-frustration has been discussed in Ref. [49]. From the quantum-mechanical point of view this situation is interrelated with the orbital degeneracy. It is to be noted that the antiferromagnetic spin connections in hexagonal moieties are saturated and not subjected to spin frustration.

(ii) As it follows from the conventional selection rules, ${ }^{2} E$ term in trigonal point groups is split by the spin-orbital interaction that acts as a first order perturbation. To reveal the symmetry of the fine structure levels one can decompose the direct product of the irreps corresponding to the two $s=1 / 2$ spin-functions (that is $D^{(1 / 2)}$ ) and orbital components $E$. This can be done with the use of the tables in Ref. [50] which contains exhaustive and instructive information about the properties of the thirtytwo point groups. Two resulting Kramers doublets can be specified as a pair of the double-valued irreps $\bar{A}_{1}+\bar{A}_{2} \equiv 2 \bar{A}$ and $\bar{E}$ of the point group [50]. In the framework of spin-Hamiltonian approach spin-orbital interaction can be described by the antisymmetric (AS) exchange:

$$
H_{A S}=\sum_{i, k} \boldsymbol{D}_{i j}\left[S_{i} \times S_{k}\right],
$$




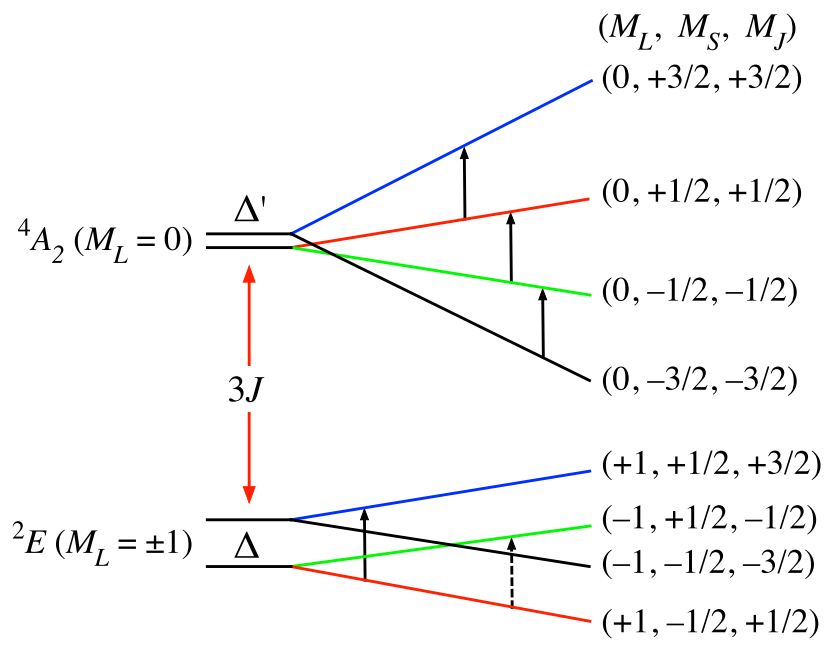

Figure 3. Energy levels of a symmetric $d^{1}-d^{1}-d^{1}$ spin triangle and the Zeeman splitting in the magnetic field $\boldsymbol{H} \| C_{3}$ axis with the indication of the quantum numbers in the pseudo-angular momentum representation.

where $\boldsymbol{D}_{i j}=-\boldsymbol{D}_{j i}$ are the antisymmetric vector parameters. AS exchange, which acts as a first order perturbation in orbital multiplets, was shown to result in a strong magnetic anisotropy and special shape of the steps in magnetisation versus field in $\mathrm{V}_{15}$ observed experimentally (see [45] and references therein). In this sense the grouptheoretical assignment allows to discuss the magnetic anisotropy in general terms based solely on the symmetry arguments without any appeal to the quantum chemical calculations.

(iii) The orbital singlet ${ }^{4} A_{2}$ (excited $S=3 / 2$ level) is split by the second order spin-orbital interaction. In the framework of the spin-Hamiltonian approach this splitting can be described by the conventional zero-field Hamiltonian

$$
H_{Z F S}=D_{0}\left[\hat{S}^{2}-(1 / 3) S(S+1)\right]
$$

in which $D_{0}$ is the zero-field splitting parameter. Figure 3 illustrates the energy pattern of $V_{15}$ cluster (obtained within three-spin model). It exhibits two spin levels with $S=$ $1 / 2$ and $S=3 / 2$ split by AS exchange in the first and second orders of perturbation theory, correspondingly.

(iv) Assignment of HDVV multiplets $n D^{(S)}$ to the exact terms $S \Gamma$ allows the usage of the so-called pseudo-angular momentum representation. In this way, one can discuss the magnetic anisotropy and to derive the selection rules in magnetic resonance transitions. This was considered in detail in Ref. [45] while discussing the experimental data on EPR in $V_{15}$ cluster. Thus, for the $S=1 / 2$ basis $|(0) 1 / 2, \pm 1 / 2\rangle,|(1) 1 / 2, \pm 1 / 2\rangle$ of the vanadium triangle in $\mathrm{V}_{15}$ the irrep $E$ can be associated with the two projections $M_{L}=+1$ and $M_{L}=-1$ of the fictitious orbital angular momentum $L=1$, and the basis functions $u_{L M_{L}}\left(S, M_{S}\right) \equiv u_{M_{L}}\left(S, M_{S}\right)$ can be found as the circular superpositions of spin-function with two intermediate spins $\left(S_{12}=0\right.$ and $\left.S_{12}=1\right)$ and total 
$\operatorname{spin} S=1 / 2[38,39]:$

$$
\begin{aligned}
& u_{ \pm 1}(1 / 2, \pm 1 / 2)=\mp 1 / \sqrt{2}(|(0) 1 / 2, \pm 1 / 2 \pm i|(1) 1 / 2, \pm 1 / 2), \\
& u_{ \pm 1}(1 / 2, \mp 1 / 2)=\mp 1 / \sqrt{2}(|(0) 1 / 2, \mp 1 / 2 \pm i|(1) 1 / 2, \mp 1 / 2) .
\end{aligned}
$$

Then, one can introduce the functions $U_{S}\left(M_{J}\right)$ belonging to the projections $M_{J}=$ $M_{L}+M_{S}$ of the full pseudo-angular momentum which correspond to the Russel-Saunders coupling scheme. The level with $S=3 / 2$ is an orbital singlet corresponding thus to $M_{L}=0$; the components are labelled as $u_{0}\left(3 / 2, M_{S}\right) \equiv U_{0}\left(M_{J}\right)$ with $M_{S}= \pm 1 / 2$ and $M_{S}= \pm 3 / 2$, so that the projections $M_{J}$ are $M_{J}= \pm 1 / 2$ and $\pm 3 / 2$. The assignment of the levels based on this concept is presented in Figure 3 that shows also the Zeeman splitting in parallel field $\mathbf{H} \| C_{3}$ and the allowed EPR transitions obeying the selection rules in pseudo-angular momentum scheme.

(v) The pseudo-angular momentum scheme allows to establish the exact rules for the crossing/anticrossing of the Zeeman levels in parallel field. In fact, the levels with different $M_{J}$ arising from HDVV multiplets $S=3 / 2$ and $S=1 / 2$ undergo to exact crossing, while the levels with the same $M_{J}$ exhibit anti-crossing. Later on we will demonstrate that this conclusion emanating exclusively from the symmetry of the system is helpful in the modelling of static and dynamic magnetisation behaviour;

(vi) Finally, the knowledge of the irreps of the point group to which the exchange multiplets belong allows one to establish the selection rules for the vibronic JT coupling. In fact, the vibrational mode of the symmetry $\Gamma_{v}$ is active if the symmetric part $[\Gamma \times \Gamma]^{\text {sym }}$ of the direct product $\Gamma \times \Gamma$ contains irrep $\Gamma_{v}[34,51]$. This is a common rule which is valid for all molecular systems, but this rule becomes actually applicable to spin clusters due to the group-theoretical assignment of the spin-multiplets. Note that the Tables [50] contains direct and symmetric products all irreps for the point groups and thus provide the practical receipt for the use group-theoretical assignment. In the case under consideration one can find the symmetric part $[E \times E]^{\text {sym }}=$ $A_{1}+E$, so that the double degenerate mode $\Gamma_{v}=E$ is active in the JT effect, while the totally symmetric vibration $A_{1}$ is irrelevant to the JT effect. This means that in the ground doublet ${ }^{2} E$ the trimeric system of $\mathrm{C}_{3 \mathrm{v}}$ symmetry (as well in all trigonal spin systems) can be subjected to the JT distortion or, in other words, to the JT instability. In the distorted configurations which are the isosceles triangles, the degeneracy is removed so that the spin frustration is eliminated. The JT coupling is shown to be competitive to the AS exchange so that the increase of the vibronic JT coupling decreases the magnetic anisotropy of the system. On the other hand, AS exchange tends to suppress the JT effect so that a strong (as compared to the JT coupling) AS exchange restores a symmetric configuration of spin sites. This is demonstrated in Ref. [45f] by the theoretical modelling of the field dependence of magnetisation that clearly shows the crucial role of the JT coupling in spin-frustrated systems. 


\subsubsection{Magnetic anisotropy of $V_{15}$ : antisymmetric exchange and stepwise magnetisation}

Let us consider the action of the AS exchange as a source of the magnetic anisotropy in more detail. The AS exchange is responsible for the zero-field splitting of the two spin doublets which can be estimated as follows:

$$
\Delta \equiv \varepsilon\left(M_{J}= \pm 3 / 2\right)-\varepsilon\left(M_{J}= \pm 1 / 2\right) \cong \sqrt{3} D_{n}-D_{\perp}^{2} / 8 J .
$$

It is important to underline the different physical roles of the two contributions to the AS exchange, namely, normal and in-plane components. The splitting of the ground level appears as a first order effect with respect to the normal (along $C_{3}$-axis) component of AS exchange (collective parameter $D_{n}$ ) and contains also a negative second order correction arising from the mixing of $\left(S_{12}\right) 1 / 2$ and (1)3/2 levels through the in-plane components $\left(D_{\perp}\right)$ of the AS exchange. For the sake of simplicity, the Zeeman interaction is assumed to be isotropic, $H_{Z}=\mu_{B} g \boldsymbol{H S}$, where $g$ is $g$-factor and $\mu_{B}$ is Borh magneton. The in-plane components part of AS exchange contributes mainly to the anti-crossing of the magnetic sublevels of $S=1 / 2$ and $S=3 / 2$ multiplets at high magnetic field giving rise to a specific angular dependence of the levels in this range of the field (Figure 4, inset 3 ).

Inspecting angular dependencies of the Zeeman levels at low field, one can see that the system is strongly anisotropic with the $C_{3}$ axis being the easy axis of magnetisation. Figure 5 a demonstrates that in the absence of the AS exchange the magnetisation $v s$ field shows two sharp steps. The first step arises from magnetisation at low field, while the second step appears due to the field induced transition from $M_{S}=-1 / 2$ to $M_{S}=-3 / 2$. Figure 5 demonstrates also how the lowfield behaviour influences the shape of the first step in the magnetisation $v$ s field dependence, while the second step is affected by the behaviour of the levels in the anti-crossing region. It is to be emphasised that the shapes of the two steps are interrelated with different components of the AS exchange. Thus, the dominating contribution to the shape of the first step is produced by the normal $D_{n}$ component, while the shape of the second step is determined by the in-plane component $D_{\perp}$. A unique possibility to experimentally discriminate the two parameters $D_{n}$ and $D_{\perp}$, which manifest themselves in different regions of the field, allows to estimate the parameters $D_{n}$ and $D_{\perp}$ with a high precision [ $45 c, d]$. One can conclude that the model of triangle for $V_{15}$, which is based on the symmetry arguments and includes AS exchange, perfectly agrees with experimental data on magnetisation in the full range of actual temperatures and magnetic field as shown in Figure 5b.

\subsection{Two hexanuclear polyoxotungstates: $\left(n-\mathrm{BuNH}_{3}\right)_{12}\left[(\mathrm{CuCl})_{6}\left(\mathrm{AsW} \mathrm{W}_{9} \mathrm{O}_{33}\right)_{2}\right] \cdot 6 \mathrm{H}_{2} \mathrm{O}$ $\left(\mathrm{Cu}_{6}\right)$ and $\left(\mathrm{n}-\mathrm{BuNH}_{3}\right)_{12}\left[(\mathrm{MnCl})_{6}\left(\mathrm{SbW}_{9} \mathrm{O}_{33}\right)_{2}\right] \cdot 6 \mathrm{H}_{2} \mathrm{O}\left(\mathrm{Mn}_{6}\right)$}

\subsubsection{Structural features of spin rings $\left[(\mathrm{CuCl})_{6}-\left(\mathrm{AsW}_{9} \mathrm{O}_{33}\right)_{2}\right]^{12-}$ and $\left[(\mathrm{MnCl})_{6}\left(\mathrm{SbW}_{9} \mathrm{O}_{33}\right)_{2}\right]^{12-}$}

The problem of Heisenberg's rings is of common interest for physics (ladder structures in the high Tc cuprates) [52,53] and molecular magnetism [54-57] for this reason has been extensively discussed (see Refs. [37b,c] and references thein). An essential progress has been achieved in the solution of the eigen-problem for a such kind of systems. Thus, using a method which combines reduction due to translational symmetry (represented by rotations in finite chains) and elimination of degenerate states from smaller subspaces, the 


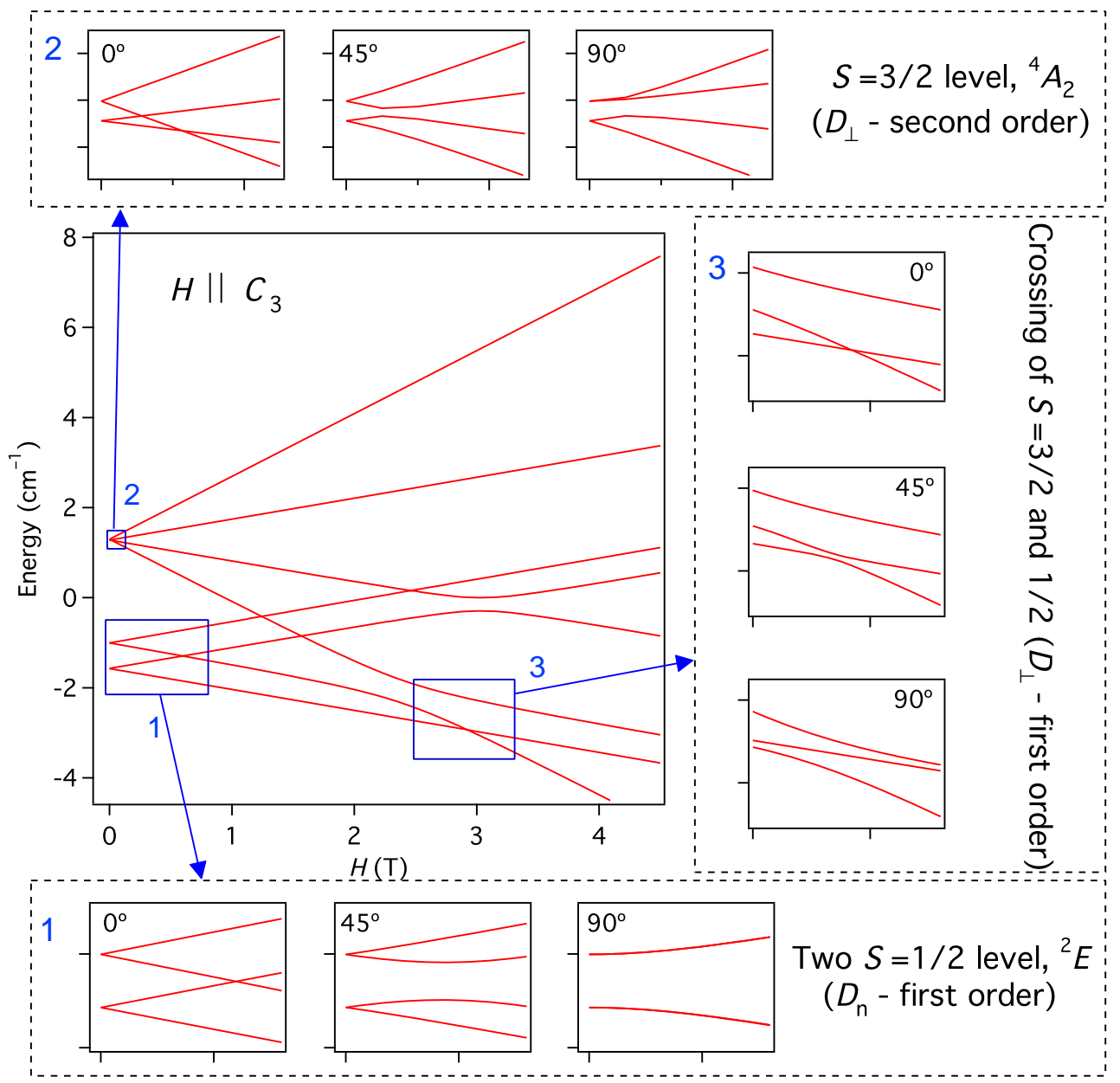

Figure 4. Zeeman levels of the vanadium $d^{1}-d^{1}-d^{1}$ spin triangle: general pattern in parallel field $\boldsymbol{H} \| C_{3}$ axis with the indication of special regions discussed in the text (central part); angular dependence of the Zeeman energy levels in low field for the two Kramers doublets (insets 1) and excited level (insets 2); angular dependence of the low lying levels in the region of anti-crossing (insets 3 ).

$64 \times 64$ Hamiltonian matrix of a Heisenberg ring of $N=6$ spin-1/2 atoms can be reduced to submatrices that are no larger than $3 \times 3[37 \mathrm{~b}, \mathrm{c}]$. A special description of the problem of spin-rings is out of the scope of this article in which we will consider only two examples of POM in order to illustrate the symmetry based computational tools.

In this section, we consider the hexa-nuclear spin rings $\mathrm{Cu}_{6}$ and $\mathrm{Mn}_{6}$ encountered in the two polyoxotungstate salts, $\left(n-\mathrm{BuNH}_{3}\right)_{12}\left[(\mathrm{CuCl})_{6}\left(\mathrm{AsW}_{9} \mathrm{O}_{33}\right)_{2}\right] \cdot 6 \mathrm{H}_{2} \mathrm{O}$ and $\left(n-\mathrm{BuNH}_{3}\right)_{12}$ $\left[(\mathrm{MnCl})_{6}\left(\mathrm{SbW}_{9} \mathrm{O}_{33}\right)_{2}\right] \cdot 6 \mathrm{H}_{2} \mathrm{O}$ [58a] in order to illustrate exploration of spin-symmetry and ITO approach for the analysis of the isotropic and anisotropic exchange interactions in spin rings. Consideration of spin multiplets and related magnetic properties of these chain-like molecular magnets based on $\mathrm{Cu}^{2+}$ and $\mathrm{Mn}^{2+}$ provides a good basis for the illustration of the approach and for identifying common properties of magnetic chains from the 
(a)

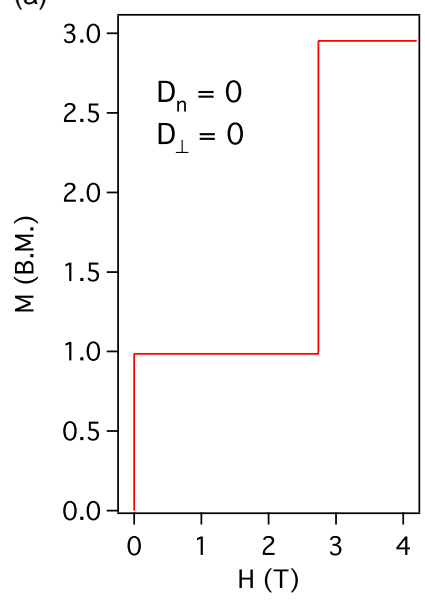

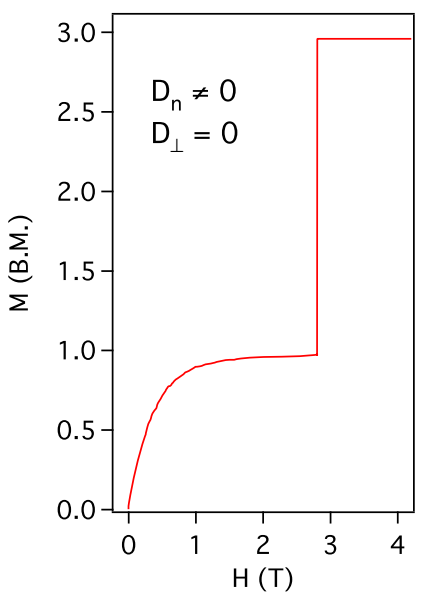

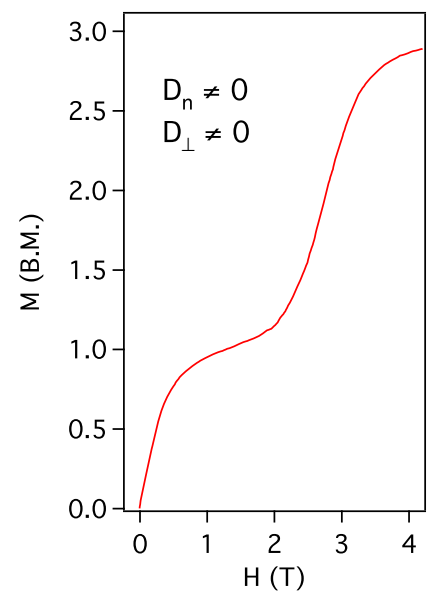

(b)

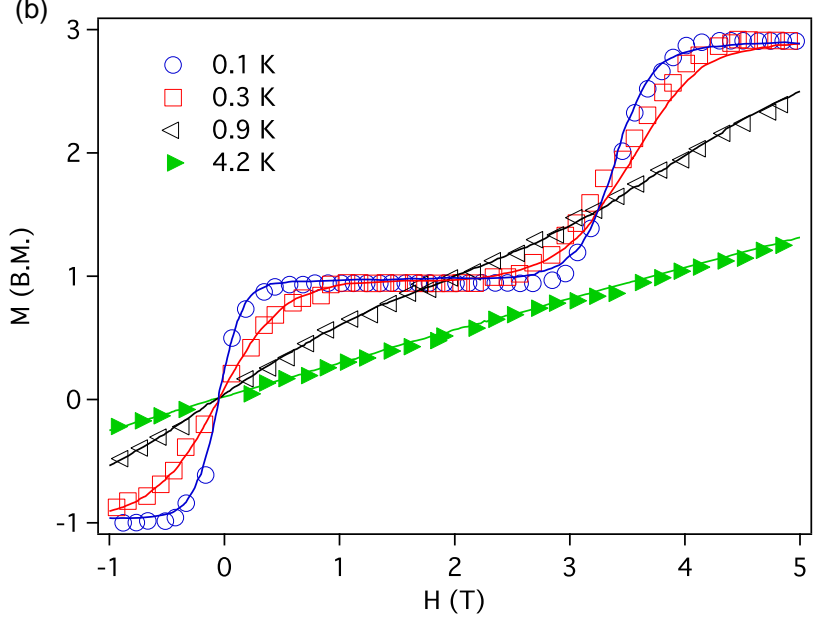

Figure 5. Illustration for the influence of the two components of the AS exchange on the shape of stepwise function of magnetisation vs field in $\mathrm{V}_{15}$ (a) and fit of the theoretical model to the experimental data (b).

symmetry point of view. X-ray single-crystal analysis, magnetic characterisation and discussion of the properties of these compounds are given in Refs. [58b,c] The complexes have $\mathrm{D}_{3 \mathrm{~d}}$-symmetry, and six 5-fold coordinated metal ions which have approximately hexagonal arrangement of the types of on $\mathrm{Cu}_{6}^{2+}$ and $\mathrm{Mn}_{6}^{2+}$ (abbreviated hereafter $\mathrm{Cu}_{6}$ and $\mathrm{Mn}_{6}$ ) as demonstrated in Figure 6. It is important to note, that the metal ion in these compounds are subjected to the action of a low symmetry crystal field which fully removes the orbital degeneracy of the electronic shells.

\subsubsection{Analysis of the exchange interactions in polyoxometalates $\mathrm{Cu}_{6}$ and $\mathrm{Mn}_{6}$, magnetic anisotropy}

The analysis of the isotropic and anisotropic exchange interactions can be performed according to the methodology discussed is the previous Section. First, the isotropic part of the exchange Hamiltonian is assumed to play the leading role. Since the degeneracy 


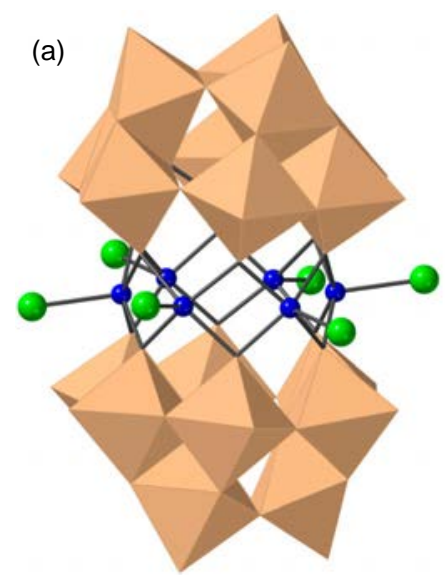

(b)

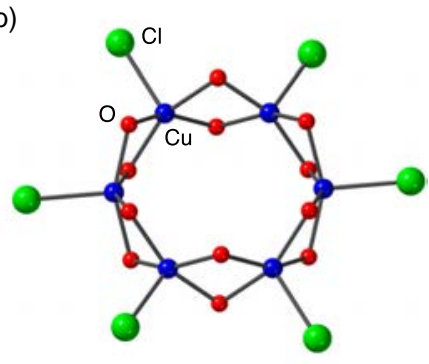

Figure 6. Structure of $\mathrm{Cu}_{6}$ clusters according to [58]: general view (a); $\mathrm{Cu}_{6}$ ring (b). The structure for the $\mathrm{Mn}_{6}$ compound is closely related.

of the electronic terms of the metal ions is lifted by the low symmetry crystal field, the exchange interaction can be described by the isotropic HDVV model. The HDVV Hamiltonian including only the dominating nearest neighbour interactions can be represented as

$$
H=-2 J_{0}\left(S_{1} S_{2}+S_{2} S_{3}+S_{3} S_{4}+S_{4} S_{5}+S_{5} S_{6}+S_{6} S_{1}\right) .
$$

In Equation (13) the parameter $J_{0}$ describes exchange interactions between the spins of the neighbouring metal sites, $S_{i}=1 / 2$ for $\mathrm{Cu}^{2+}$ ions and $S_{i}=5 / 2$ for $\mathrm{Mn}^{2+}$. The magnetic behaviour of these magnetic clusters undoubtedly shows that the exchange coupling is ferromagnetic [58]. Then, following the procedure described in Section 2.1.4 one can find the allowed spin states resulting from spin coupling. For example, for $\mathrm{Cu}_{6}$ the set of spin states is the following:

$$
D^{(1 / 2)} \otimes D^{(1 / 2)} \otimes D^{(1 / 2)} \otimes D^{(1 / 2)} \otimes D^{(1 / 2)} \otimes D^{(1 / 2)}=5 D^{(0)}+9 D^{(1)}+5 D^{(2)}+D^{(3)} .
$$

For this cluster the problem is rather simple due to small dimension of the Hilbert space $\left(2^{6}=64\right)$, but it is illustrative in the sense that the use of spin-symmetry allows to reach the block-structure the HDVV Hamiltonian. In fact, as follows from Equation (14) the maximal size of the block is 9 , which occurs for the total spin $S=1$. The case of $\mathrm{Mn}_{6}$ chain is more complicated due to larger Hilbert space which comprises $6^{6}=46,656$ states for six spins $S_{i}=5 / 2$. A precise solution of the HDVV problem, Equation (13), can be found for both systems with the use of software in Ref. [41]. The evaluated energy patterns for $\mathrm{Cu}_{6}$ and $\mathrm{Mn}_{6}$ are shown in Figures 7 and 8 respectively. In both cases the ground state is ferromagnetic with $S_{\max }=6$ and $S_{\max }=15$, for $\mathrm{Cu}_{6}$ and $\mathrm{Mn}_{6}$, respectively. It is remarkable that the pattern of the spin levels of $\mathrm{Mn}_{6}$ clearly exhibits the so-called rotational structure (marked by the dashed line in Figure 8). The concept of rotational structure of the levels in the spectra of closed spin chains has been intensively discussed and explained in physical terms in [59].

Using the group-theoretical classification, one can establish the correlation between the spin multiplets $n(S) D^{(S)}$ and the terms $S \Gamma$ of $\mathbf{C}_{6 \mathrm{v}}$ point group. Omitting the details 

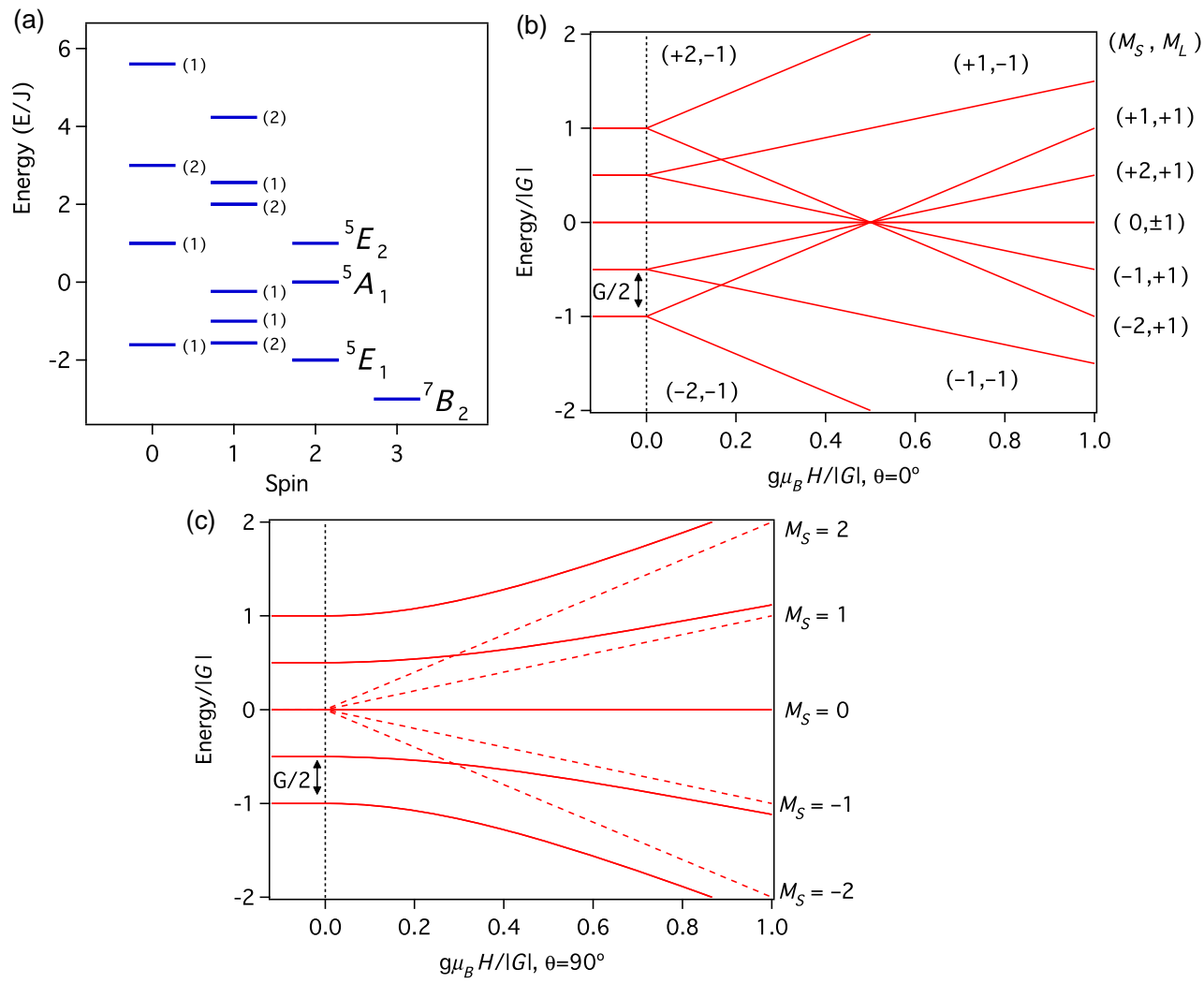

Figure 7. Energy levels of the $\mathrm{Cu}_{6}$ ring ordered in stacks according to total spin values (a). The symmetry labels for $S=2$ and $S=3$ are shown. The remaining multiplicities of the spin states are indicated in parentheses; (b) splitting of ${ }^{5} E_{1}$ level (labelled by the pseudo-angular momentum projections) by AS exchange and Zeeman interaction in the parallel field $\left(\boldsymbol{H} \| \boldsymbol{C}_{6}\right)$; (b) splitting of ${ }^{5} E_{1}$ level in the perpendicular field $\left(H \perp C_{6}\right)$.

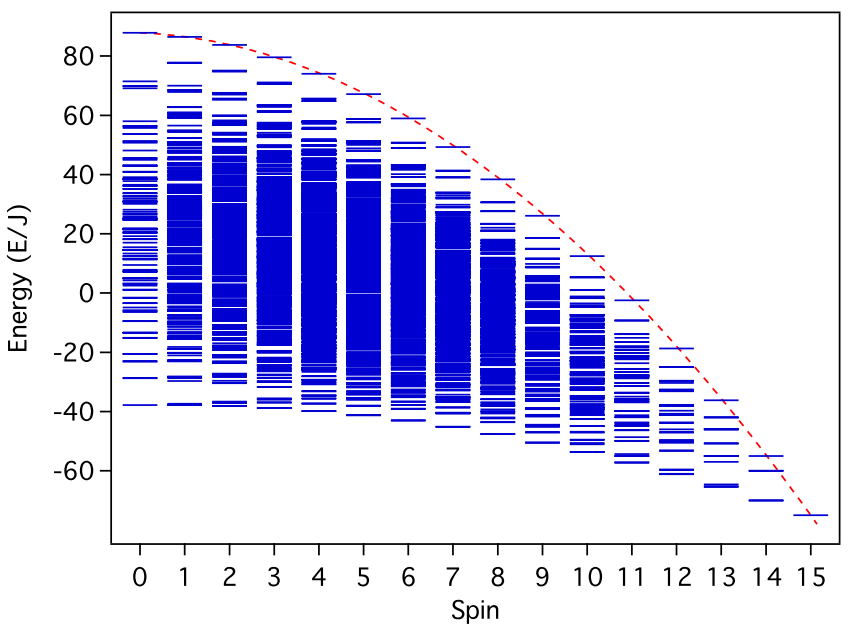

Figure 8. Energy pattern for the ferromagnetic $\mathrm{Mn}_{6}$ ring. The levels are ordered in stacks according to the total spin values. The shape of the rotational band is marked by the dashed line. 
of the procedure so far discussed (see Section 2.1) one can symbolically express the $n(S) D^{(S)} \leftrightarrow S \Gamma$ correspondence for the $\mathrm{Cu}_{6}$ ring as

$$
\begin{aligned}
& D^{(3)} \rightarrow{ }^{7} B_{2}, \\
& 5 D^{(2)} \rightarrow{ }^{5} A_{1}+{ }^{5} E_{1}+{ }^{5} E_{2}, \\
& 9 D^{(1)} \rightarrow{ }^{3} A_{2}+2^{3} B_{2}+{ }^{3} E_{2}+2^{3} E_{1}, \\
& 5 D^{(0)} \rightarrow 2^{1} A_{1}+{ }^{1} B_{1}+{ }^{1} E_{1}
\end{aligned}
$$

Assignation of the spin states to the exact terms (i.e. total spin and irrep) of the system contains full information for the analysis of the energy pattern, magnetic anisotropy and other properties interrelated with symmetry. One can see that the energy pattern (Figure 7a) comprises orbitally degenerate states (irreps $E_{1}$ and $E_{2}$ in $\mathbf{C}_{6 \mathrm{v}}$ ) and orbital singlets $A_{1}, A_{2}, B_{1}, B_{2}$. For example, nine levels with $S=1$ involve three orbital singlets ${ }^{3} A_{2}, 2^{3} B_{2}$ and the orbital doublet ${ }^{3} E_{2}$. The set of the terms involves orbital doublets (irreps $E_{1}$ and $E_{2}$ ), for example, first and second $S=1$ levels for both $\mathrm{Cu}_{6}$ and $\mathrm{Mn}_{6}$. These degeneracies can be referred to as 'exact' degeneracies because they arise from the hexagonal point symmetry of the system and, thus, cannot be removed by the remaining isotropic interactions (such as HDVV interactions between remote spins or biquadratic exchange). The matrix of the HDVV Hamiltonian for $\mathrm{Cu}_{6}$, has block-structure in the symmetry adopted basis. This matrix contains only two $2 \times 2$ sub- matrices corresponding to $2^{3} B_{2}$ and $2^{1} A_{1}$ terms, so that the full eigen-problem for the six-membered s-1/2 ring admits simple analytical solution. Moreover, since in the $S=2$ manifold there no repeating irreps, the HDVV matrix becomes immediately diagonal in $S \Gamma$ representation with the $S \Gamma$-blocks being one-dimensional. One can see that full exploration of symmetry in this case has advantage compared to the approach proposed in Refs. [37b,c].

The dimensions of $S \Gamma$ blocks for the $\mathrm{Mn}_{6}$ ring are much higher so that the analytical expressions for the energy levels are unavailable, while the numerical solution in this case is found through the use of MAGPACK software. For this system the levels are ordered according to the total spin values (Figure 8).

As already mentioned, the group-theoretical assignment allows to elucidate the necessary conditions for the existence and character of the magnetic anisotropy. For the excited states exhibiting orbital degeneracy this can be illustrated by considering the simplest case provided by the $\mathrm{Cu}_{6}$ hexagon for which the $S=2$ manifold involves two orbital doublets, ${ }^{5} E_{1}$ and ${ }^{5} E_{2}$. When the orbital degeneracy is present, the spin-orbital interaction acts as a first order perturbation which splits the ${ }^{5} E_{1}$ and ${ }^{5} E_{2}$ terms. This can be proved using the receipt elaborated in Section 2.1.5 for a triangular system having the orbital doublet ${ }^{2} E$ in the ground state. Note that within these terms the spin-orbital coupling is reduced to the axial form $\lambda L_{Z} S_{Z}$. Alternatively, the only z-component (normal to the plane) of the AS exchange is active and splits the ${ }^{5} E_{1}$ and ${ }^{5} E_{2}$ spin multiplets. As an example, the fine structure of the ${ }^{5} E_{1}$ level ( $G$ is an effective parameter of the AS exchange) is given in Figure $7 \mathrm{~b}$ along with the Zeeman sublevels in parallel field and labels of pseudo-angular momentum quantum numbers. Since the system exhibits linear dependence of the levels in parallel field $\left(\boldsymbol{H} \| C_{6}\right)$ it possesses internal magnetic moment linked to this axis. On the contrary, in perpendicular field $\left(H \perp C_{6}\right)$ the field dependence of the energy levels proves to be quadratic at low field that means that the field induces the magnetic moment. At the strong magnetic field the levels become linear in field with respect to the new (in plane) axis of magnetisation as shown in Figure 7c. More detailed study demonstrates very strong 


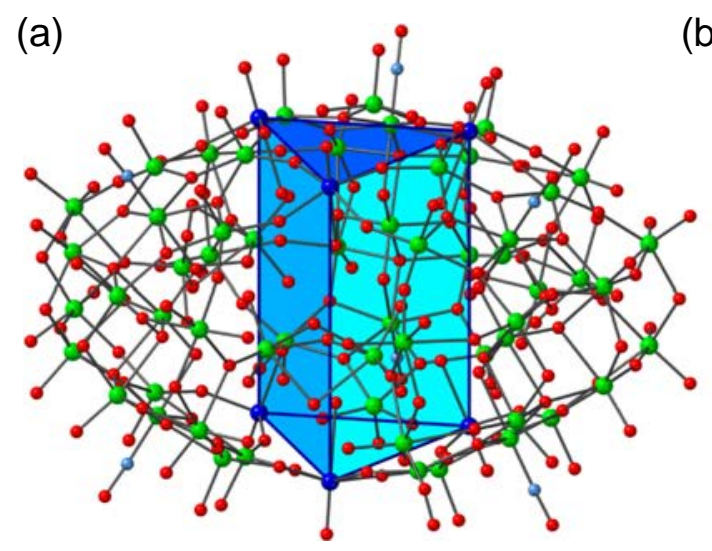

(b)

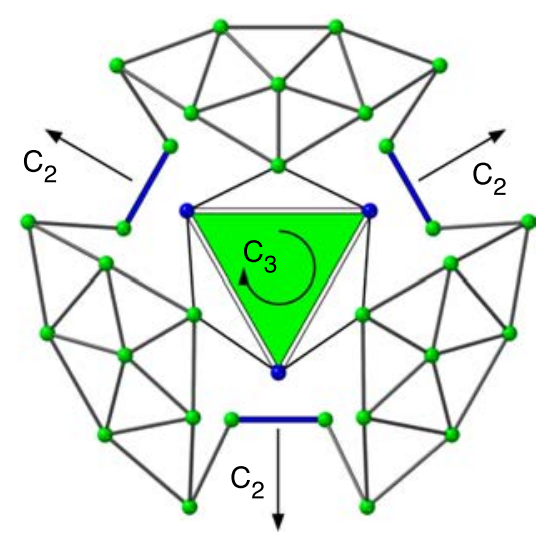

Figure 9. The structure of $\left\{\mathrm{Mo}_{57} \mathrm{M}_{6}\right\}$ cluster in ball-and-stick representation. the triangular $\mathrm{M}_{6}$ spin prism is highlighted (Mo: green, $\mathrm{O}$ : red, $\mathrm{N}$ : light blue, heterometal cations: dark blue). (b): the principal symmetry axes of the metal skeleton (Mo positions of $\left\{\mathrm{Mo}_{17}\right\}$ groups: green, Mo positions of $\left\{\mathrm{Mo}_{2}\right\}$ groups: blue bar, heterometal ions: blue).

magnetic anisotropy interrelated with the AS exchange. It is worth to underline that the conclusion so far made is based solely on the symmetry arguments emanating from the group-theoretical classification of the exchange multiplets. The discussion of the experimental data demonstrates a significant role of the zero-field splitting in the ground orbitally non-degenerate states for both compounds (the details are given in Ref. [58b]).

\subsection{The clusters $\left\{M_{57} M_{6}\right\}\left(M=V^{I V}\right.$ or $\left.M=F e^{I I I}\right)$ : antisymmetric exchange in a biprismatic $V_{6}$ cluster}

\subsubsection{Structure and magnetic sites in $\left\{M_{5} M_{6}\right\}$}

In this section, we use again the group-theoretical approach in order to demonstrate the effects of the AS exchange in a more complex POM such as $\left\{\mathrm{Mo}_{57} \mathrm{M}_{6}\right\}$, whose ball-andstick representation is given in Figure 9. The metal skeleton (highlighted in Figure 9) of $\left\{\mathrm{Mo}_{57} \mathrm{M}_{6}\right\}$ involves six $\mathrm{M}=\mathrm{V}^{\mathrm{IV}}$ or $\mathrm{M}=\mathrm{Fe}^{\mathrm{III}}$ ions as described in Ref. [60]. The prismatic magnetic metal network $\mathrm{M}_{6}$ is formed by the two triangles lying in the parallel planes with a common $C_{3}$ symmetry axis $\left(\mathbf{D}_{3 \mathrm{~h}}\right.$ symmetry) as shown in Figure $9 \mathrm{a}$. Each antiferromagnetic triangle occupies $\mathbf{C}_{3 \mathrm{v}}$ site-symmetry position and possesses ground ${ }^{2} E$ term that is spin frustrated and therefore we are dealing with an interesting and unusual case of two coupled spin frustrated moieties with a common $\mathrm{C}_{3}$ axis.

\subsubsection{Exchange interactions in $\left\{M_{5} \mathrm{M}_{6}\right\}$ and magnetic anisotropy}

The Hamiltonian of the system involving the isotropic HDVV coupling and AS exchange acting within triangular moieties can be represented as

$$
\begin{aligned}
H= & -2 J_{0}\left(S_{1} S_{2}+S_{2} S_{3}+S_{3} S_{1}+S_{4} S_{5}+S_{5} S_{6}+S_{6} S_{4}\right) \\
& -2 J\left(S_{1} S_{4}+S_{2} S_{5}+S_{3} S_{6}\right)-2 J^{\prime}\left(S_{1} S_{6}+S_{3} S_{4}+S_{3} S_{5}+S_{2} S_{6}+S_{1} S_{5}+S_{2} S_{4}\right) \\
& +\sum_{i, k=1,2,3} D_{i k}\left[S_{i} \times S_{k}\right]+\sum_{i, k=4,5,6} D_{i k}\left[S_{i} \times S_{k}\right],
\end{aligned}
$$


(a)

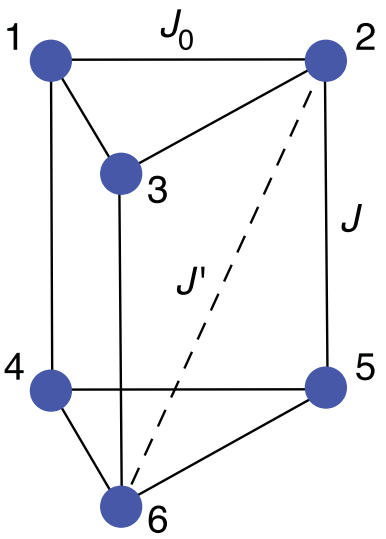

(b)

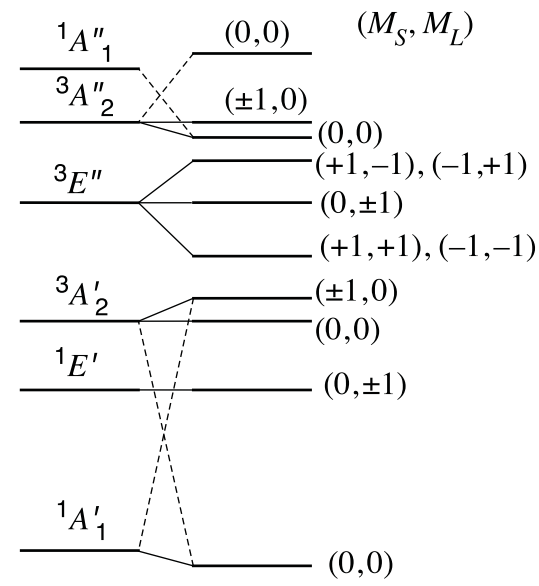

Figure 10. Metal network of biprismatic $\mathrm{V}_{6}$ cluster and scheme of the isotropic exchange parameters (a); energy scheme of $V_{6}$ with the indication of the point symmetry labels and pseudo-angular momentum quantum numbers (b).

where the intra-triangle (with the coupling parameter $J_{0}$ ) and two types of inter-triangle isotropic exchange interactions (with the coupling parameters $J$ and $J^{\prime}$ ) and are taken into account and $\boldsymbol{D}_{i k}$ are the pairwise AS vector parameters defined as in Section 2.1. The scheme of the isotropic exchange interactions is shown in Figure 10a.

A remarkable feature of the exchange network in $V_{6}$ is that the inter-triangle isotropic coupling does not reduce the trigonal symmetry of the system and, therefore, does not directly affect spin-frustration in triangular subunits which retain the $C_{3}$ axes. We thus arrive at the problem of AS exchange in the two coupled triangular unit [60]. Since the coupling does not reduce the trigonal symmetry, one can expect that the AS exchange is not suppressed neither in each triangular unit neither in the entire molecule.

To model this $\mathrm{V}_{6}$ cluster we first derive the spin coupling scheme for six $S_{i}=1 / 2$ spins, which determines the allowed spin state of the system, Equation (14). Then, we apply the procedure of the group-theoretical assignation of spin states as described in Section 2.1.4 (here we omit the detail of calculations). As a result, one can obtain the following correlation between the HDVV spin multiplets and the irreps (and exact terms of the system) of the $\mathbf{D}_{3 \mathrm{~h}}$ point group:

$$
\begin{aligned}
& D^{(3)} \rightarrow{ }^{7} A^{\prime \prime}{ }_{2}, \\
& 5 D^{(2)} \rightarrow{ }^{5} A^{\prime}{ }_{1}+{ }^{5} E^{\prime}+{ }^{5} E^{\prime \prime}, \\
& 9 D^{(1)} \rightarrow{ }^{3} A^{\prime}{ }_{2}+2^{3} A^{\prime \prime}{ }_{2}+{ }^{3} E^{\prime}+2^{3} E^{\prime \prime}, \\
& 5 D^{(0)} \rightarrow 2^{1} A^{\prime}{ }_{1}+{ }^{1} A^{\prime \prime}{ }_{1}+{ }^{1} E^{\prime}
\end{aligned}
$$

As an immediate consequence, one can see that the HDVV Hamiltonian has blockstructure in the symmetry adapted basis, with the maximal size of the submatrices being equal to 2 (two pairs $2^{3} A_{2}^{\prime \prime}$ and $2^{1} A_{1}^{\prime \prime}$ ). Notice that the simplification can be achieved even if along the isotropic coupling, the AS exchange is included in the model. Figure 10b shows the low-lying part of the energy pattern related to the coupled triangles in their ground states ${ }^{2} E$. The intra- and inter-triangle isotropic interactions are antiferromagnetic and 
the AS exchange acts within each triangle. One can see that the energy pattern exhibits orbital doublets which are split (providing $S \neq 0$ ) by AS exchange acting as a first order perturbation, while in the orbital singlets with $S \neq 0$ the AS exchange appears as a second order perturbation giving rise to a conventional zero-field splitting. According to the general statements (Section 2.1) each orbital doublet in a trigonal system is labelled by the projections $M_{L}= \pm 1$ of the pseudo-angular momentum, while for the orbital singlets $M_{L}=0$. AS exchange links the states of the system accordingly to the conventional rule of the addition of the angular momenta projections $M_{J}=M_{L}+M_{S}$.

It is advantageous that the pseudo-angular momentum labels (Figure 10b) indicate also the selection rules for the EPR transitions and give a qualitative insight on the magnetic anisotropy. In fact, the ground state proves to be an orbital singlet ${ }^{1} A_{1}^{\prime}$ and so at low temperatures the system is expected to exhibit temperature independent Van Vleck paramagnetism. The orbital doublet ${ }^{1} E^{\prime}$ that is diamagnetic state from the point of view of the HDVV model is expected to exhibit a Zeeman splitting of pure orbital nature. This kind of orbital paramagnetism is fully anisotropic in the sense that the magnetisation is maximal for the field directed along the $\mathrm{C}_{3}$ axis and vanishes when the field is parallel to the planes of triangles. The zero-field splitting for orbital doublet ${ }^{3} E^{\prime \prime}$ gives rise to three doublets labelled by the quantum numbers $M_{J}=0\left(M_{S}= \pm 1, M_{L}=\mp 1\right), M_{J}= \pm 1\left(M_{S}=0, M_{L}= \pm 1\right)$ and $M_{J}= \pm 2\left(M_{S}= \pm 1, M_{L}= \pm 1\right)$, which suggests a strong magnetic anisotropy. Finally, the selection rules for the EPR transitions (which cannot in principle be adequately derived from the HDVV model) are $M_{J}-M_{J}^{\prime}=0, \pm 1$. One can thus conclude that by using only the symmetry arguments (group-theoretical classification in conjunction with the pseudoangular momentum concept), it is possible to explain qualitatively and, in part, quantitatively the observed magnetic anisotropy and EPR spectra of complex systems exhibiting isotropic and anisotropic exchange interactions.

\section{Mixed-valence magnetic polyoxometalates}

\subsection{Reduced mixed valence polyoxovanadates $\left[V_{18} O_{48}\right]^{n-}(n=4 \div 18)$}

\subsubsection{General approach to the electronic problem of mixed valency and double exchange: exploration of spin symmetry}

The consideration in previous Chapters has been essentially based on the assumption (which has not been explicitly mentioned ) that spins are linked to the definite sites in the molecule. Here we proceed to the consideration of the so-called MV compounds which contain one or several electrons that can be delocalised over the network of paramagnetic or diamagnetic metal ions (spin cores or spinless cores) [61]. In this kind of compounds a specific magnetic coupling (referred to as double exchange) between the magnetic ions appears giving rise to a rich variety of physical properties. Double exchange interaction involves the coupling of two localised magnetic moments, having spin cores So, through an itinerant extra electron that can travel forth and back between the two magnetic centres. The ferromagnetic spin alignment is achieved due to the energy gain through the polarisation mechanism as illustrated by the classical spin picture (Figure 11a). In fact, an electron initially coupled to the ion A by the ferromagnetic Hund type exchange, tends to align the spin So of the site B in the same direction. The ground state of a dimer is reached 


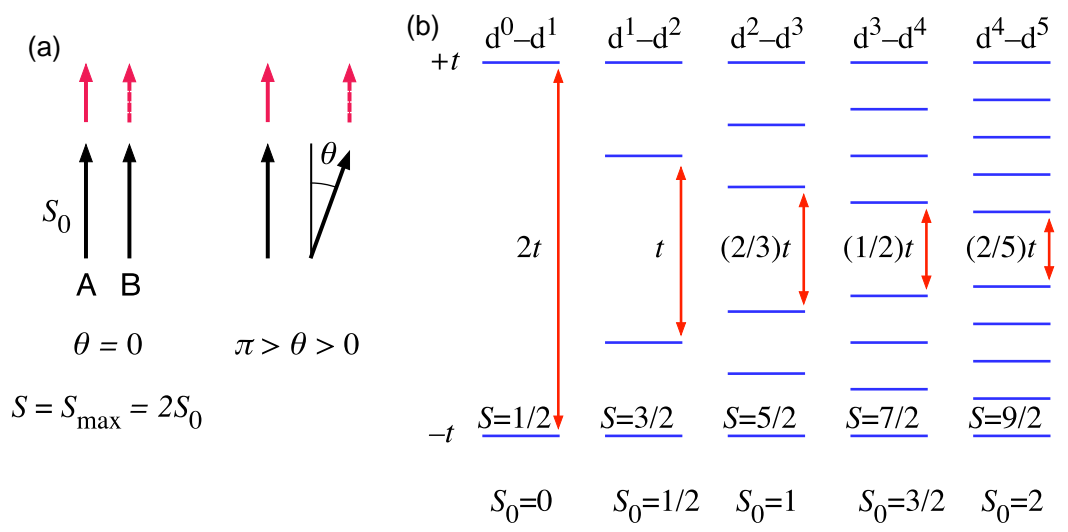

Figure 11. Double exchange, spin polarisation effect, red arrows denote the itinerant spin (a); effect of the double exchange in magnetic dimers: schemes of the energy levels for the series of MV dimers with different spin cores $S_{0}(b)$.

when the three spins (two spins So and the spin of the extra electron) are parallel gaining exchange energy of the system (Figure 11a). The mechanism of the double exchange was suggested to explain the ferromagnetism observed in MV manganites with perovskite structure $\left(\mathrm{La}_{x} \mathrm{Ca}_{1-x}\right)\left(\mathrm{Mn}^{\mathrm{III}} \mathrm{Mn}^{\mathrm{IV}}{ }_{1-x}\right) \mathrm{O}_{3}$. In Ref. [61] it was suggested a solution of the double exchange for a MV dimer for which the following spin dependence of the energy levels was deduced (Figure 11):

$$
E_{ \pm}(S)= \pm \frac{t(S+1 / 2)}{2 S_{0}+1}
$$

where $t$ is the electron transfer integral, and $S$ is the total spin of the system. One can see that the energy levels linearly depend upon the total spin of the dimer and the ground state is ferromagnetic irrespectively of the sign of the transfer parameter. This dependence is drastically different from the quadratic spin dependence (of the type of $S(S+1)$ in simple systems) arising from the HDVV exchange in spin-localised systems. Spin-delocalised MV dimers can be exemplified by the complex $\left[\mathrm{L}_{2} \mathrm{Fe}_{2}(\mu-\mathrm{OH})_{3}\right]\left(\mathrm{ClO}_{4}\right) \cdot 2 \mathrm{CH}_{3} \mathrm{OH}$. $2 \mathrm{H}_{2} \mathrm{O}$ (Refs. $\left.[62,63]\right)$ based on $\mathrm{Fe}^{\mathrm{II}}\left(S_{\mathrm{Fe}(\mathrm{II})}=2\right)$ and $\mathrm{Fe}{ }^{\mathrm{III}}\left(S_{\mathrm{Fe}(\mathrm{III})}=5 / 2\right)$, which exhibit a ferromagnetic ground state and a strong intervalence absorption band. It is important to underline that the basic expression deduced for a MV dimer, Equation (18), is not applicable to more complex systems. Generalisation of the theory demonstrated much more complex and qualitatively different, which is especially important, energy pattern in trimers and high nuclearity MV systems [64,65]. In particular, the basic statement about the ferromagnetic spin alignment in the ground state can be invalid. In this respect, it is to be mentioned that the peculiarities of the so-called frustrated MV systems with triangular electronic topologies, like symmetric trimeric clusters and cubane-type complexes (see review articles [40]) are especially interesting. It is to be noted that the universal analytical expressions for the energy levels for complex MV systems containing more than two magnetic centres and several mobile electrons are unavailable, while evaluation these levels even in particular cases represents rather complicated task. 
A general approach to the problem of double exchange in high nuclearity clusters containing an arbitrary number of localised spins $(N)$ and arbitrary number of itinerant electrons $(P)$ moving among spin sites has been worked out in Ref. [42]. The Hamiltonian of the system within the Hubbard-like model can be written as follows:

$$
H=\sum_{i, k} t_{i k}\left(c_{k \sigma}^{+} c_{i \sigma}+\text { h.c. }\right)+\sum_{i, k} J_{i k} \boldsymbol{s}_{i} \boldsymbol{s}_{k}+\sum_{i, k} U_{i k} n_{i} n_{k}
$$

In Equation (19) the following notations for the parameters of the key interactions are used: $t_{i k}$ are the transfer parameters corresponding to the electronic jumps between sites $i$ and $j, c_{k \sigma}^{+}\left(c_{k \sigma}\right)$ are the creation (annihilation operators) of an electron with the spin projection $\sigma$ at the site $k, U_{i k}$ is the Coulomb repulsion of the electrons instantly localised at the sites $i$ and $k$, the populations $n_{i}$ and $n_{k}$ ( 0 or 1$)$ indicate that only populated sites are involved in the Coulomb repulsion of the electrons. The isotropic exchange interaction between the localised and delocalised spins is also included in the model (second term of the Hamiltonian).

In principle the Hamiltonian, Equation (19) can be treated numerically, but the dimensions of the matrices to be diagonalised increase dramatically with the increase of the number of the ions, spins of sites and the number of the mobile electrons, which restricts the size of the system that can be really treated. Application of spin symmetry can essentially enlarge the ability of the approach to treat the high nuclearity systems encountered in POM chemistry.

In order to apply spin symmetry arguments to a MV system let us imagine that the itinerant electrons are instantly localised at a set of definite sites. Let us further suppose that the spins of the instantly localised system are coupled in a successive way to give the total spin $S$ of the cluster. Let us use the chain-like coupling scheme in which the spins are successively coupled (such as in Equation (2)). This coupling scheme can be expressed as

$$
(\tilde{S}) \equiv\left\{\tilde{S}_{2}, \tilde{S}_{3}, \ldots \tilde{S}_{N-1}\right\}
$$

with the possible sets of the intermediate spins $\left(\tilde{S}_{2} \equiv S_{12}, \tilde{S}_{3} \equiv S_{123}\right.$, etc. $)$. Note that the intermediate spins depend on the instant distribution of the itinerant electrons as shown in Figure 12. The itinerant spins can be distributed over the magnetic sites of the system, so that the total number of electronic distributions is equal to $N ! / P !(N-P)$ !. The mathematical procedure developed in Refs. [42a,b] (we omit here the mathematical details) allows to evaluate the matrix element of the Hubbard type Hamiltonian corresponding to the electronic jumps $i \rightarrow k$ :

$$
\begin{aligned}
& \mid\left(s_{\beta}, \beta \neq i, k\right)\left(s_{i}=s_{0}+\frac{1}{2}, s_{k}=s_{0}\right)(\tilde{S}) S M_{S} \\
& \quad \rightarrow \mid\left(s_{\beta}, \beta \neq i, k\right)\left(s^{\prime}{ }_{i}=s_{0}, s^{\prime}{ }_{k}=s_{0}+\frac{1}{2}\right)\left(\tilde{S}^{\prime}\right) S M_{S}
\end{aligned}
$$

The jumps between the sites $i$ and $k$ occur with the constant populations of the remaining sites as pictorially shown in Figure 12 illustrating two involved distributions linked via one-electron jump. The non-vanishing matrix elements are expressed in terms of the well-known and tabulated (or, alternatively, programmable) 6j-symbols of the spherical symmetry group; parametrically, the matrix elements depend on the transfer integrals $t_{i k}$. For the sake of simplicity we leave outside of this presentation the explicit expressions (which are rather complicated) for the matrix elements (they are given in Refs. [42a,b]). 


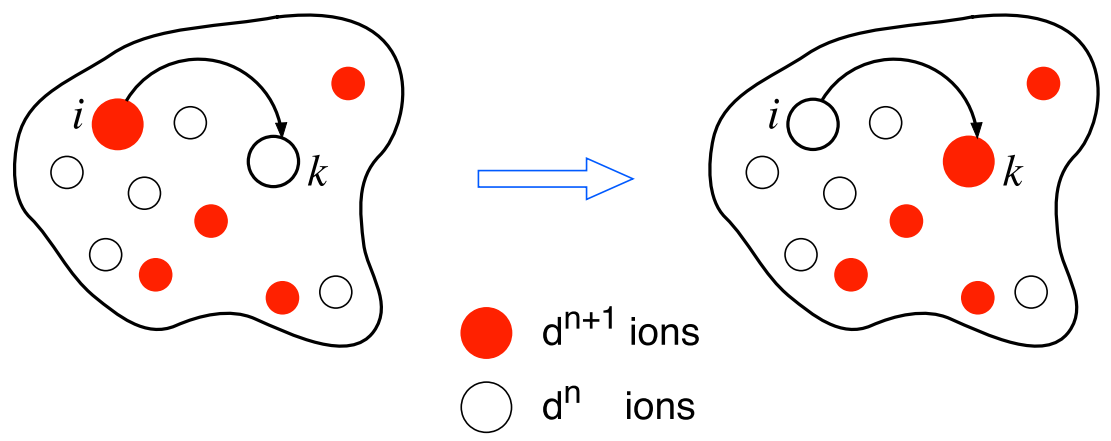

Figure 12. Illustration for a transfer process between sites $i$ and $k$ in a complex MV system containing localised and delocalised spins.

This theoretical approach constitutes the background of the computing package MVPACK [42c] aimed at the calculation of the energy levels and thermodynamic properties of the high nuclearity MV systems combining in their structures localised and delocalised subunits and arbitrary numbers of metal sites and itinerant electrons. MVPACK permits to treat either double exchange systems (i.e. MV systems in which extra electrons travel over the network of spin cores), as well as MV systems comprising spinless cores. In this respect it is worth noting that the majority of reduced POMs just belong to the class of MV systems in which the itinerant electrons are delocalised over spinless cores. The specific spin-dependent mechanism of the electron transfer in these systems arises from the correlated motion of the electrons over spinless ions which is different from double exchange (see discussion in Section 3.2). This program is a further development and generalisation of the package MAGPACK, which is applicable to large exchange coupled clusters comprising only localised spins.

\subsubsection{Magnetic properties of $\left[\mathbf{V}_{\mathbf{1 8}} \mathbf{O}_{\mathbf{4 8}}\right]^{\text {n- }}$ cluster}

As an extremely complex example of a high nuclearity MV POMs we briefly consider the $\left[\mathrm{V}_{18} \mathrm{O}_{48}\right]^{n-}$ family. A detailed description of its structure, together with the exchange network, is given in Figure 13. These POM compounds can serve as redox sponges, in the sense that the electron population inside the cluster can be chemically controlled. Depending on the number of electrons, the family of compounds includes the spin-localised cluster $\left[\mathrm{V}_{18} \mathrm{O}_{42}\right]^{12-}$ formed by 18 oxovanadium (IV) sites (i.e. 18 unpaired electrons localised over the 18 metal sites), $\mathrm{MV}$ compounds oxidised by two electrons, $\left[\mathrm{V}_{18} \mathrm{O}_{42}\right]^{10-}$ (i.e. 16 unpaired electrons delocalised over the 18 metal sites) and MV compounds oxidised by eight electrons, $\left[\mathrm{V}_{18} \mathrm{O}_{42}\right]^{4-}$ (i.e. 10 unpaired electrons delocalised over 18 metal sites, see full description in Ref. [66]).

This family of polyoxovanadates exhibit an unexpected magnetic behaviour illustrated by Figure 14 [67]. Thus, while the effective distances between unpaired electrons are longer in compounds with smaller number of unpaired electrons, thus, favouring weaker antiferromagnetic exchange interactions, one observes that it is precisely the cluster $\left[\mathrm{V}_{18} \mathrm{O}_{42}\right]^{4-}$ the one that exhibits the strongest antiferromagnetic exchange interactions (Figure 14). This shows that the magnetic properties of this series of POMs cannot be properly interpreted in the framework of model dealing with the magnetic exchange between localised 

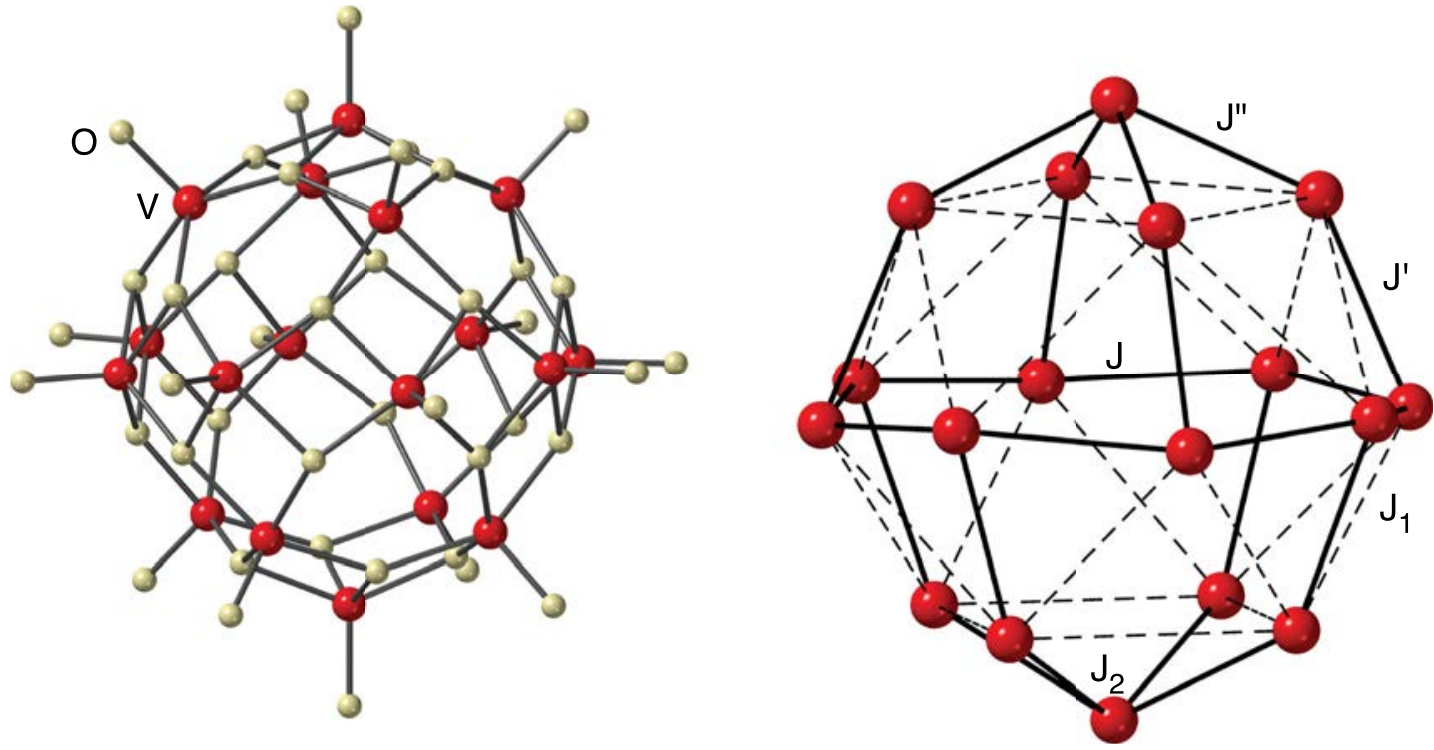

Figure 13. Structure of $\left[\mathrm{V}_{18} \mathrm{O}_{48}\right]^{n-}$ : general view (a) and skeleton of the vanadium sites with the indication of the exchange network (b).
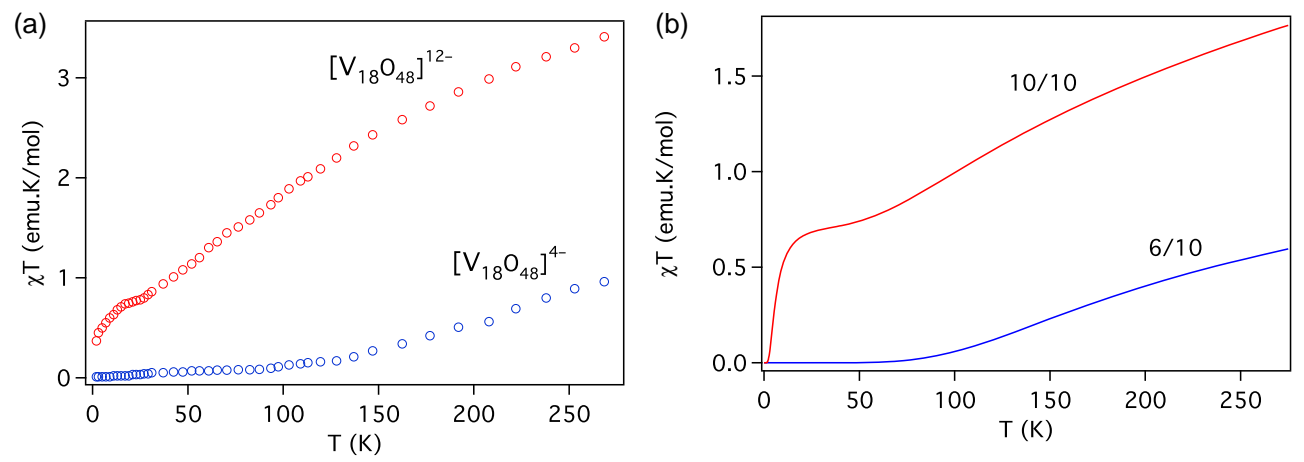

Figure 14. (a) Temperature dependence of $\chi T$ for the spin-localised $\mathrm{V}_{18} \mathrm{IV}$ and $M V \mathrm{~V}_{10}{ }^{\mathrm{IV}} \mathrm{V}_{8} \mathrm{~V}$ clusters (from Ref. [67]); (b) Theoretical magnetic behaviour of a MV spin-delocalised antiferromagnetic cluster in which the electron population has been reduced (from Ref. [66]).

spins. The general approach described in Section 3.1.1 (realised as MVPACK software) was employed to explain such unexpected result.

Thus, as can be seen in Figure $14 \mathrm{~b}$ the magnetic behaviour exhibited by the MV $\left[\mathrm{V}_{18} \mathrm{O}_{42}\right]^{4-}$ cluster can be modelled taking into account the spin delocalisation over the cluster structure, as this leads to a significant stabilisation of the antiferromagnetic ground spin state with respect to the other excited spin states (for the modelling see details in Ref. [66]). It is worth to notice that this is the most complex problem addressed so far in MV magnetic POMs and moreover in the entire area of MV molecular clusters. 

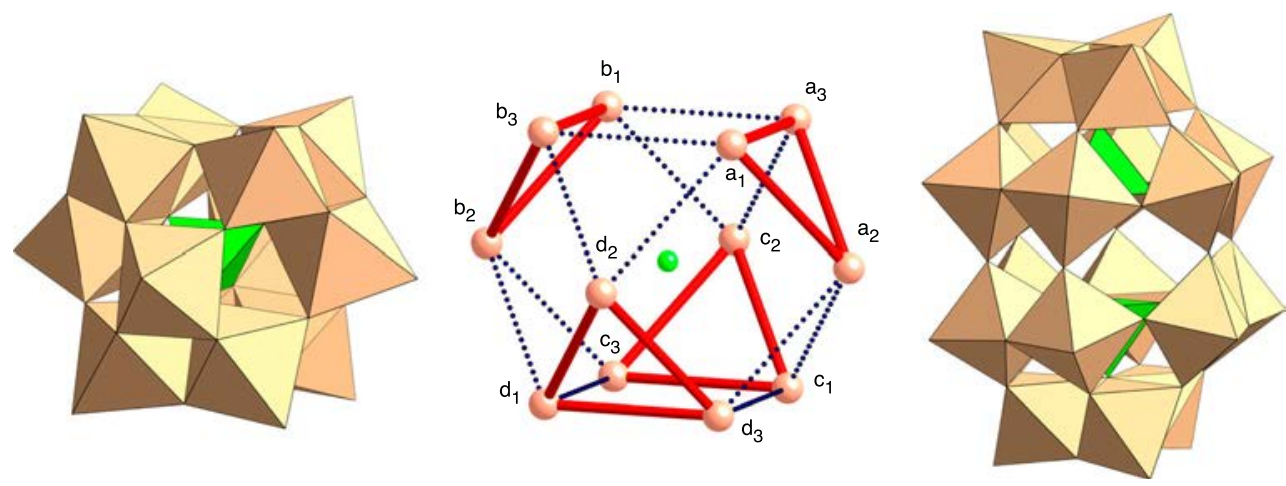

Figure 15. Two structures of the two-electron reduced polyoxometalates with delocalised electronic pairs: Keggin structure of a $\left[\mathrm{XM}_{12} \mathrm{O}_{40}\right]^{n-}$ anion (a); metal network of the Keggin anion (b); Wells-Dawson structure $\left[\mathrm{X}_{2} \mathrm{M}_{18} \mathrm{O}_{62}\right]^{n-}$ (c), where $\mathrm{M}=\mathrm{Mo}, \mathrm{W}$. In the Keggin anion the two types of electron transfer processes between neighbouring $\mathrm{WO}_{6}$ octahedra (through edges, $\mathrm{P}$, and corners, $\mathrm{t}$ ) are shown.

\subsection{Mixed-valence polyoxometalates featuring delocalisation of an electronic pair: Keggin and Wells-Dawson anions reduced by two electrons}

\subsubsection{Structures of the Keggin and Wells-Dawson anions}

Keggin and Well-Dawson anions are archetypical structures in POM chemistry (Figure $15 \mathrm{a}, \mathrm{b}, \mathrm{c})$. The Keggin structure consists of four edge-sharing triads of $\mathrm{MO}_{6}$ units $(\mathrm{M}=\mathrm{W}$, $\mathrm{Mo})$ arranged around the heteroatom $\mathrm{X}(\mathrm{X}=\mathrm{B}, \mathrm{Si}, \mathrm{P}, \mathrm{Co}, \mathrm{Cu}$, etc.) by sharing corners. As one can see from Figure 15a the twelve $\mathrm{MO}_{6}$ units are arranged in such a way that the system possesses an overall tetrahedral symmetry $\mathbf{T}_{\mathrm{d}}$. Figure $15 \mathrm{~b}$ shows the idealised scheme of the metal sites in the Keggin system that consists of the four metal triangles $\left(a_{1} a_{2} a_{3}, b_{1} b_{2} b_{3}\right.$, etc) perpendicular to the $C_{3}$ axes of the tetrahedron. The Wells-Dawson structure consists of $18 \mathrm{MO} 6$ units arranged in two central hexagonal belts with alternating corner and edge connectivity and two triangular caps of MO6 units shearing edges. The four rings (belts and caps) are linked by corners and they give rise to a structure with $\mathrm{D}_{3 \mathrm{~h}}$ symmetry.

Heteropolyanions with these two structures can be reversibly reduced by adding one or more electrons, which are delocalised over the metal network. In this respect, the doublyreduced systems are of special interest due to its unusual magnetic properties, which show a total spin pairing of the 2 electrons even at room temperatures. Actually, the two electrons travelling over the metal sites in Keggin (and Wells-Dawson) anion are far enough from each other due to Coulomb repulsion. Under this condition the exchange interaction is expected to be negligibly small and therefore cannot give rise to the observed strong antiferromagnetic coupling. As a matter of fact, in Refs. [68a,c] it was suggested that the electron delocalisation results in a very efficient stabilisation of the antiferromagnetic spin state of the electronic pair in these anions. The difference between these two types of anions is that in the Keggin anions the two extra electrons are delocalised over all metal sites, while for the Wells-Dawson anion such electron delocalisation is restricted to the two internal hexagonal belts [69]. This physical conclusion is rather general and valid also for other 2e-reduced POMs. Here we will focus on the 2e-reduced Keggin anion to show how specific analytical symmetry-based methods can be useful to account for these unexpected properties. 


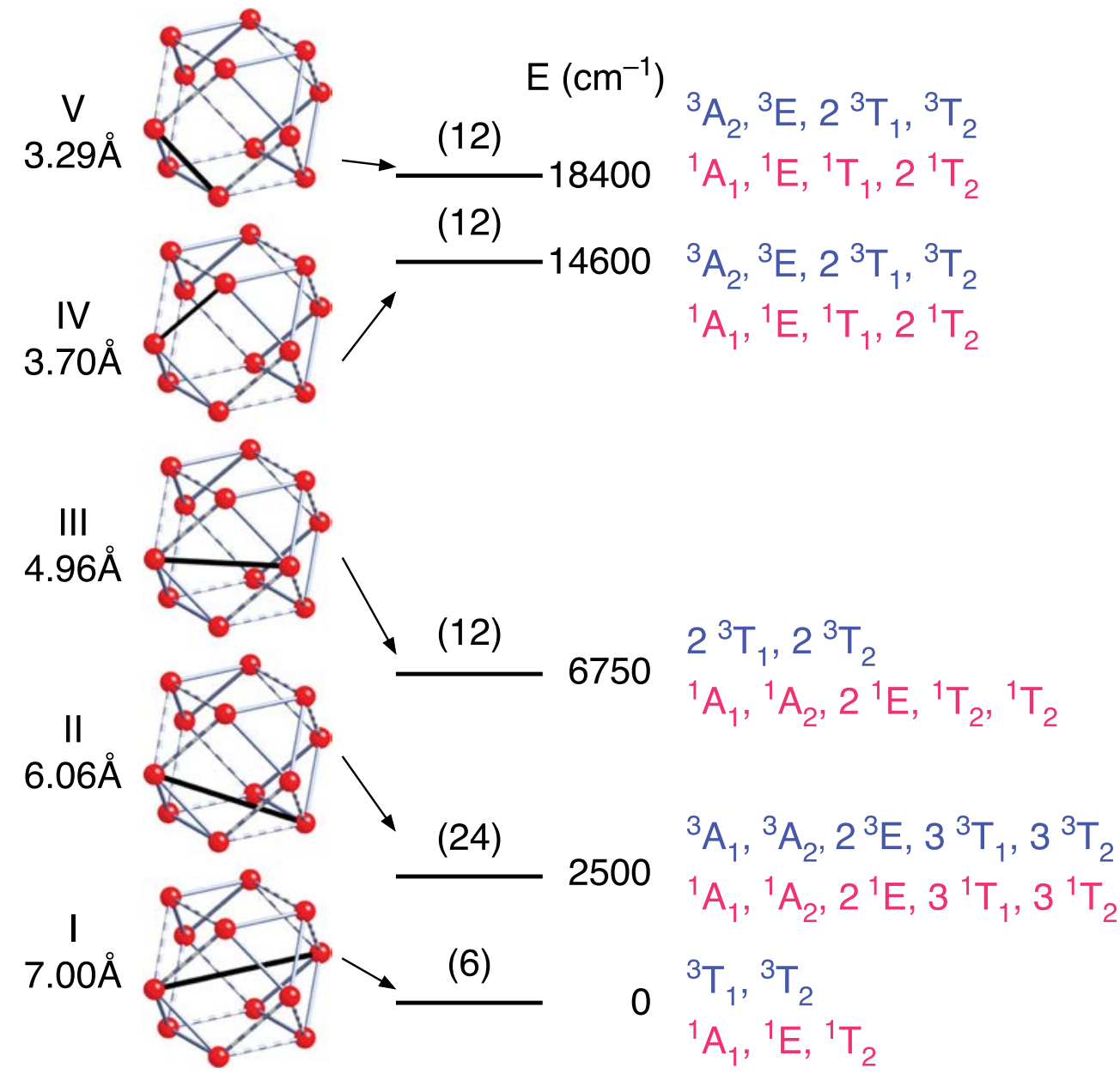

(a)

(b)

(c)

Figure 16. Electronic pairs in Keggin structure: network of the octahedral sites (red balls) with indication of the localizations of the five types of electronic pairs and distances between occupied sites connected by black lines (a); Coulomb energies of the five types of electronic pairs in Keggin structure. The numbers of the equivalent positions for each type of the pair is given in parentheses (b); symmetry labels, spinsinglets-red, spin-triplets-blue (c). Colour online.

\subsubsection{Site symmetry approach: delocalised electronic pair in the 2e-reduced Keggin anion}

Delocalisation of the electronic pair in MV POMs represents a concerted motion of the two electrons over the network of the spinless cores affected by Coulomb repulsion of the electrons. The central part of the adequate consideration of this motion is the site-symmetry approach specially developed as applied to the present problem. Usually the Coulomb repulsion is the leading interaction which can be approximately taken into account within the point charge model, neglecting covalent effects. Although this model is rather simplified and overestimates the Coulomb repulsion it gives a good qualitative (and to some 
extent quantitative) picture of the energy levels interrelated with the structure of the metal network [68]. In the Keggin structure the states of the electronic pair can be classified from I to $\mathbf{V}$ according to the five independent distances between the occupied sites as shown in Figure 16a. The distances in these five types of the electronic pairs vary from $7.00 \AA$ for the pair I to $3.29 \AA$ for the pair V (Figure 16a,b). Each type of the pair has a definite Coulomb repulsion (evaluated within the point charge model) indicated in Figure 16b. Due to overall tetrahedral symmetry, $\mathbf{T}_{\mathrm{d}}$, of the molecule each type of the pair has several positions in the metal network as shown in Figure 16. Thus, the pair I (lowest in energy) has 6 positions (Figure 16b) in which the electronic pair can occur in spin-singlet and spin-triplet states. This picture of localised states of the Keggin anion should now be complemented by the inclusion of the M-M electron transfer processes occurring through the oxo groups. This result in the separation of spin-singlet and spin-triplet terms and mixes states belonging to different pairs with the same symmetry labels. The symmetry labels for these states can be found through the application of the site-symmetry approach adapted to the electronic pair developed in Ref. [68a]. Usually the site-symmetry approach was used for the classification and construction of hybrid orbitals in molecules. A relevant definition of the site-symmetry of an electronic pair allows to perform the group-theoretical classification of the delocalised states of the electronic pair in a complex molecular network.

We define the site-symmetry of a pair in $\boldsymbol{T}_{\mathrm{d}}$ molecule as a site-symmetry of the side in the tetrahedron in the Keggin unit. Site-symmetry of each site is thus $\boldsymbol{C}_{\mathbf{2}}$ and one can assign six local coordinates to six faces of the cube defining thus the instant positions of the pair that can be specified by the number of the face. The wave-functions of the pair I belong to the irreps of $C_{2 \mathrm{v}}$ and can be expressed in terms of Slater determinants (for $a_{2} b_{2}$ position) and to the total spins $S_{p}=1$ and $S_{p}=0$ as

$$
\varphi_{I}\left(\left[a_{2} b_{2}\right] ; S_{p}=1, M=1\right)=\left|a_{2} b_{2}\right|, \varphi_{I}\left(\left[a_{2} b_{2}\right] ; S_{p}=0\right)=\frac{1}{\sqrt{2}}\left(\left|a_{2} \bar{b}_{2}\right|+\left|\bar{a}_{2} b_{2}\right|\right) .
$$

It can be easily seen that these functions belong to the irreps $B_{1}$ and $A_{1}$ of $C_{2 \mathrm{v}}$ and consequently give terms ${ }^{3} B_{1}$ and ${ }^{1} A_{1}$. Each of four pairs II does not belong to the $C_{2 \mathrm{v}}$ site group, but the part of the full Hamiltonian involving two triangles that are associated with the $a b$ side is invariant under the transformation of $C_{2 \mathrm{v}}$. This allows to construct the basis functions of $\boldsymbol{C}_{2 \mathrm{v}}$ from four localised functions of the pair II. This procedure can be extended (see details in Ref. [68]) and all irreps $\Gamma_{0}$ for the localised electronic pairs can be obtained. For example, for the pairs I, II associated with the $a b$ side, one finds: ${ }^{3} B_{1},{ }^{1} A_{1}$ for pair I and ${ }^{1} A_{1},{ }^{3} A_{2},{ }^{3} B_{1},{ }^{3} B_{2},{ }^{1} A_{1},{ }^{1} A_{2},{ }^{1} B_{1}$ for pair II. Applying symmetry operations of the full symmetry $T_{\mathrm{d}}$ group to each basis function belonging to a certain term $S \Gamma_{0}$ of the site group $C_{2 \mathrm{v}}$, one can generate the reducible six-dimensional reducible representations $\Gamma_{f}$ of $\boldsymbol{T}_{\mathrm{d}}$. By decomposing $\Gamma_{f}$ into irreps, one can classify the delocalised states of the pairs according to the irreps $\Gamma$ of the symmetry point group of the system. The results for the pairs I-V are shown in Figure 16c. For example, one can see that the six-dimensional orbital space for the pair I (six positions) gives rise to three spin-singlets ${ }^{1} A_{1}$ (orbital singlet), ${ }^{1} E$ (orbital doublet), ${ }^{1} T_{2}$ (orbital triplet) and two spin-triplets ${ }^{3} T_{1},{ }^{3} T_{2}$.

Then, the delocalisation of the electronic pair splits the Coulomb multiplets according to the irreps of the full symmetry group and total spin of the systems. One can see that the full $66 \times 66$-matrix can be reduced into submatrices (blocks) of smaller sizes accordingly to the numbers of repeated irreps. For the spin triplet and spin-singlet terms we get the 


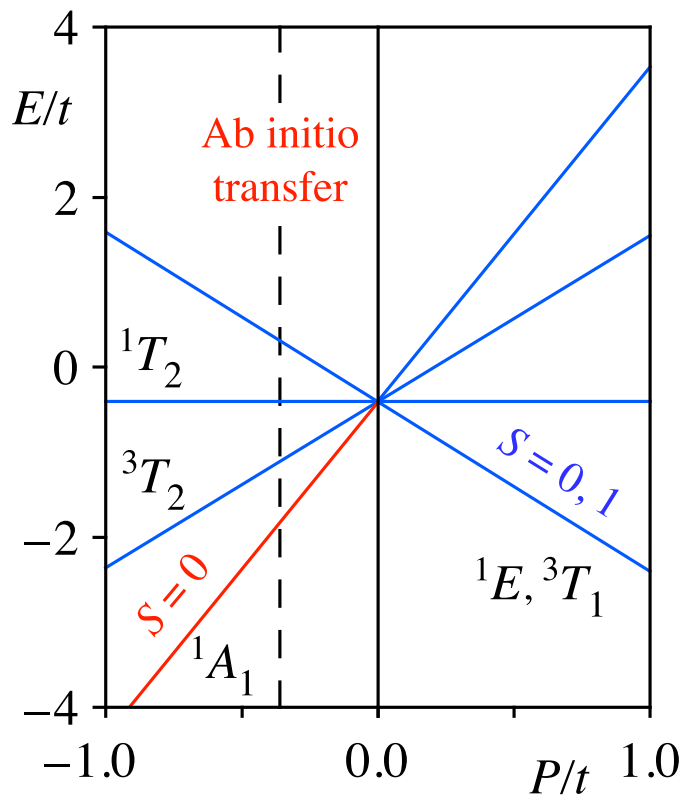

Spin-pairing Spin-mixture

Figure 17. Energy pattern of the pair I as function of transfer parameters $t$ and $\mathrm{P} . \mathrm{t}$ is electron transfer (hopping) parameter between corner-sharing MO6 octahedra, $t^{\prime}$ is electron transfer parameter between edge-sharing octahedra and $P$ is a two-electron transfer parameter one between corner-sharing and other between edge-sharing. In this figure the ratio $t^{\prime} / t$ is fix to 1 .

following sets of irreps:

$$
\begin{aligned}
& D^{\left(S_{p}=1\right)}(66)={ }^{3} A_{1}+3^{3} A_{2}+4^{3} E+10^{3} T_{1}+8^{3} T_{2}, \\
& D^{\left(S_{p}=0\right)}(66)=5^{1} A_{1}+2^{1} A_{2}+7^{1} E+7^{1} T_{1}+8^{1} T_{2} .
\end{aligned}
$$

Therefore, the maximal dimension of the energy matrix for spin-triplet terms is $10 \times 10$ while for spin-singlet this dimension is $8 \times 8$. The computational problem can be further simplified if one assumes that the Coulomb gaps are much larger than the effective transfer parameter and therefore the transfer processes act within the low lying Coulomb manifold ${ }^{1} A_{1},{ }^{1} E,{ }^{1} T_{2},{ }^{3} T_{1},{ }^{3} T_{2}$ (pair $\mathbf{I}$ ).

Within this approximation one can get a simple visual picture in which the levels of the system linearly depend on the characteristic ratio of parameters $P$ and $t$ (Figure 17). One can see that the ground state of the system is either spin singlet ${ }^{1} A_{1}$, or a mixture of spin triplet and spin-singlet levels ${ }^{1} E,{ }^{3} T_{1}$ (Figure 17). The former case explains the experimentally obseved spin pairing, which was also confirmed by the $a b$ initio evaluation of the transfer parameters [70].

\subsubsection{Vibronic problem in 2e-reduced Keggin anion: formulation}

The electronic levels evaluated in previous sections are related to the molecule with the fixed positions of the ions. This ensures a symmetric geometry of the system which, under this condition, has minimal total energy. At the same time in MV compounds the deformation of the structure produced by the extra electron is, in general, not small and cannot be 
neglected giving rise to the vibronic effects. It should be emphasised that the due account for the vibronic effects is not only a question of the quantitative accuracy, but rather a prerequisite for the qualitative description of the physical phenomenon of the self-trapping of the extra electron and the degree of localisation. The extent of this effect is described by the well-known Robin and Day classification of MV compounds according to the degree of localisation based on the consideration of the vibronic coupling. According with this classification, the MV Keggin anion belongs to Class II [71] (see also review article [43a]), thus exhibiting an intermediate vibronic coupling. The vibronic interaction in MV compounds can be described in the framework of generally accepted PKS vibronic model [71c]. Although this model is rather simplified, it successfully describes the key features of MV systems, such as the occurrence of a potential barrier between localised configurations. In particular, this model underlies the Robin and Day classification of MV compounds accordingly to degree of localisation.

The Hamiltonian of the JT (or in general, pseudo JT) system can be represented as

$$
H=H_{e}+\sum_{i} \frac{\hbar \omega_{i}}{2}\left(Q_{i}^{2}-\frac{\partial^{2}}{\partial Q_{i}^{2}}\right)+\sum_{i} v_{i} \boldsymbol{O}_{i} Q_{i} \equiv H_{e}+H_{o s c}+H_{e v}
$$

The electronic subsystem is described by the Hamiltonian $H_{e}$, whose eigenvalues (electronic levels) for the Keggin anion have been considered in Section 3.2.2. The second term $\left(H_{\text {osc }}\right)$ in Equation (23) is the harmonic oscillator Hamiltonian in which $Q_{i}$ are the dimensionless vibrational coordinates, $\omega_{\mathrm{i}}$ are the vibrational frequencies. Within the PKS model the vibronic coupling parameters $v_{i}$ and frequencies $\omega_{i}$ for all redox sites are assumed to be equal and hereunder will be denoted as $v$ and $\omega$. In the basic case of a MV dimer (for example, family of Creutz-Taube bi-ruthenium complexes [72]) the two molecular states of the opposite parity (bonding and antibonding) are mixed through the only antisymmetric vibration composed of the local 'breathing' vibrations. Finally, the last term in Equation (23) describes the vibronic coupling and summation is extended over PKS vibrations. In this term $\boldsymbol{O}_{i}$ are the matrices of the vibronic coupling (see Ref. [43a]).

Very often the description of the properties of MV complexes is based on the adiabatic approximation, in particular, the Robin and Day classification employs the adiabatic picture. This approximation assumes that the electronic motion is much faster than the nuclear one. Consequently, within the adiabatic approximation one can neglect the kinetic energy of the ions (second term in the Hamiltonian $H_{o s c}$ ) and, therefore, the energy levels of the system can be associated with the full potential energy which includes also the electronic energy as function of the vibrational coordinates (adiabatic potential). Although the adiabatic approximation is very spectacular and gives a good qualitative description, it has a rather limited frame of the applicability. This was realised in the basic works dealing with the vibronic coupling in MV compounds where the dynamic nature of the vibronic problem has been underlined [71], especially for the evaluation of the shape-functions of the intervalence optical absorption bands. Actually, in the anti-crossing region of the adiabatic potentials the system performs fast Landau-Zener transitions so that the nuclear motion cannot be separated from the electronic one and can not be admitted as slow. In this view it is worth to mention that the theory of MV systems and metal complexes, which can be referred to as 'chemical theory beyond the Born-Oppenheimer paradigm', has been developed (see book [73]). 


\subsubsection{Symmetry assisted approach to the multidimensional non-adiabatic Jahn-Teller problem}

To get the dynamic (quantum-mechanical) solution of the vibronic problem of mixedvalency, one should find the eigenvalues and eigen-vectors of the Hamiltonian, Equation (23); this includes, along with the potential energy, the kinetic energy of nuclear motion. The basis functions which can be used to construct the matrix Hamiltonian and to solve the dynamic problem are represented by the products of the electronic wave-functions (eigenvectors of the Hamiltonian $H_{e}$ ) and harmonic oscillator functions (eigenvectors of the Hamiltonian $H_{o s c}$ ). Since the harmonic oscillator has the infinite number of the levels, the basis for solving the quantum-mechanical problem is also infinite. In the case of degenerate vibrations, which is peculiar for the JT and pseudo JT situations, the levels are multiple degenerate and this brings additional serious difficulties. Of course, the infinite matrices must be truncated but the convergence of the procedure is slow, so that one needs a more efficient symmetry adapted approach to the dynamic vibronic problem. Let us illustrate such recently developed approach by considering as an example the MV 2e-reduced Keggin anion.

The full electronic basis of the Keggin anion can be truncated while solving the vibronic problem. It is worthwhile to include in the electronic basis only the six low-lying electronic states corresponding to the pair I (Figures 15 and 16), that is, the states ${ }^{1} A_{1},{ }^{1} E,{ }^{1} T_{2}$ for $S=0$ and ${ }^{3} T_{1},{ }^{3} T_{2}$ for $S=1$. Such truncation of the electronic basis is acceptable under the condition that the effective energy of the vibronic coupling is smaller than the mean gap between the levels of the pairs I and II. This condition seems to be quite realistic for the 2e-reduced Keggin anion. Then one should introduce the symmetry adapted PKS vibrations for the Keggin structure involving twelve sites. The analysis of the PKS model for the Keggin anion shows [68b] that two kinds of vibrations are active, namely the doubly degenerate e- vibrations and triply degenerate $t_{2}$-vibrations. Pictorial representation of the PKS coordinates in terms of 'breathing' displacements of the octahedral metal sites is given in Figure 18.

The vibronic coupling leads to a rather complicated JT/pseudo JT problems involving six-dimensional electronic space and five-dimensional vibrational one. In the standard notation the problem can be designated as $\left({ }^{1} T_{2}+{ }^{1} E+{ }^{1} A_{1}\right) \otimes\left(e+t_{2}\right)$ and $\left({ }^{3} T_{1}+{ }^{3} T_{2}\right) \otimes\left(e+t_{2}\right)$, which means that the vibronic coupling with five vibrations $(e$ and $t_{2}$ ) mixes six electronic states. The frequencies of $T_{2}$ and $E$ vibrations coincide with the frequency of the local 'breathing' mode and, therefore, the electronic system is effectively coupled to a five-dimensional oscillator whose unperturbed levels are enumerated by the quantum number $n=n_{1}+n_{2}+n_{3}+n_{4}+n_{5}$. These levels are multiple degenerate because a definite number $n$ can arise from different combinations of $n_{i}$. Thus, the dimension of the vibrational space is $6 G(N)$, where the factor 6 appears as the dimension of the electronic subspace, while $G(N)$ is the dimension of the vibrational subspace including five modes $\left(e\right.$ and $t_{2}$ ). Simple calculations show that $6 G(N)$ can be expressed as

$$
6 G(N)=6 \sum_{n=0}^{N} g(n)=\frac{1}{20}(N+1)(N+2)(N+3)(N+4)(N+5),
$$

where $g(n)=\frac{1}{24}(n+1)(n+2)(n+3)(n+4)$ is the total degeneracy of the level with a given $n$, while $N$ is the number of the levels included in the basis. One can see that the size 


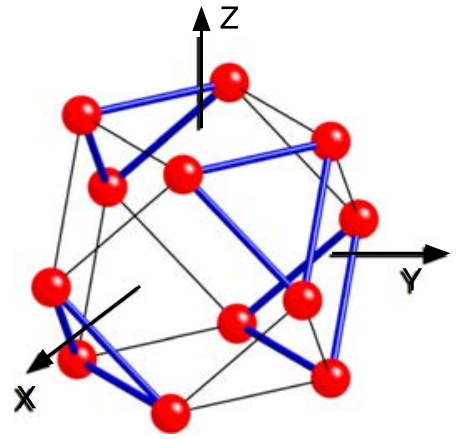

all $Q_{\Gamma \gamma}=0$

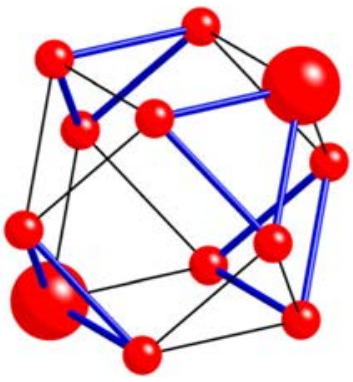

$Q_{E u}>0$

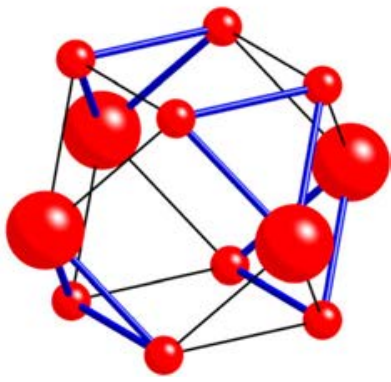

$Q_{T_{2} \xi}>0$

Figure 18. Pictorial representation of the shapes of the $E$ - and $T_{2}$ - types of PKS vibrations in Keggin anion 'breathing' displacements of the coordination surrounding of the metal sites. Red balls symbolise the octahedral units. Left part of the figure shows symmetric (undistorted, $\mathbf{T}_{d}$ ) structure. Large and small circles symbolise expanded and compressed octahedral sites, intermediate size circles denote undistorted units. One component for each type (irrep) is shown. Colour online.

Table 2. Group-theoretical classification of five low lying vibrational levels for PKS vibrations of $e$ - and $t_{2}$-types in Keggin anion with $\mathbf{T}_{\mathrm{d}}$ point symmetry.

\begin{tabular}{ccc}
\hline$n$ & Vibrationse & Vibrations $t_{2}$ \\
\hline 0 & $A_{1}$ & $A_{1}$ \\
1 & $E$ & $T_{2}$ \\
2 & $A_{1}+E$ & $A_{1}+E+T_{2}$ \\
3 & $A_{1}+A_{2}+E$ & $A_{1}+T_{1}+2 T_{2}$ \\
4 & $A_{1}+2 E$ & $2 A_{1}+2 E+T_{1}+2 T_{2}$ \\
\hline
\end{tabular}

of the matrix of the vibronic Hamiltonian becomes huge even if a relatively small number of the vibrational levels is included in the basis set employed to solve the combined JT/pseudo JT problems $\left({ }^{1} T_{2}+{ }^{1} E+{ }^{1} A_{1}\right) \otimes\left(e+t_{2}\right)$ and $\left({ }^{3} T_{1}+{ }^{3} T_{2}\right) \otimes\left(e+t_{2}\right)$ for the pair $\mathbf{I}$ in the $2 \mathrm{e}-$ reduced Keggin anion. For example, for $N=40$ the size of the matrix of to be diagonalised is $6 G(N)=7.330 .554$. This demonstrates that, in order to make the problem solvable, an efficient use of the symmetry is needed.

At the first step it is worthwhile to reveal the symmetry labels for the harmonic oscillator states for the two involved degenerate vibrations. Omitting the details of the grouptheoretical procedure (see Ref. [43]), we give the final results in Table 2. One can see that the ground state is non-degenerate for both types of vibrations. The first level $(n=1)$ is a doublet $E$ for $e$-vibrations and triplet $T_{2}$ for $t_{2}$-vibrations that means that the first vibrational level of the system is 6 -fold degenerate. Then the $n=2$ level involves irreps $A_{1}+E$ for $e$-vibrations and $A_{1}+E+T_{2}$ for $t_{2}$-vibrations. The results for $n=0,2,3,4$ are given in Table 2. 
Table 3. Symmetry adapted wave-functions of a bi-dimensional harmonic oscillator (e-vibrations) expressed in terms of simple functions with certain occupation numbers $\left|n_{1} n_{2}\right\rangle,\left(n=n_{1}+n_{2}\right)$.

\begin{tabular}{ll}
\hline$n=2$ & \multicolumn{1}{c}{$n=3$} \\
\hline$\left|2, A_{1}\right\rangle=\frac{1}{\sqrt{2}}(|20\rangle+|02\rangle)$, & $\left|3, A_{1}\right\rangle=\frac{1}{2}(\sqrt{3}|21\rangle-|03\rangle)$ \\
$|2, E x\rangle=|11\rangle$ & $\left|3, A_{2}\right\rangle=\frac{1}{2}(|30\rangle-\sqrt{3}|12\rangle)$ \\
$\left.|2, E y\rangle=\frac{1}{\sqrt{2}}(|20\rangle-|02\rangle)\right\} E$ & $|3, E x\rangle=\frac{1}{2}(\sqrt{3}|30\rangle+|12\rangle)$ \\
& $\left.|3, E y\rangle=\frac{1}{2}(|21\rangle+\sqrt{3}|03\rangle)\right\} E$ \\
\hline
\end{tabular}

Notes: Short notations; $\left|n=2, A_{1}\right\rangle \equiv\left|2, A_{1}\right\rangle$, etc. are used.

To simplify the solution of the eigen-problem one should construct the functions belonging to all allowed irreps for each value of $n=n_{1}+n_{2}+n_{3}+n_{4}+n_{5}$. The procedure includes the design of the operators (which we proposed to refer to as 'multivibronic operators' [43]), which being applied to the degenerate harmonic oscillator functions $\left|n_{1} n_{2} n_{3} n_{4} n_{5}\right\rangle$, create the vibrational states belonging to the allowed irreps of the point symmetry group. Then, the symmetry adapted vibrational functions are coupled to the electronic ones to give full vibronic functions of certain symmetry (irreps). The key result is that the full matrix of the Hamiltonian to be diagonalised can be reduced to reach block-structure, with each block corresponding to a definite irrep. The mathematical tools to solve this problem are described in detail in Ref. [43]. With the aim to illustrate the results, we give here the final expressions for the vibrational functions (which are normalised and orthogonal) for the double degenerate vibrations of the e-type.

The functions of the bi-dimensional oscillator can be labelled as $\left|n_{1} n_{2}\right\rangle$ with $n_{1}=$ $0,1 \ldots \infty, n_{2}=0,1 \ldots \infty$. The basis of the irrep $E$ in $\mathbf{T}_{\mathrm{d}}$ is chosen in a standard way: $u \propto 3 z^{2}-r^{2}, v \propto \sqrt{3}\left(x^{2}-y^{2}\right)$. One can find the symmetry adapted basis in terms of the linear combinations of simple vibrational functions $\left|n_{1} n_{2}\right\rangle$ with the definite occupation numbers. The final results for the vibrational levels with $n=2$ and $n=3$ are given in Table 3.

In a similar way one can obtain the functions for $n=4$, etc. and those for the $t_{2}$ vibrations. Finally, these vibrational functions are coupled to the electronic ones. This procedure represents a core of the symmetry assisted approach to the vibronic problem. The efficiency of the symmetry assisted approach is illustrated in Table 4 containing the dimensions of the electron-vibrational spaces for different blocks arising in the dynamic JT/pseudo JT problems $\left({ }^{1} T_{2}+{ }^{1} E+{ }^{1} A_{1}\right) \otimes\left(e+t_{2}\right)$ and $\left({ }^{3} T_{1}+{ }^{3} T_{2}\right) \otimes\left(e+t_{2}\right)$ in Keggin anion for different dimensions of the truncated basis. One can see, for example, that for $N=12$ the full dimension of the Hilbert spaces for spin-singlets and spin-triplets is 37.128. The use of the symmetry adapted basis essentially simplifies the computational procedure by blocking the full matrix. Thus, for $N=12$ the maximum sizes of the blocks are $4.676 \times 4.676$ (for each of the terms ${ }^{1} T_{2},{ }^{3} T_{1}$ and ${ }^{3} T_{2}$ ).

In Ref. [43d] we have presented a program called VIBPACK, based on the symmetry assisted approach, which takes full advantage of the point symmetry arguments. The VIBPACK program allows to tackle the multidimensional dynamic JT/pseudo JT problems 
Table 4. Dimensions of the electron-vibrational spaces for the blocks arising for the JT/pseudo JT problems for the pair I in Keggin anion.

\begin{tabular}{lcccccc}
\hline$N$ & $6 G(N)$ & $A_{1}$ & $A_{2}$ & $E$ & $T_{1}$ & $T_{2}$ \\
\hline Spin singlets & & & & & & \\
10 & 18.018 & 819 & 728 & 1.547 & 2.184 & 2.275 \\
11 & 26.208 & 1.176 & 1.065 & 2.240 & 3.192 & 3.304 \\
12 & 37.128 & 1.652 & 1.512 & 3.164 & 4.536 & 4.676 \\
Spin triplets & & & & & & \\
10 & 18.018 & 728 & 728 & 1.456 & 2.275 & 2.275 \\
11 & 26.208 & 1.064 & 1.064 & 2.128 & 3.304 & 3.304 \\
12 & 37.128 & 1.512 & 1.512 & 3.024 & 4.676 & 4.676 \\
\hline
\end{tabular}

through the reduction of the vibronic matrices to full extent. Using VIBPACK program one can calculate the energy levels of large scale vibronic systems and related thermodynamic and spectroscopic characteristics, such as magnetic moments and vibronically assisted optical bands. The last requires large vibronic basis and therefore serves as a benchmark of the accuracy of the VIBPACK program.

Figure 19 shows a sample of the vibronic levels of the MV Keggin anion calculated as functions of the vibronic coupling parameter $v$ for a rather restricted basis set $N=8$. For the sake of simplicity only the pair $\mathbf{I}$ is considered. At $P=0$ one obtains the electronic levels superimposed with the unperturbed harmonic oscillator levels. The level ${ }^{1} A_{1}$, which is the ground state of spin paired system, is chosen as a reference level for both spinsinglets and spin-triplets. Then, one can see that a simple equidistant picture of the levels is strongly distorted when the vibronic coupling increases. Finally, when the vibronic coupling is strong enough, the excited levels approach the ground level which can be attributed to the presence of degeneracy due to the existence of several energetically equivalent deep wells of the adiabatic potential or to Ham reduction of the transfer processes. The analysis of the composition of the vibronic spin-levels demonstrates the effective increase of the magnetic moment of the system that can be considered as a result of the combined action of the JT/pseudo JT couplings.

\section{Mixed-valence reduced polyoxometalates with partial electron delocalisation}

\subsection{The polyoxovanadate anion $\left[\mathrm{KV}_{4}^{I V} V_{8}^{V}\left(\mathrm{O}_{3} \mathrm{AsOH}\right)_{3} \mathrm{O}_{27}\left(\mathrm{AsO}_{4}\right)\right]^{6-}$}

\subsubsection{Structure of polyoxovanadate anion $\left[\mathrm{KV}_{4}^{I V} V_{8}^{V}\left(\mathrm{O}_{3} \mathrm{AsOH}\right)_{3} \mathrm{O}_{27}\left(\mathrm{AsO}_{4}\right)\right]^{6-}$}

The systems with partial electron delocalisation contain interacting spin-localised and delocalised moieties in the same molecule. The $\left[\mathrm{KV}_{4}^{\mathrm{IV}} \mathrm{V}_{8}^{\mathrm{V}}\left(\mathrm{O}_{3} \mathrm{AsOH}\right)_{3} \mathrm{O}_{27}\left(\mathrm{AsO}_{4}\right)\right]^{6-}$ cluster anion belongs to a special class of MV POMs containing coupled localised and delocalised triangular subunits which form the magnetic part of the molecule. Ball-and-stick representation of this cluster, which will be referred to as $\mathrm{V}^{\mathrm{IV}}{ }_{4} \mathrm{~V}_{8}$, is shown in Figure 20. Further details of the structure can be found in Ref. [74]. The magnetic properties of $\mathrm{V}^{\mathrm{IV}}{ }_{4} \mathrm{~V}^{\mathrm{V}}$ are interrelated with the metal sites, which involve a localised trimeric $\mathrm{V}_{3}^{4+}$ unit (with three spins 1/2) and a MV V IV $\mathrm{V}_{2}$ unit comprising one unpaired electron shared among three sites [74]. Therefore, this system can be referred to as a system with partial electron 
(a)

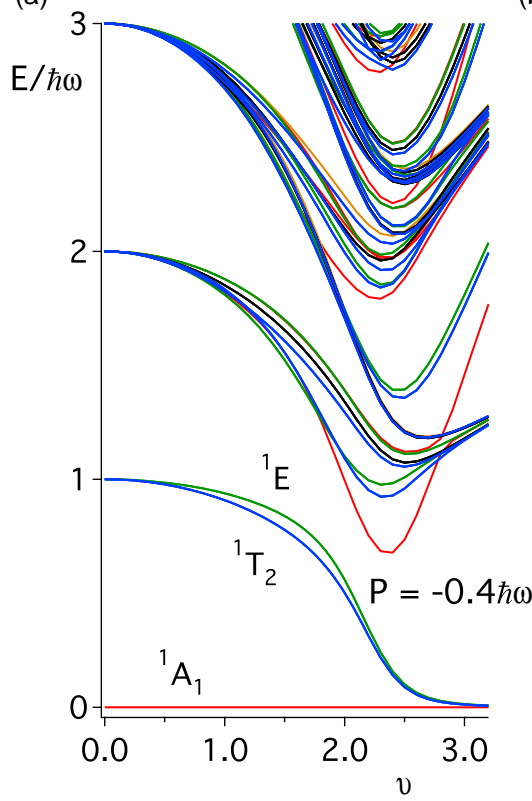

(b)

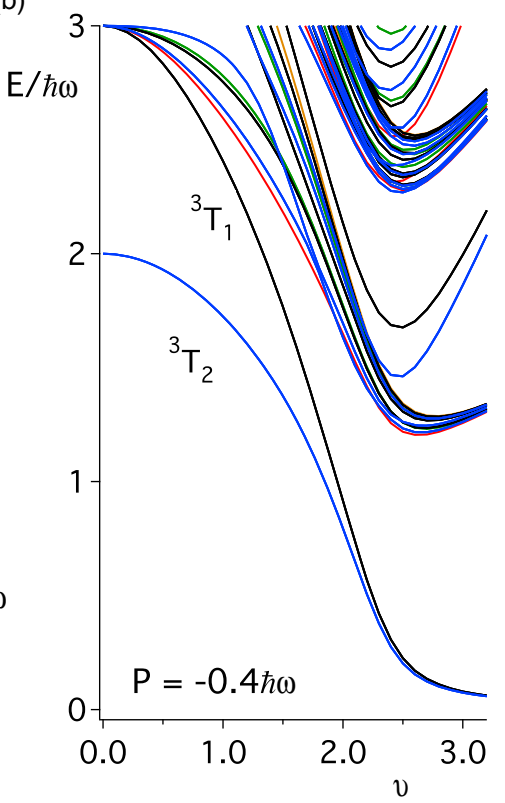

Figure 19. Vibronic levels vs vibronic coupling parameter for spin-singlet (left) and spin-triplets (right) terms belonging to the ground manifold (pair I) of the 2e-reduced Keggin anion. The ground level $\left({ }^{1} A_{1}\right)$ is taken as the origin in both parts of the energy pattern. The energies are evaluated using the VIBPACK program.

delocalisation in which the localised and itinerant electrons coexist and interact. The network of the magnetic sites in the structure of $\mathrm{K}_{6}\left[\mathrm{H}_{3} \mathrm{KV}_{12} \mathrm{As}_{3} \mathrm{O}_{39}\left(\mathrm{AsO}_{4}\right) \cdot 8 \mathrm{H}_{2} \mathrm{O}\right]$ is shown in Figure 21a. Exchange coupled (spin-localised) trimer $d^{1}-d^{1}-d^{1}$ (sites $\left.a, b, c\right)$ and spindelocalised $d^{1}-d^{0}-d^{0}$-cluster ( $\operatorname{sites} \alpha, \beta, \gamma$ ) lie in parallel planes, so that the overall symmetry is $\mathbf{C}_{3 \mathrm{v}}$ as well as the 'site symmetry' of each subunit.

\subsubsection{Group-theoretical classification of the delocalised states in mixed-valence systems}

The group-theoretical classification for partially localised systems has some peculiarities which will be described below. For highly symmetric systems with partial delocalisation the symmetry assignation can be done by combining the states of the two subunits, localised and delocalised. The exchange coupled (localised) spin systems have been considered in Section 2.1.4. Let us illustrate the procedure for the spin-delocalised subsystem by taking as an example a trimeric cluster $d^{1}-d^{1}-d^{2}$ with one electron delocalised over three oneelectron spin cores.

Hund type (ferromagnetic) coupling of a spin core with a trapped electron forms a $S^{*}=1$ centre, while the remaining centres have spins $1 / 2$. The spin coupling scheme for this system can be symbolised as

$$
3\left(D^{(1 / 2)} \otimes D^{(1 / 2)} \otimes D^{(1)}\right)=3 D^{(0)}+6 D^{(1)}+3 D^{(2)},
$$


(a)

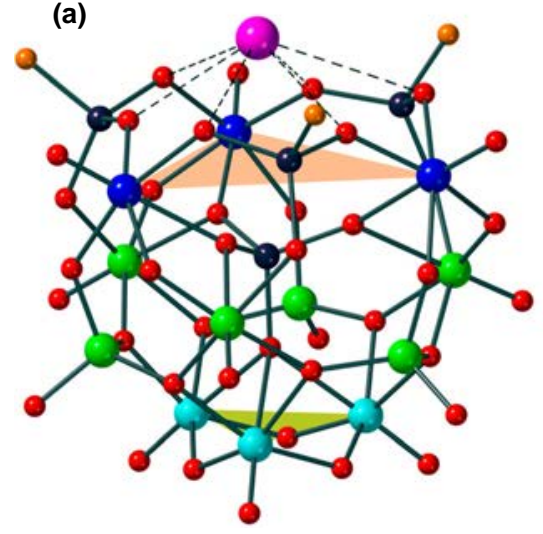

(b)

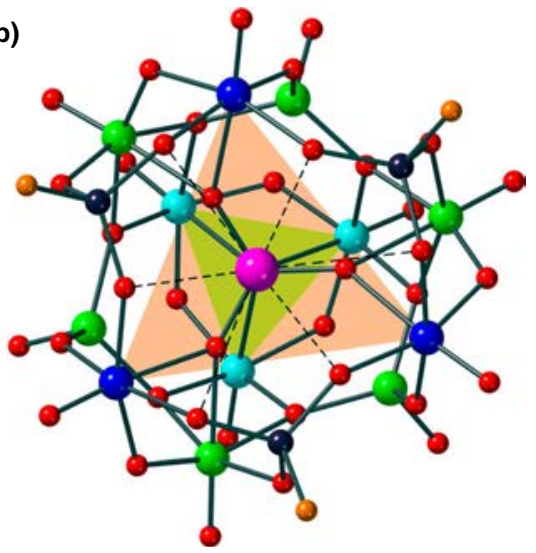

Figure 20. Ball-and-stick representation of the cluster anion $\left[\mathrm{KV}_{4}^{\mathrm{IV}} \mathrm{V}_{8}^{\mathrm{V}}\left(\mathrm{O}_{3} \mathrm{AsOH}\right)_{3} \mathrm{O}_{27}\left(\mathrm{AsO}_{4}\right)\right]^{6-}$. General view of the structure indicating localised frustrated triangle of $\mathrm{V}^{\mathrm{IV}}$ ions (shaded by orange) and delocalised triangle (green) (a); view from the direction along $C_{3}$ axis (b).
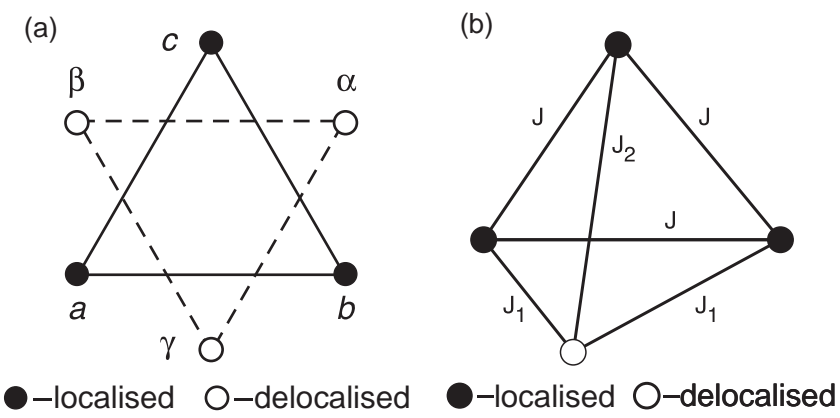

Figure 21. Scheme of the magnetic sites in a double triangular vanadium cluster in $\mathrm{V}^{\mathrm{IV}}{ }_{4} \mathrm{~V}_{8}{ }_{8}(\mathrm{a})$ and scheme of the exchange pathways in a localised configuration (b).

where the factor 3 on the left-hand side of Equation (25) takes into account three possible localizations and two possible spin-triplet states which appear due to two possible intermediate spins in three-spin coupling scheme.

The full basis set involves four-electron determinants $\left|a_{m_{a}}\left(s_{a}\right) b_{m_{b}}\left(s_{b}\right) c_{m_{c}}\left(s_{c}\right)\right|$ related to three possible localizations of the 'extra' electron. For example, for $c$-localisation $s_{a}=s_{b}=$ $1 / 2$ and $s_{c}=1$. For $M=S=2$, there is only a determinant for each localisation and we find a three-dimensional representation with the basis set:

$$
\left|a_{1 / 2}\left(\frac{1}{2}\right) b_{1 / 2}\left(\frac{1}{2}\right) c_{1}(1)\right|,\left|a_{1 / 2}\left(\frac{1}{2}\right) b_{1}(1) c_{1 / 2}\left(\frac{1}{2}\right)\right|,\left|a_{1}(1) b_{1 / 2}\left(\frac{1}{2}\right) c_{1}(1)\right|
$$

for which one gets $\chi^{(M=2)}(\hat{R})=\chi^{(S=2)}(\hat{R})$ for all $\hat{R}$ and then we find $3 D^{(2)} \rightarrow{ }^{5} A_{2}+{ }^{5} E$. Continuing this procedure as in previous example one can find the following result for the 
group-theoretical classification of the delocalised states of the $d^{1}-d^{1}-d^{2}$ system:

$$
\begin{aligned}
& 3 D^{(2)} \rightarrow{ }^{5} A_{2}+{ }^{5} E, \\
& 6 D^{(1)} \rightarrow{ }^{3} A_{1}+{ }^{3} A_{2}+2{ }^{3} E, \\
& 3 D^{(0)} \rightarrow{ }^{1} A_{1}+{ }^{1} E .
\end{aligned}
$$

One can see that the only $2 \times 2$ matrix corresponding to $2^{3} E$ should be diagonalised while the remaining energies can be found directly as the diagonal matrix elements of the Hamiltonian in the symmetry adapted bases.

\subsubsection{Group-theoretical classification of spin multiplets in a partially delocalised system, the main features of $V_{4}^{I V} V_{8}^{V}$}

To make some general qualitative judgments one has to classify the states of the system from the symmetry point of view. The basis set is formed by $3 \cdot 2^{4}=48$ determinants $\left|a_{m_{a}}\left(\frac{1}{2}\right) b_{m_{b}}\left(\frac{1}{2}\right) c_{m_{c}}\left(\frac{1}{2}\right) \alpha_{m_{\alpha}}\left(\frac{1}{2}\right)\right|$, etc., which are built from the orbitals localised on the sites depicted in Figure 22 (notations of spin-orbitals are the same as in previous sections). Applying operations of $\mathbf{C}_{3 \mathrm{v}}$ symmetry group and using the approach so far discussed (we omit here the details), one can find the $S \Gamma$-multiplets of the system with partial delocalisation. For example, for maximal $M=S=2$ we have the three-dimensional basis which consists of the four-electron determinants:

$$
\left|a_{1 / 2} b_{1 / 2} c_{1 / 2} \alpha_{1 / 2}\right|,\left|a_{1 / 2} b_{1 / 2} c_{1 / 2} \beta_{1 / 2}\right|,\left|a_{1 / 2} b_{1 / 2} c_{1 / 2} \gamma_{1 / 2}\right|
$$

with $\Gamma^{(M=2)}=\Gamma^{(S=2)}=A_{2}+E$, and hence, we find ${ }^{5} A_{2}+{ }^{5} E$ multiplets. Then, in the present case when the subunits have common $C_{3}$ axis, the $S \Gamma$-multiplets of the partially delocalised system can be found as the decomposition of the direct products: $D^{\left(S_{e x c}\right)} \otimes$ $D^{\left(S_{M V}\right)}$ and $\Gamma_{e x c} \times \Gamma_{M V}$, where $S_{e x c} \Gamma_{e x c}$ and $S_{M V} \Gamma_{M V}$ are the terms of the localised and delocalised units (the Pauli principle in this case does restrict possible resulting states). Finally, one can find the following group-theoretical assignment:

$$
\left({ }^{4} A_{2}+{ }^{4} E\right) \times\left({ }^{2} A_{1}+{ }^{2} E\right)={ }^{1} A_{1}+{ }^{1} A_{2}+{ }^{1} E+{ }^{3} A_{1}+2{ }^{3} A_{2}+2{ }^{3} E+{ }^{5} A_{2}+{ }^{5} E .
$$

One can see that the 48-dimensional space is reduced to the blocks of lower dimensionality, i.e. into the subspaces with the maximal dimension of 2 that allows to find the analytical solutions of the eigen-problem in this rather complicated system.

The theoretical model [74] includes the following key interactions which are specific for a partially delocalised system: HDVV exchange in the three localised configurations, AS exchange in the localised spin-frustrated triangle (see Section 2.1) and electron delocalisation over three sites in the delocalised triangle. The analysis of these interactions along with the symmetry of the states shows that they are in a complicated interplay leading to interesting physical conclusions about the magnetic behaviour of $\mathrm{V}_{4}^{\mathrm{IV}} \mathrm{V}_{8}^{\mathrm{V}}$. A brief conclusion is that in the localised limit (see Figure 21b) the AS exchange is partially suppressed by the low symmetry component of the isotropic exchange produced by the inter-triangle coupling. When the delocalisation is involved, it can restore the trigonal (actually, axial) symmetry and the AS exchange appears again as a first order effect in the orbital doublets, while the orbital singlets are not involved in this phenomenon. Thus, the coexistence of the localised and delocalised states is an immanent feature of the partially delocalised systems. 


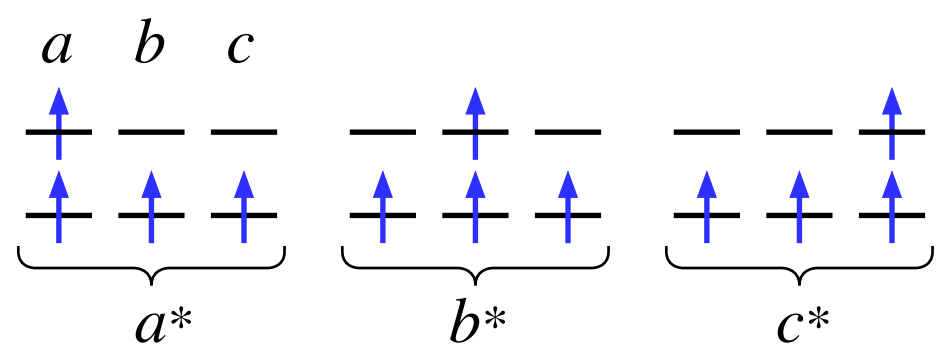

Figure 22. Scheme of the one-electron levels of the MV trimer of $d^{1}-d^{1}-d^{2}$ in the three possible localizations of the itinerant electron.

This can be understood in terms of the so called motional narrowing, which in the case under consideration can be referred to as averaging of the isotropic inter-triangle exchange interactions which drastically increases the magnetic anisotropy of the system (see details in Ref. [74]).

\subsection{Partially delocalised cluster anion $\left[V_{8}^{I V} V_{4}^{V} A s_{8} \mathrm{O}_{40}\left(\mathrm{H}_{2} \mathrm{O}\right)\right]^{4-} \equiv\left\{V_{12} A s_{8}\right\}$}

Two more complicated isostructural dodecanuclear systems $\left[\mathrm{V}_{12} \mathrm{As}_{8} \mathrm{O}_{40}\left(\mathrm{HCO}_{2}\right)\right]^{3-}$ (I) and $\left[\mathrm{V}_{12} \mathrm{As}_{8} \mathrm{O}_{40}\left(\mathrm{HCO}_{2}\right)\right]^{5-}$ (II) (Figure 23) with partial electron delocalisation have been considered in Ref. [75]. These compounds have been modelled under the assumption that they contain a central $\mathrm{V}_{4}^{4+}$ square containing four localised spins sandwiched between two spin-delocalised squares containing one (in I) or two (in II) delocalised electrons. The theoretical consideration of these rather complicated POMs is quite similar to that described in Section 4.1. The model for the partially delocalised $\left\{\mathrm{V}_{12} \mathrm{As}_{8}\right\}$ systems includes Coulomb interaction between itinerant electrons in different localised configurations, delocalisation effects and exchange interactions between spins located at different squares.

This rather complicated problem can be solved with the aid of symmetry arguments, which also help to understand the results in terms of the physical concepts. The methodology is quite similar to that so far described. At the first step we consider the exchange problem for the fully localised $s=1 / 2$ square, which has an obvious analytical solution. Then, one can assign the labels of the irreps of the point group $\mathbf{D}_{4 \mathrm{~h}}$ to the spin levels of the internal square (using the approach described in Section 2.1.4). Using the method of the group-theoretical classification for MV systems (Section 4.1.2) one can find at the second step the labels of the terms for the MV squares containing two-electrons. The localised and delocalised subunits have a common $\mathrm{C}_{4}$ axis which allows to combine the terms of three subunits in order to find terms of the entire system and the energy pattern. The electronic and vibronic problems for the external MV bi-electronic squares are considered in detail in [76] An interesting feature for this kind of system is the spin polarisation effect exerted by the moving electrons over the localised square subunit. This effect tends to stabilise an electron configuration in which the upper electron pair is inverted with respect to the lower one. This feature may have a promising application of this cluster as a quantum cellular automata (QCA). 

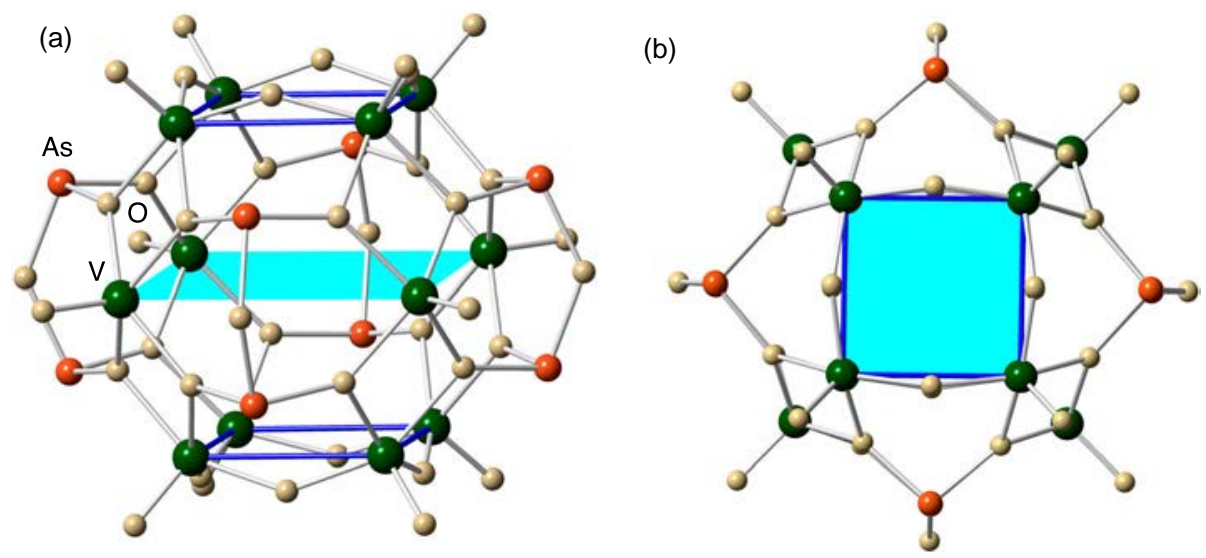

Figure 23. Ball-and-stick representation of the cluster anion $\left[\mathrm{VV}_{8} \mathrm{VV}_{4} \mathrm{As}_{8} \mathrm{O}_{40}\left(\mathrm{H}_{2} \mathrm{O}\right)\right]^{4-} \equiv\left\{\mathrm{V}_{12} \mathrm{As}_{8}\right\}$ from two perspectives emphasising the central $V^{\mathrm{IV}}{ }_{4}$ rectangle (blue) which is sandwiched between two mixed-valent $\mathrm{V}^{\mathrm{V}}{ }_{2} \mathrm{~V}_{2}$ groups (blue lines squares). Hydrogen positions of the enclosed water molecule are omitted for clarity (V-green; As-orange, O-beige). Colour online.

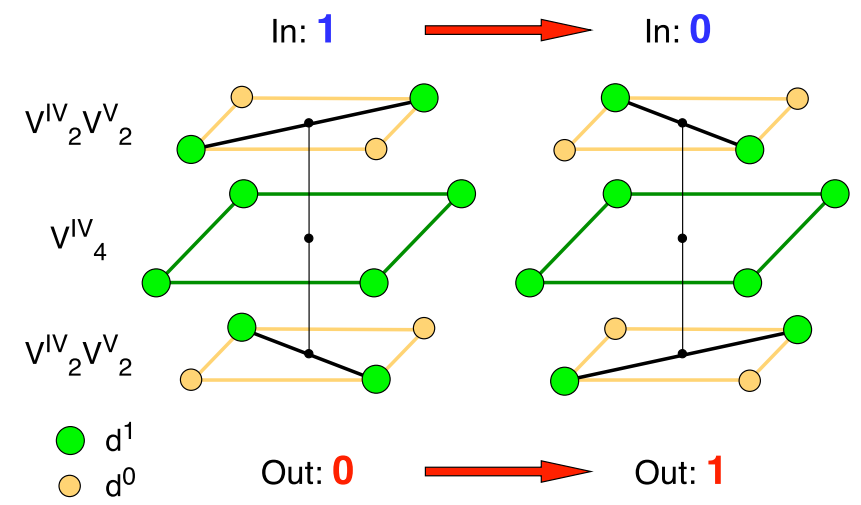

Figure 24. Scheme of the function of the $\left\{\mathrm{V}_{12} \mathrm{As}_{8}\right\}$ POM as quantum inverter. Only the two external squares in MV metal network are shown (balls). Colouring: occupied sites-green balls, empty sites-yellow balls. Colour online.

Thus, as distinguished from the initial idea of the molecular QCA, in which a molecule was supposed to act as a quantum cell, the MV cluster $\mathrm{V}_{12} \mathrm{As}_{8}$ can act as a logical gate, namely as a quantum inverter. This is illustrated by Figure 24 in which the switching function is shown. The upper square is assumed to play a role of the input cell which is set to the binary 1 . As one can see (Figure 24) the mobile pair of the electrons in the lower rectangle tends to be localised in such a way to encode the binary digit $\mathbf{0}$ in order to gain the intercell Coulomb repulsion energy. The lower square can be referred to as output cell and therefore this POM is expected to act as the quantum inverter. This qualitative consideration should be supported by quantitative estimations in order to justify the real possibility to design the quantum inverters based on this POM. 


\section{Conclusions and comments beyond the scope of this review}

In this article we have summarised the main symmetry based theoretical tools as applied to large magnetic clusters with complex molecular structures using complex POMs as model systems. The main emphasis is on the question of how the symmetry can help us to evaluate the physical characteristics and (mainly) to understand the properties of these molecular compounds in most general terms. The symmetry properties are not confined by the approximate models (which sometimes are hard to substantiate) and for this reason the symmetry based approaches are especially valuable to treat large POM clusters containing exchange coupled localised spins, fully delocalised electrons and, finally, combinations of localised spins and delocalised electrons.

We have discussed appropriate symmetry concepts for each of these three types of POMs, namely: (1) spin symmetry that is taken into account through application of the ITO approach. The use of ITO and related MAGPACK software allows to essentially reduce the matrices of the HDVV Hamiltonian and in this way to facilitate evaluation of the thermodynamic and spectroscopic properties of high-nuclearity metal clusters; (2) the group-theoretical classification (assignment) of the exchange multiplets, dealing with both spin-symmetry and point symmetry, which allows to establish one-to-one correspondence between spin quantum numbers in HDVV scheme and irreps of the point group of the exchange system. This theoretical tool essentially simplifies the analysis of the magnetically anisotropic contributions to the exchange Hamiltonian in general terms of symmetry and pseudo-angular momentum representation. Although the anisotropic contributions are small within the physical frame of the HDVV model, they can play crucial role in symmetric systems possessing degenerate multiplets. Also one can find the selection rules for the magnetic resonance, optical and inelastic neutron scattering transitions. This approach provides direct access to the selection rules for the active JT coupling and gives clear understanding of the interrelation between spin frustration and structural instabilities; (3) group-theoretical classification of the delocalised electronic and electron-vibrational states of MV compounds (including the states of delocalised electronic pair) in terms of spin and point symmetries that essentially reduces the time of calculations and gives access to the selection rules for different kinds of transitions. This becomes crucially important if one is aimed to solve dynamical JT vibronic problems (inherently related to mixed-valency), which are sometimes hardly executable even when strongly truncated basis sets are used. The proposed approach (realised as the MVPACK program) that includes the design of the symmetry adapted vibronic basis enormously extends computational abilities in such cases.

Although we have discussed the main symmetry related theoretical tools in molecular magnetism and a wide (even being intentionally restricted by POMs) range of applications, it is worth mentioning what was not said in this article. Here we have not considered a rather difficult (and requiring complex mathematical tools) problem of exchange interactions in polynuclear metal complexes involving orbitally degenerate metal ions that was a subject of the study in Ref. [77] (see details in review article [77a]). Under this condition the conventional HDVV model dealing with the spin degrees of freedom becomes inapplicable. In general, the degenerate magnetic ions carry an unquenched orbital angular 
momentum that manifests itself in all their magnetic properties. The exchange interaction between orbitally degenerate metal ions can be described by the so-called orbitallydependent exchange Hamiltonian deduced in Ref. [77]. The structure of this Hamiltonian is more complicated and involves both spin and orbital variables (the so-called orbital matrices). From the physical point of view the degeneracy of the constituent ions in clusters has shown to lead to an anomalously strong magnetic anisotropy that can be considered as the main physical manifestation of the unquenched orbital angular momentum in magnetic systems. An inherent interaction that should be taken into account under the condition of degeneracy (or pseudo degeneracy) is the spin-orbital coupling. The theoretical background was used to account for the magnetic properties of some magnetic clusters and low-dimensional systems such as binuclear face-shared unit $\left[\mathrm{Ti}_{2} \mathrm{Cl}_{9}\right]^{3-}$, the rare-earth compounds $\mathrm{Cs}_{3} \mathrm{Yb}_{2} \mathrm{Cl}_{9}$ and $\mathrm{Cs}_{3} \mathrm{Yb}_{2} \mathrm{Br}_{9}$, a zig-zag $\mathrm{Co}(\mathrm{II})$ chain exhibiting unusual combination of single-chain magnet behaviour and antiferromagnetic exchange coupling, a trigonal bypiramidal $\mathrm{Ni}_{3} \mathrm{Os}_{2}$ complex.

In some cases of orbital degeneracy a simplified approach can applied that takes into account orbital effect (such as spin-orbital coupling, orbital part of Zeeman intetaction, etc) but the exchange interaction is assumed to be isotropic. The systems of this type are exemplified by the $\mathrm{Fe}_{2} \mathrm{Co}$ trimers in heteronuclear iron-cobalt complex $\left[\mathrm{Fe}_{2} \mathrm{CoO}\left(\mathrm{CH}_{3} \mathrm{COO}\right)_{6}(3-\mathrm{Cl}-\mathrm{Py})_{3}\right]$ synthesised and characterised in Ref. [78]. This compound contains orbitally degenerate $\mathrm{Co}$ (II) ion in an octahedral position that possesses spin and also orbital angular momentum. In is to be noted that ITO technique allowed to include in the computational pipeline spins, orbital angular momentum, spin-orbital interaction and low-symmetry crystal field.

As a system involving two orbitally degenerate ions and exhibiting single molecule magnetism one has to mention the trigonal bipyramidal cyano-bridged cluster $\left.\left[\mathrm{Mn}^{\mathrm{III}}(\mathrm{CN})_{6}\right]_{2}\left[\mathrm{Mn}^{\mathrm{II}} \text { (tmphen) }\right)_{2}\right]_{3}$ (tmphen = 3, 4, 7, 8-tetramethyl-1, 10-phenanthroline) synthesised and experimentally characterised in Refs. [79,80]. Strong cubic field produced by six carbon ions leads to the ground orbital triplet ${ }^{3} T_{1}\left(t_{2}{ }^{4}\right)$ of each $\mathrm{Mn}$ (III) ion giving rise to a significant single ion anisotropy. These two examples illustrate also the universality of the approach based on ITO and corresponding software.

Finally, the problem of degeneracy (in mono- and polynuclear magnetic systems) is closely related to the Jahn-Teller or/and pseudo Jahn-Teller interactions. This problem is presently still has not received due attention in the theory of the magnetic clusters with constituent orbitally degenerate ions.

The applications discussed in this article clearly demonstrate the key role played by symmetry concepts for the understanding of the properties of magnetic clusters with complex structures and, in general, for the whole area of molecular magnetism. In this article we have summarised the applications of symmetry to the study of POMs in the framework of the effective Hamiltonian. This symmetry-adapted approach has shown to be very powerful to treat highly complex systems and to understand their fundamental physical properties. Still, this approach is parametric and in some cases needs to be complemented with an $a b$ initio evaluation of these local parameters (exchange, transfer, Coulomb repulsions), especially when the parametric approach has excessive flexibility or when the detailed inner nature of the parameters is important for the picture of 
the phenomenon under consideration. In this context, the results that illustrate an enormous progress achieved in the quantum-mechanical evaluation of electronic and vibronic parameters in molecular magnets can be found in Refs. [81,82].

\section{Disclosure statement}

No potential conflict of interest was reported by the author(s).

\section{Funding}

The present work has been supported by the EU [COST Action CA15128 Molecular Spintronics (MOLSPIN)]. The authors acknowledge support from the Ministry of Education and Science of Russian Federation [Agreement No.14.W03.31.0001-Institute of Problems of Chemical Physics of RAS, Chernogolovka]. J.M.C-J. and E.C. thank the Spanish MINECO [CTQ2017-89528-P, MAT201789993-R, Unit of Excellence María de Maeztu, MDM-20150538] and Generalitat Valenciana [Prometeo Programme of Excellence]. B.T. thanks University of Valencia for a Sabbatical Grant [Estades Temporals Investigadors Convidats].

\section{ORCID}

Boris Tsukerblat (i) http://orcid.org/0000-0002-8182-6140

Juan Modesto Clemente-Juan (1D http://orcid.org/0000-0002-3198-073X

Eugenio Coronado (iD http://orcid.org/0000-0002-1848-8791

\section{References}

[1] D. Gatteschi and R. Sessoli, Angew. Chem. Int. Ed. 42, 268 (2003). doi:10.1002/anie.200390099

[2] D. Gatteschi, R. Sessoli, and R.J. Villain, Molecular Nanomagnets (Oxford University Press, Oxford, 2006).

[3] A. Bencini and D. Gatteschi, Electron Paramagnetic Resonance of Exchange Coupled Systems (Springer, Berlin, 1990).

[4] C. Benelli and D. Gatteschi, Introduction to Molecular Magnetism: From Transition Metals to Lanthanides (Willey-VCH, Weinheim, 2015).

[5] (a) O. Kahn, Molecular Magnetism (VCH, New York, 1993) (b) J.-P. Launay, M. Verdaguer, Electrons in Molecules: From Basic Principles to Molecular Electronics (Oxford University Press, NY, 2018).

[6] Magneto-structural Correlation in Exchange Coupled Systems, edited by R. Willett, D. Gatteschi and O. Kahn, NATO ASI Series C140 (Kluwer, Dordrecht, 1985).

[7] (a) J. Miller and A. Epstein, MRS Bul. Nov. (2000) 21; (b) J.S. Miller and K.S. Min, Angew. Chem. Int. Ed., 48, 262-272 (2009).

[8] (a) G. Christou, D. Gatteschi, D.N. Hendrickson, and R. Sessoli, MRS Bull. 25, 66 (2000); (b) W. Wernsdorfer, N. Aliaga-Alcalde, D.N. Hendrickson, and G. Christou, Nature 416 (6879), 406-409 (2002); (c) A.J. Tasiopoulos, A. Vinslava, W. Wernsdorfer, K.A. Abboud, and G. Christou, Angew. Chem. Int. Ed. 43, 2117-2121 (2004); (d) R. Bagai and G. Christou, Chem. Soc. Rev. 38, 1011-1026 (2009); (e) G. Christou, Polyhedron, 24 (16-17), 2065-2075 (2005); (f) M. Soler, W. Wernsdorfer, K. Folting, M. Pink, and G. Christou, J. Am. Chem.Soc. 126 (7), 2156-2165 (2004); (g) S.J. Blundell, Contemp. Physics 48, 275-290 (2008); (h) J. Schnack, Contemp. Physics 60, 127-144 (2019); (i) A. Furrer and O. Waldmann. Rev. Mod. Phys. 85, 367-420 (2013).

[9] (a) R. Sessoli, H.-L. Tsai, A.R. Schake, S. Wang, J.B. Vincent, K. Folting, D. Gatteschi, G. Christou, and D.N. Hendrickson, J. Am. Chem. Soc. 115, 1804 (1993); (b) A.-L. Barra, D. Gatteschi, L. Pardi, A. Müller, and J. Döring, J. Am. Chem. Soc. 114, 8509 (1992). 
[10] R. Sessoli, D. Gatteschi, A. Caneschi, and M.A. Novak, Nature. 365, 141 (1993). doi:10.1038/365141a0

[11] (a) N. Casañ-Pastor, J. Bas-Serra, E. Coronado, G. Pourroy, and L.C.K. Baker, J. Am. Chem. Soc. 114, 10380 (1992); (b) E. Coronado and C.J. Gómez-García, Comments Inorg. Chem. 17, 255 (1995); (c) J.M. Clemente-Juan and E. Coronado, Coord. Chem. Rev. 193-195, 361-394 (1999); (d) A. Müller, F. Peters, M.T. Pope, and D. Gatteschi, Chem. Rev. 98, 239 (1998); (e) U. Kortz, A. Müller, J. van Slageren, J. Schnack, N.S. Dalal, and M. Dressel, Coord. Chem. Rev. 253, 2315-2327 (2009); (f) X. López, J.J. Carbó, C. Bo, and J.M. Poblet, Chem. Soc. Rev. 41, 7537-7571 (2012); (g) K.Y. Monakhov, W. Bensch, and P. Kögerler, Chem. Soc. Rev. 44, 8443-8483 (2015); (h) D.L. Long, R. Tsunashima, and L. Cronin, Angew. Chem, Int. Ed. 49, 1736-1758 (2010); (i) A. Proust, R. Thouvenot, and P. Gouzerh, Chem. Commun. 1837-1852 (2008); (j) E. Coronado, J.R. Galán-Mascarós, C.J. Gómez-García, and V. Laukhin, Nature 408, 447 (2000).

[12] (a) C.L. Hill, D.C. Duncan, and M.K. Harrup, Comments Inorg. Chem. 14, 367 (1993); (b) C.L. Hill and C. M. Prosser-McCartha, Coord. Chem. Rev. 143, 407 (1995); (c) R. Neumann, Prog. Inorg. Chem. 47, 317 (1998); (d) N. Mizuno and K. Yamaguchi, Chem. Rec. 6, 12-22 (2006); (e) A. Proust, R. Thouvenot, and P. Gouzerh, Chem. Commun. 1837-1852 (2008); (f) A. Müller, S. Talismanov, P. Kögerler, H. Bögge, M. Schmidtmann, and B. Tsukerblat, J. Cluster Sci. 16, 391-396 (2005); (g) A. Müller, M.T. Pope, A.M. Todea, H. Bögge, J. van Slageren, M. Dressel, P. Gouzerh, R. Thouvenot, B. Tsukerblat, and A. Bell, Angewante Chemie 119, 4561-4564 (2007).

[13] (a) J.M. Clemente-Juan, E. Coronado, and A. Gaita-Ariño, Chem. Soc. Rev. 41, 7464-7478 (2012). doi:10.1039/c2cs35205b; (b) E. Coronado, Nature Rev. Mater. 5, 87 (2020); (c) A. GaitaAriño, F. Luis, S. Hill, E. Coronado, Nature Chem. 11, 301 (2019).

[14] S. Cardona-Serra, J.M. Clemente Juan, A. Gaita-Ariño, N. Suaud, O. Svoboda, and E. Coronado, Chem. Commun. 49, 9621-9623 (2013). doi:10.1039/c3cc44859b

[15] S. Cardona-Serra, J.M. Clemente-Juan, E. Coronado, A. Gaita Ariño, N. Suaud, O. Svoboda, R. Bastardis, N. Guihéry, and J.J. Palacios, Chem. - Eur. J. 21, 763-769 (2015). doi:10.1002/chem.201404055

[16] L.-H. Bi, U. Kortz, M.H. Dickman, S. Nellutla, N.S. Dalal, B. Keita, L. Nadjo, M. Prinz, and R. Neumann, J. Cluster Sci. 17, 143-165 (2006). doi:10.1007/s10876-006-0048-y

[17] (a) A. Palii, S. Aldoshin, B. Tsukerblat, J.J. Borràs-Almenar, J.M. Clemente-Juan, S. CardonaSerra, and E. Coronado, Inorg. Chem. 56, 9547-9554 (2017); (b) A. Palii, S. Aldoshin, B. Tsukerblat, J.M. Clemente-Juan, A. Gaita-Ariño, and E. Coronado, Phys. Chem. Chem. Phys. 19, 26098-26106 (2017); (c) A. Palii, B. Tsukerblat, S. Klokishner, S. Aldoshin, D. Korchagin, and J.M. Clemente-Juan, J. Phys. Chem. C. 123, 2451-2459 (2019); (d) A. Palii, B. Tsukerblat, S. Klokishner, S. Aldoshin, D. Korchagin, and J.M. Clemente-Juan, J. Phys. Chem. C. 123, 2460-2473 (2019); (e) A. Palii, S. Aldoshin, B. Tsukerblat, J.M. Clemente-Juan, and E. Coronado, J. Phys. Chem. C. 123, 5746-5760 (2019); (f) A.V. Palii and B. Tsukerblat, J. Appl. Phys. 125 142901-142908 (2019); (g) A. Palii, S. Aldoshin, and B. Tsukerblat, J. Phys. Chem. C. 121, 27218-27224 (2017).

[18] M.N. Leuenberger and D. Loss, Nature. 410, 789-793 (2001). doi:10.1038/35071024

[19] F. Meier, L. Levy and D. Loss, Phys. Rev. B. 68, 134417-134432 (2003). doi:10.1103/PhysRevB. 68.134417

[20] (a) F. Troiani, A. Ghirri, M. Affronte, S. Carretta, P. Santini, G. Amoretti, S. Piligkos, G. Timco, and R.E.P. Winpenny, Phys. Rev. Lett. 94, 207208 (2005). (b) D.N. Woodruff, R.E.P. Winpenny, and R.A. Layfield, Chem. Rev. 113, 5110-5148 (2013).

[21] (a) M. Affronte, I. Casson, M. Evangelisti, A. Candini, S. Carretta, C.A. Muryn, S.J. Teat, G. Timco, G.W. Wernsdorfer, and R.E.P. Winpenny, Angew. Chem. Int. Ed. 44, 6496-6500 (2005); (b) G. Aromí, D. Aguilà, P.Gamez, F. Luis, and O. Roubeau, Chem. Soc. Rev. 41, 537-546 (2012); (c) M.A. AlDamen, S. Cardona-Serra, J.M. Clemente-Juan, E. Coronado, A. GaitaArino, C. Marti-Gastaldo, F. Luis, and O. Montero, Inorg. Chem. 48, 3467-3479 (2009); (d) Molecular Magnets: Physics and Applications, edited by J. Bartolomé, F. Luis, J.F. Fernández (Springer, 2016); (e) M.J. Martínez-Pérez, S. Cardona-Serra, C. Schlegel, F. Moro, P.J. Alonso, H. Prima-García, J.M. Clemente-Juan, M. Evangelisti, A. Gaita-Ariño, J. Sesé, J. Van Slageren, 
E. Coronado, and F. Luis, Phys. Rev. Let. 108, 247213 (2012); (f) F. Luis, F.L. Mettes, J. Tejada, D. Gatteschi, and L.J. De Jongh, Phys. Rev Lett. 85, 4377 (2000).

[22] (a) A. Ardavan, O. Rival, J.J.L. Morton, S.J. Blundell, A.M. Tyryshkin, G.A. Timco, and R.E.P. Winpenny, Phys. Rev. Lett. 98, 057201-057205 (2007); (b) ibid.; (c) C.J. Wedge, G.A. Timco, E.T. Spielberg, R.E. George, F. Tuna, S Rigby, E.J.L. McInnes, R.E.P. Winpenny, S.J. Blundell, A. Ardavan, Phys. Rev. Lett. 108 (10), 107204 (2012); (d) G.A. Timco, T.B. Faust, F. Tuna, and R.E.P. Winpenny, Chem. Soc. Rev. 40, 3067-3075 (2011); (e) W. Wernsdorfer, Nat. Mater. 6, 174-176 (2007).

[23] S. Carretta, P. Santini, G. Amoretti, F. Troiani, and M. Affronte, Phys. Rev. B. 76, 024408 (2007). doi:10.1103/PhysRevB.76.024408

[24] S.M. Aldoshin, E.B. Feldman, and M.A. Yurishchev, J. Exp. Theor. Phys. 107, 804-811 (2008). doi:10.1134/S1063776108110101

[25] G.A. Timco, S. Carretta, F. Troiani, F. Tuna, R.J. Pritchard, C.A. Muryn, E.J.L. McInnes, A. Ghirri, A. Candini, P. Santini, G. Amoretti, M. Affronte, and R.E.P. Winpenny, Nat. Nanotechnol. 4, 173-178 (2009). doi:10.1038/nnano.2008.404

[26] A. Candini, G. Lorusso, F. Troiani, A. Ghirri, S. Carretta, P. Santini, G. Amoretti, C. Muryn, F. Tuna, G. Timco, R.J.L. McInnes, E.J.L. McInnes, R.E.P. Winpenny, W. Wernsdorfer, and M. Affronte, Phys. Rev. Lett. 104, 037203 (2010). doi:10.1103/PhysRevLett.104.037203

[27] W. Wernsdorfer, Nat. Nanotechnol. 4, 145-146 (2009). doi:10.1038/nnano.2009.21

[28] L. Tesi, A.A. Lunghi, M. Atzori, E. Lucaccini, L. Sorace, F. Totti, and R. Sessoli, Dalton Trans. 45, 16635-16643 (2016). doi:10.1039/C6DT02559E

[29] J. Lehmann, A. Gaita-Ariño, E. Coronado, and D. Loss, Nat. Nanotech. 2, 312-317 (2007). doi:10.1038/nnano.2007.110

[30] D. Gatteschi and L. Pardi, Gazz. Chim. It. 123, 231 (1993).

[31] B.L. Silver, Irreducible Tensor Methods, an Introduction for Chemists (Academic Press, New York, 1976).

[32] D.A. Varshalovich, A.N. Moskalev, and V.K. Khersonskii, Quantum Theory of Angular Momentum (World Scientific, Singapore, 1988).

[33] E.P. Wigner, Group Theory and its Application to the Quantum Mechanics of Atomic Spectra (Academic Press, New York, 1959).

[34] B.S. Tsukerblat, Group Theory in Chemistry and Spectroscopy (Dover Pub. Inc, Mineola, New York, 2006).

[35] E. Coronado, R. Georges, and B.S. Tsukerblat, Exchange Interactions: Mechanisms, in: Localized and Itinerant Molecular Magnetism: From Molecular Assemblies to the Devices, NATO ASI Series, edited by E. Coronado, P. Delhaes, D. Gatteschi and J. Miller (Kluwer Acad. Publishers, Dordrecht, 1996), pp. 65-84.

[36] J.M. Clemente, R. Georges, A.V. Palii, and B.S. Tsukerblat, Exchange Interactions: Spin Hamiltonians, ibid., pp. 85-104.

[37] (a) R. Bǒca, Theoretical Foundations of Molecular Magnetism (Elsevier, Amsterdam, 1999); (b) D. Kouzoudis, J. Magn. Magn. Mater. 173, 259-273 (1997); (c) D. Kouzoudis, J. Magn. Magn. Mater. 189, 366-376 (1998).

[38] B.S. Tsukerblat and M.I. Belinsky, Magnetochemistry and Radiospectroscopy of Exchange Clusters (Pub. Stiintsa (Acad. Sci. Moldova), Kishinev, 1983 (Rus)).

[39] B.S. Tsukerblat, M.I. Belinskii, and V.E. Fainzilberg, Magnetochemistry and Spectroscopy of Transition Metal Exchange Clusters, in: Soviet Sci. Rev. B; M.E. Vol'pin, M.Ed. (Harwood Acad. Pub., New York, 1987), Vol. 9, pp. 337-481.

[40] J.J. Borras-Almenar, J.M. Clemente-Juan, E. Coronado, A.V. Palii, and B.S. Tsukerblat, Magnetic Properties of Mixed-Valence Systems: Theoretical Approaches and Applications, in: Magnetoscience-From Molecules to Materials, edited by J. Miller and M. Drillon (Willey-VCH, Weinheim, 2001), pp. 155-210.

[41] (a) J.J. Borras-Almenar, J.M. Clemente-Juan, E. Coronado, and B.S. Tsukerblat, Inorg. Chem. 38, 6081 (1999); (b) ibid., J. Comp. Chem. 22, 985 (2001).

[42] (a) J.J. Borras-Almenar, J.M. Clemente, E. Coronado, A.V. Palii, and B.S. Tsukerblat, J. Chem. Phys. 105, 6892-6909 (1996); (b) J.M. Clemente-Juan, J.J. Borras-Almenar, E. Coronado, 
A.V. Palii, and B.S. Tsukerblat, Inorg. Chem. 48, 4557-4568 (2009); (c) J.J. Borras-Almenar, S. Cardona-Serra, J.M. Clemente-Juan, E. Coronado, A.V. Palii, and B.S. Tsukerblat, J. Comp. Chem. 31, 1321-1332 (2010).

[43] (a) B. Tsukerblat, A. Palii, J.M. Clemente-Juan, and E. Coronado, A Symmetry Adapted Approach to the Dynamic Jahn-Teller Problem, in Series: Progress in Theoretical Chemistry and Physics, Vol. 23: Vibronic Interactions and the Jahn-Teller Effect (2011), pp. 39-58; (b) B. Tsukerblat, A. Palii, J.M. Clemente-Juan, A. Gaita-Ariño, and E. Coronado, Int. J. Quantum Chemistry 112, 2957 (2012); (c) J.M. Clemente-Juan, A. Palii, E. Coronado, and B. Tsukerblat, J. Chem. Theory Comp. 12, 3545-3560 (2016). (d) J.M. Clemente-Juan, A. Palii, B. Tsukerblat, and E. Coronado, J. Comp. Chem. 39, 1815-1827 (2018).

[44] (a) A. Müller and A.J. Döring, Angew. Chem. Int. Ed. Engl. 27, 1721 (1988); (b) D. Gatteschi, L. Pardi, A.-L. Barra, A. Müller, and J. Döring, Nature, 354, 465 (1991).

[45] (a) B. Tsukerblat, A. Tarantul, and A. Müller, Physics Letters A 353, 48 (2006); (b) B. Tsukerblat, A. Tarantul, and A. Müller, J. Chem. Phys. 125, 0547141 (2006); (c) A. Tarantul, B. Tsukerblat, and A. Müller, Chem. Phys. Let. 428, 361 (2006); (d) A. Tarantul, B. Tsukerblat, and A. Müller, Inorg. Chem. 46, 161 (2007); (e) A. Tarantul, B. Tsukerblat, and A. Müller, Solid State Sciences 10 (2008); 1814-1819; (f) B. Tsukerblat, A. Tarantul, and A. Müller, J. Mol. Structure, 838, 124 (2007); (g) B. Tsukerblat and A. Tarantul, The Nanoscopic V 15 Cluster: An Unique Magnetic Polyoxometalate, in Molecular Cluster Magnets, edited by R. Winpenny, Chapter 3 (World Sci. Pub., Singapore, 2011), pp. 106-180; (h) Z. Fu, Y. Xiao, Y. Su, Y. Zheng, P. Kögerler, and T. Brückel, EPL 112, 27003 (2015).

[46] (a) S. Bertaina, S. Gambarelli, T. Mitra, B. Tsukerblat, A. Müller, and B. Barbara, Nature, 453, 203-206 (2008); Nature, 466, 1006 (2010); (b) J.H. Shim, S. Bertaina, S. Gambarelli, T. Mitra, A. Müller, E.I. Baibekov, B. Z. Malkin, B. Tsukerblat, and B. Barbara, Phys. Rev. Lett. 109, 0504011-5 (2012); (c) A. Tarantul and B. Tsukerblat, Inorg. Chim. Acta 363, 4361-4367 (2010); (d) A. Tarantul and B. Tsukerblat, J. Phys. Conf. Series 324, 012007, 1-17 (2011).

[47] (a) N.F. Chilton, R.P. Anderson, L.D. Turner, A. Soncini, and K.S. Murray, J. Comput. Chem. 34, 1164-1175 (2013); (b) N.F. Chilton, D. Collison, E.J.L. McInnes, R.E.P. Winpenny, and A. Soncini, Nat. Commun. 4, 2551 (2013), doi:10.1038/ncomms3551.

[48] (a) R. Schnalle and J. Schnack, Phys. Rev. B 79, $104419-9$ (2009); (b) R. Schnalle, A.M. Läuchli, and J. Schnack, Cond. Mat. Phys. 12, 331 (2009); (c) O. Waldmann, Phys. Rev. B. 61, 6138-6144 (2000).

[49] (a) Schnack, Dalton Trans. 39, 4677-4686 (2010); (b) M.L. Baker, G.A. Timco, S. Piligkos, J.S. Mathieson, H. Mutka, F. Tuna, P. Kozlowski, M. Antkowiak, T. Guidi, T. Gupta, H. Rath, R.J. Woolfson, G. Kamieniarz, R.G. Pritchard, H. Weihe, L. Cronin, G. Rajaraman, D. Collison, E.J.L. McInnes, and R.E.P. Winpenny, PNAS 109, 19113-19118 (2012); (c) G. Kamieniarz, W. Florek, and M. Antkowiak, Phys. Rev. B. 92, 140411 (2015).

[50] G.F. Koster, J.O. Dimmok, R.G. Wheeler, and H. Statz, Properties of the Thirty-Two Point Groups (M.I.T. Press, Cambridge, Mass., 1963).

[51] (a) R. Englman, The Jahn-Teller Effect in Molecules and Crystals (Wiley, London, 1972); (b) I.B. Bersuker and V.Z. Polinger, Vibronic Interactions in Molecules and Crystals (Springer-Verlag, Berlin, 1989); (c) I.B. Bersuker, The Jahn-Teller Effect (Cambridge University Press, 2006).

[52] E. Dagotto, J. Riera, and D. Scalapino, Phys. Rev. B. 45, 5744 (1992). doi:10.1103/PhysRevB.45. 5744

[53] D. Poilblanc, H. Tsunetsugu, and T.M. Rice, Phys. Rev. B. 50, 6511 (1994). doi:10.1103/Phys RevB.50.6511

[54] E. Rentschler, D. Gatteschi, A. Cornia, A.C. Fabretti, A.L. Barra, O.I. Shchegolikhina, and A.A. Zhdanov, Inorg. Chem. 35, 4427 (1996). doi:10.1021/ic951317g

[55] A. Lascialfari, D. Gatteschi, F. Borsa, and A. Cornia, Phys. Rev. B. 55, 14341 (1997). doi:10.1103/PhysRevB.55.14341

[56] A. Caneschi, A. Cornia, A.C. Fabretti, and D. Gatteschi, Angew. Chem. Int. Ed. Engl. 34, 2716 (1995). doi:10.1002/anie.199527161

[57] A. Caneschi, A. Cornia, and S.J. Lippard, Angew. Chem. Int. Ed. Engl. 34, 467 (1995). doi:10.1002/anie.199504671 
[58] (a) T. Yamase, K. Fukaya, H. Nojiri, and Y. Ohshima, Inorg. Chem. 45, 7698 (2006); (b) N. Zamstein, A. Tarantul, and B. Tsukerblat, Inorg. Chem. 46, 8851 (2007).

[59] (a) J. Schnack and M. Luban, Phys. Rev. B. 63, 014418 (2001); (b) O. Waldmann, Phys. Rev. B. 65, 024424 (2001); (c) J. Schnack, M. Luban, and R. Modler, Europhys. Lett. 56, 863 (2001); (d) O. Waldmann, Phys. Rev. B. 75, 012415-012419 (2007).

[60] (a) D. Gatteschi, R. Sessoli, W. Plass, A. Müller, E. Krickemeyer, J. Meyer, D. Sölter, and P. Adler, Inorg. Chem., 35, 1926 (1995); (b) B. Tsukerblat, Inorg. Chimica Acta 361, 3746-3760 (2008);

(c) P. Kögerler, B. Tsukerblat, A. Müller, Dalton Trans. 39, 21-36 (2010).

[61] (a) C. Zener, Phys. Rev. 82, 403 (1951); (b) P.W. Anderson and H. Hasegawa, Phys. Rev. 100, 675 (1955).

[62] S. Drüeke, P. Chaudhuri, K. Pohl, K. Wieghardt, X.Q. Ding, E. Bill, A. Sawaryn, A.X. Trautwein, H. Winkler, and S.J. Gurman, J. Chem. Soc. Chem. Commun. 59, 59-62 (1989).

[63] X.Q. Ding, E.L. Bominaar, B.E. Winkler, A.X.H. Trautwein, S. Drüeke, P. Chaudhuri, and K. Wieghardt, J. Chem. Phys. 92, 178 (1990). doi:10.1063/1.458460

[64] (a) M.I. Belinsky, Inorg. Chem. 47, 3521 (2008); (b) M.I. Belinskii, Mol. Phys. 60, 793 (1987); (c) M.I. Belinskii, Chem. Phys. 173, 27 (1993); (d) M.I. Belinskii, Chem. Phys. 189, 451 (1994).

[65] (a) Mixed Valence Compounds, edited by D.B. Brown (Riedel Publ. Comp., Dordrecht, 1979); (b) Mixed Valence Systems: Applications in Chemistry, Physics and Biology, edited by K. Prassides (Kluwer Academic Publishers, Dordrecht, 1991), Vol. 343; (c) G. Blondin and J.J. Girerd, Chem. Rev. 90, 1359 (1989).

[66] C.J. Calzado, J.M. Clemente-Juan, E. Coronado, A. Gaita-Arino, and N. Suaud, Inorg. Chem. 47, 5889 (2008). doi:10.1021/ic8002355

[67] A. Müller, R. Sessoli, E. Krickemeyer, H. Bögge, J. Meyer, D. Gatteschi, L. Pardi, J. Westphal, K. Hovemeier, R. Rohlfing, J. Döring, F. Hellweg, C. Beugholt, and M. Schmidtmann, Inorg. Chem. 36, 5239 (1997). doi:10.1021/ic9703641

[68] (a) J.J. Borras-Almenar, J.M. Clemente-Juan, E. Coronado, and B.S. Tsukerblat, Chem. Phys. 195, 1 (1995); (b) J.J. Borras-Almenar, J.M. Clemente-Juan, E. Coronado, and B.S. Tsukerblat, Chem. Phys. 195, 17 (1995); (c) J.J. Borras-Almenar, J.M. Clemente-Juan, E. Coronado, and B.S. Tsukerblat, Chem. Phys. 195, 29 (1995).

[69] H. Goff and G.N. La Mar, J. Am. Chem. Soc. 99, 6599 (1977). doi:10.1021/ja00462a022

[70] (a) N. Suaud, A. Gaita-Ariño, J.M. Clemente-Juan, J. Sanchez.Martín, and E. Coronado, J. Am. Chem. Soc. 124, 15134-15140 (2004); (b) N. Suaud, A. Gaita-Ariño, J.M. Clemente-Juan, and E. Coronado, Chem. Eur. J. 10, 4041-4053 (2004).

[71] (a) K.Y. Wong and P.N. Schatz, Prog. Inorg. Chem. 28, 369 (1981); (b) S.B. Piepho, J. Am. Chem. Soc. 110, 6319 (1988); (c) S.B. Piepho, J. Am. Chem. Soc. 112, 4197 (1990); (c) S.B. Piepho, E.R. Krausz, and P.N. Schatz, J. Am. Chem. Soc. 100, 2996 (1978).

[72] B.S. Brunschwig, C. Creutz, and N. Sutin, Chem. Soc. Rev. 31, 168 (2002). doi:10.1039/b008034i

[73] K. Takatsuka, T. Yonehara, and K. Hanasaki, Chemical Theory Beyond the Born-Oppenheimer Paradigm: Nonadiabatic Electronic and Nuclear Dynamics in Chemical Reactions (World Scientific Publishing Co. Pte. Ltd., Singapore, 2015).

[74] (a) A.-L. Barra, D. Gatteschi, B.S. Tsukerblat, J. Döring, A. Müller, and L.-C. Brunel, Inorg. Chem. 31, 5132 (1992); (b) D. Gatteschi and B.S. Tsukerblat, Chem. Phys. 202, 25 (1996); (c) D. Gatteschi, B.S. Tsukerblat, and V.E. Fainzilberg, Appl. Magnet. Reson. 10, 217 (1996).

[75] (a) D. Gatteschi, B.S. Tsukerblat, A.L. Barra, L.C. Brunel, A. Müller, and J. Döring, Inorg. Chem. 32, 2114 (1993); (b) D. Gatteschi and B.S. Tsukerblat, Mol. Phys. 79, 121 (1993).

[76] (a) B. Tsukerblat, A. Palii, J.M. Clemente-Juan, and E. Coronado, J. Phys. Conf. Series 833, 012002 (2017); (b) B. Tsukerblat, A. Palii, J.M. Clemente-Juan, N. Suaud, and E. Coronado, Acta Phys. Polonica A. 133, 329 (2018).

[77] (a) A. Palii, B. Tsukerblat, J.M. Clemente-Juan, and E. Coronado, Int. Revs Phys. Chem. 29, 135-230 (2010); (b) J.J. Borrás-Almenar, J.M. Clemente-Juan, E. Coronado, A.V. Palii, and B.S. Tsukerblat, Chem. Phys. 274, 131 (2001); (c) J.J. Borrás-Almenar, J.M. Clemente-Juan, E. Coronado, A.V. Palii, and B.S. Tsukerblat, Chem. Phys. 274, 145 (2001); (d) J.J. Borrás-Almenar, J.M. Clemente-Juan, E. Coronado, A.V. Palii, and B.S. Tsukerblat, J. Chem. Phys. 114, 1148 (2001); 
(e) J.J. Borrás-Almenar, J.M. Clemente-Juan, E. Coronado, A.V. Palii, and B.S. Tsukerblat, J. Sol. State. Chem. 159, 268-280 (2001).

[78] B.S. Tsukerblat, A.V. Palii, V.Y. Mirovitskii, S.M. Ostrovsky, K. Turta, T. Jovmir, S. Shova, J. Bartolome, M. Evangelisti, and G. Filoti, J. Chem. Phys. 115, 9528-9535 (2001). doi:10.1063/1.1413988

[79] C.P. Berlinguette, D. Vaughn, C. Cañada-Vilalta, J.-R. Galán-Mascarós, and K.R. Dunbar, Angew. Chem. Int. Ed. 42, 1523-1526 (2003). doi:10.1002/anie.200250479

[80] A.V. Palii, S.M. Ostrovsky, S.I. Klokishner, B.S. Tsukerblat, C.P. Berlinguette, K.R. Dunbar, and J.R. Galán-Mascarós, J. Am. Chem. Soc. 126, 16860-16867 (2004). doi:10.1021/ja046771a

[81] (a) J.J. Novoa, M. Deumal, and J. Jornet-Somoza, Chem. Soc. Rev. 40, 3182-3212 (2011); (b) J.P. Malrieu, R. Caballol, C.J. Calzado, C. de Graaf, and N. Guihéry, Chem. Rev. 114, 429-492 (2014).

[82] (a) D. Reinen, M. Atanasov, P. Kohler, and D. Babel, Coord. Chem. Rev. 254, 2703-2754 (2010); (b) M. Atanasov, J.M. Zadrozny, J.R. Long, and F. Neese, Chem. Sci. 4, 139-156 (2013). 